Uma versão parametrizada do teorema de Borsuk-Ulam 
SERVIÇO DE PÓS-GRADUAÇÃO DO ICMC-USP

Data de Depósito:

Assinatura:

\section{Uma versão parametrizada do teorema de Borsuk-Ulam}

\section{Nelson Antonio Silva}

Orientadora: Profa. Dra. Denise de Mattos

Dissertação apresentada ao Instituto de Ciências Matemáticas e de Computação - ICMC-USP, como parte dos requisitos para obtenção do título de Mestre em Ciências - Matemática. VERSÃO REVISADA.

USP - São Carlos

Abril/2011 
Ficha catalográfica elaborada pela Biblioteca Prof. Achille Bassi e Seção Técnica de Informática, ICMC/USP, com os dados fornecidos pelo(a) autor(a)

Silva, Nelson Antonio
Uma versão parametrizada do teorema de Borsuk-
Ulam / Nelson Antonio Silva; orientadora Denise de
Mattos -- São Carlos, 2011.
87 p.
Dissertação (Mestrado - Programa de Pós-Graduação en
Matemática) -- Instituto de Ciências Matemáticas e
de Computação, Universidade de São Paulo, 2011.
1. Teorema de Borsuk-Ulam. 2. Fibrados. 3.
Cohomologia de Cech. 4. Classes características. 5.
Teorema de Leray-Hirsch. I. de Mattos, Denise,
orient. II. Título.


Aos meus pais Luiz e Wilma.

$E$ aos meus irmãos

Didio e Carin. 



\section{Agradecimentos}

Em primeiro lugar, agradeço aos meus pais por seus ensinamentos, valores e conselhos, que nunca, em nenhuma instituição, poderia obtê-los. Por me apoiarem em todas as minhas escolhas, estarem presentes em todas as etapas da minha vida e por terem me dado o melhor exemplo de amor e carinho.

Aos meus irmãos, por serem meus amigos e pessoas nas quais sempre irei me espelhar.

Aos meus amigos, pelo companheirismo, pelas conversas e por deixarem essa etapa mais prazerosa.

À minha orientadora Denise, por ter acreditado em mim, por seu conhecimento, pela atenção, dedicação, preocupação e por estar sempre disponível a me ajudar, mesmo em período de férias, com valiosos conselhos. Ao professor Edivaldo, que assim como a Denise, esteve sempre presente, me dando importantes sugestões, sanando minhas dúvidas e me incentivando. Muito obrigado por tudo!

À todos os professores e funcionários do ICMC, em especial, a minha orientadora de graduação, Ires Dias, muito obrigado.

À Fapesp pelo apoio financeiro para realização deste trabalho. 



\section{Resumo}

O teorema clássico de Borsuk-Ulam nos dá informações à respeito de aplicações $S^{n} \longrightarrow \mathbb{R}^{n}$, no qual $S^{n}$ é um $\mathbb{Z}_{2}$-espaço livre. O teorema afirma que existe pelo menos uma órbita que é enviada em um único ponto em $\mathbb{R}^{n}$.

Dold [9] estendeu este problema para o contexto de fibrados, considerando aplicações $f$ : $S(E) \longrightarrow E^{\prime}$ nos quais preservam fibras; aqui, $S(E)$ denota o espaço total do fibrado em esfera sobre $B$ associado ao fibrado vetorial $E \longrightarrow B$ e $E^{\prime} \longrightarrow B$ é outro fibrado vetorial. O objetivo desse trabalho é provar esta versão do teorema de Borsuk-Ulam obtida por Dold, chamada versão parametrizada do teorema de Borsuk-Ulam. Nós também provamos uma versão cohomológica deste problema. 



\section{Abstract}

The classical Borsuk-Ulam Theorem gives information about maps $S^{n} \longrightarrow \mathbb{R}^{n}$ where $S^{n}$ has a free action of the cyclic group $\mathbb{Z}_{2}$. The theorem states that there is at least one orbit which is sent to a single point in $\mathbb{R}^{n}$.

Dold [9] extended this problem to a fibre-wise setting, by considering maps $f: S(E) \longrightarrow E^{\prime}$ which preserve fibres; here, $S(E)$ denotes the total space of the sphere bundle associated over $B$ to a vector bundle $E \longrightarrow B$ and $E^{\prime} \longrightarrow B$ is other vector bundle over $B$. The purpose of this work is to prove this version of the Borsuk-Ulam theorem obtained by A. Dold, called parametrized version of the Borsuk-Ulam theorem. We also prove a cohomological generalization of this problem. 



\section{Sumário}

Introdução

1 Fibrados Vetoriais 1

1.1 A categoria VB . . . . . . . . . . . . . . . . . . 1

1.2 A Variedade de Grassmann . . . . . . . . . . . . . . . . . . . . 4

1.3 Soma de Whitney e Fibrados Induzidos $\ldots \ldots \ldots$

1.4 Aplicação de Gauss $\ldots \ldots \ldots \ldots$. . . . . . . . . . . . . . 8

1.5 Métrica em um fibrado vetorial . . . . . . . . . . . . . . . . . . . . 10

1.6 Fibrados associados $\ldots \ldots \ldots \ldots$. . . . . . . . . . . . . . . . 12

1.7 Fibrado Vetorial Universal $\ldots \ldots \ldots$. . . . . . . . . . . . . . . . . 13

$1.8 \quad$ Kernel, Imagem e Cokernel $\ldots \ldots \ldots \ldots \ldots$

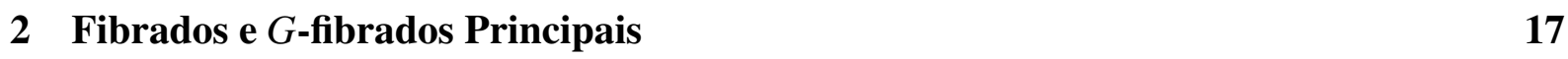

$2.1 \quad$ Grupos Topológicos . . . . . . . . . . . . . . . . . . . . . . 17

2.2 A categoria $\mathbf{P B} \ldots \ldots \ldots \ldots \ldots \ldots$

2.3 Fibrados . . . . . . . . . . . . . . . . . . . . . 23

2.4 Fibrados Coordenados $\ldots \ldots \ldots \ldots \ldots$

\begin{tabular}{lll}
\hline 3 & Cohomologia & 27
\end{tabular}

3.1 Sistemas Diretos $\ldots \ldots \ldots \ldots \ldots$. . . . . . . . . . . . . . . . 27

3.2 Limites Diretos $\ldots \ldots \ldots \ldots$. . . . . . . . . . . . . . . . . . . 29

3.3 A cohomologia simplicial $\ldots \ldots \ldots \ldots \ldots \ldots \ldots$ 
$3.3 .1 \quad$ Os pares simpliciais $\ldots \ldots \ldots \ldots \ldots \ldots \ldots \ldots$

3.3 .2 Os $R$-módulos de Cohomologia Simplicial Relativos . . . . . . . . . . 33

3.4 O nervo de uma cobertura . . . . . . . . . . . . . . . 35

3.5 A cohomologia de Čech . . . . . . . . . . . . . . . . . 38

3.5.1 Os $R$-módulos de cohomologia de Čech . . . . . . . . . . . . . . 38

3.5.2 Os axiomas de Eilenberg-Steenrod . . . . . . . . . . . . . . . . . 39

$3.5 .3 \quad$ o produto cup $\cup \ldots \ldots \ldots \ldots$

4 Classes Características 45

$4.1 \quad$ Fórmulas de Künneth $\ldots \ldots \ldots$. . . . . . . . . . . . . . . . . . . . . . . 45

$4.2 \quad \mathrm{O}$ teorema de Leray-Hirsch $\ldots \ldots \ldots \ldots \ldots$. . . . . . . . . . . 48

4.3 As classes de Stiefel-Whitney $\ldots \ldots \ldots$. . . . . . . . . . . . 50

4.4 Unicidade das classes de Stiefel-Whitney $\ldots \ldots \ldots$. . . . . . . . . . . . 55

4.5 A classe característica de Euler . . . . . . . . . . . . . . . . . . 56

5 Versão parametrizada do teorema de Borsuk-Ulam 59

5.1 Versão parametrizada do teorema de Borsuk-Ulam para fibrados em esfera . . . 60

5.2 Versão Cohomológica . . . . . . . . . . . . . . . . . . . . . 63

$\begin{array}{lll}6 & \text { Apêndice } & 69\end{array}$

6.1 Produto Tensorial . . . . . . . . . . . . . . . . . . . . . . 69

6.2 Sistemas Inversos $\ldots \ldots \ldots \ldots \ldots \ldots$

6.3 Limites Inversos $\ldots \ldots \ldots \ldots \ldots$

6.4 Cohomologia de Alexander . . . . . . . . . . . . . . . 73

6.4 .1 Sequência de Mayer-Vietoris e o produto cup . . . . . . . . . . . . . . 74

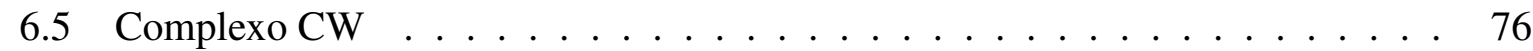

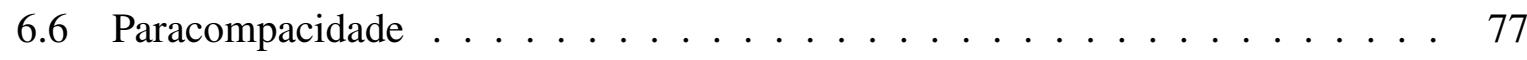

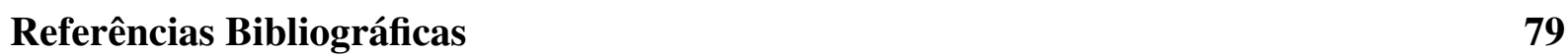

\begin{tabular}{ll}
\hline Índice Remissivo & 82
\end{tabular} 


\section{Introdução}

O teorema clássico de Borsuk-Ulam afirma que toda função contínua $f: S^{n} \longrightarrow \mathbb{R}^{n}$ colapsa pelo menos um par de pontos antípodas, isto é, existem $x,-x \in S^{n}$ tais que $f(x)=f(-x)$.

Esse famoso teorema foi conjecturado por Stanislaw Ulam e provado, em 1933, por Karol Borsuk [1]. Desde então, inúmeras versões deste resultado têm sido publicadas. São muitas as técnicas utilizadas na demonstração de cada uma destas versões.

O presente texto tem por objetivo apresentar uma demonstração de uma versão desse teorema conhecida como Versão parametrizada do teorema de Borsuk-Ulam, provada por A. Dold em [9]. Esta é uma versão do teorema no contexto de fibrados. Considerando dois fibrados vetoriais $\xi=\left(E, \pi, B, \mathbb{R}^{m}\right)$ e $\xi^{\prime}=\left(E^{\prime}, \pi^{\prime}, B, \mathbb{R}^{n}\right)$ com mesmo espaço base $B$ paracompacto e uma aplicação $\mathbb{Z}_{2}$-equivariante $f: S(E) \longrightarrow E^{\prime}$ que preserva fibras (isto é, $\pi^{\prime} \circ f=\left.\pi\right|_{S(E)}$ ), no qual $S(E)$ denota o espaço total do fibrado em esfera associado de $\xi$, a versão parametrizada do teorema de Borsuk-Ulam consiste em estimar a dimensão cohomológica do conjunto

$$
\overline{Z_{f}}=\{[x] \in \overline{S(E)} ; \bar{f}([x])=0\}
$$

no qual $\overline{S(E)}$ é o conjunto de órbitas de $S(E)$ pela ação antipodal de $\mathbb{Z}_{2}$ e $\bar{f}$ é a aplicação induzida por esta ação. Mais especificamente,

$$
\operatorname{dim}_{\mathrm{c}} \overline{Z_{f}} \geq \operatorname{dim}_{\mathrm{c}} B+m-n-1,
$$

no qual $\operatorname{dim}_{\mathrm{c}}$ significa dimensão cohomológica.

A técnica empregada em sua demonstração é a técnica dos polinômios característicos. O 
primeiro passo será calcularmos o anel de cohomologia $\check{H}^{*}(\overline{S(E)})$, no qual a notação $\check{H}$ significa que estamos trabalhando com os módulos de cohomologia de Čech. Este anel pode ser calculado utilizando como ferramenta o Teorema de Leray-Hirsch e é descrito em termos de um polinômio especial, chamado polinômio de Stiefel-Whitney de $\xi$, definido como

$$
\omega(E, t)=\sum_{j=0}^{m} \omega_{j}(E) t^{m-j} \in \check{H}^{*}(B)[t],
$$

no qual as classes $\omega_{j}(E)$ são as classes de Stiefel-Whitney de $\xi$. Mais especificamente,

$$
\check{H}^{*}(\overline{S(E)}) \cong \frac{\check{H}^{*}(B)[t]}{(\omega(E, t))}
$$

No caso particular em que $B$ é constituído de um único ponto e a dimensão de $\xi$ é igual a $n+1$, temos o teorema em sua versão clássica.

Outras versões parametrizadas do Teorema de Borsuk-Ulam são encontradas nos trabalhos de: Nakaoka [31], Izydorek e Rybicki [22], Jaworowski [21], Volovikov [36], Koikara e Mukerjee [24] e De Mattos e Dos Santos [5].

Esse trabalho está organizado como segue. O capítulo 1 introduz a teoria básica de fibrados vetoriais. Na Seção 1.2 é apresentado um dos exemplos mais importantes de fibrado vetorial: o fibrado vetorial canônico e sua importância é vista na Seção 1.7 . A Seção 1.3 dedica-se à exposição de algumas maneiras de se obter novos fibrados vetoriais através de outros já conhecidos. O fibrado em esfera, juntamente com o fibrado projetivo associado a um fibrado vetorial, são definidos na Seção 1.6. A construção de tais fibrados utiliza o conceito de métrica, apresentado na Seção 1.4. Finalmente, na Seção 1.8 , é definida uma sequência exata para fibrados vetoriais.

$\mathrm{O}$ capítulo 2 destina-se à definição dos conceitos de $G$-fibrados principais e fibrados. A definição de fibrado será dada como em Husemoller [19], que diferentemente de Steenrod [35], define primeiramente $G$-fibrados principais e, posteriormente, fibrados associados. A Seção 2.1 apresenta resultados elementares da teoria de grupos topológicos, essenciais para o restante do capítulo. Na Seção 2.2 é definido o conceito de $G$-fibrado principal, no qual $G$ é um grupo topológico. A Seção 2.3 trata da definição de fibrado associado a um $G$-fibrado principal. Na Seção 2.4 são apresentados o conceito de fibrado coordenado e uma maneira de se obter fibrados 
através de funções conhecidas como funções transições. Também é vista a relação entre fibrados e fibrados vetoriais.

No capítulo 3 apresentamos a construção dos $R$-módulos de cohomologia de Čech relativos, no qual $R$ é um anel comutativo com unidade. Para tanto, é necessária a descrição de alguns conceitos básicos tais como sistemas diretos, limite direto de $R$-módulos, os $R$-módulos de cohomologia simplicial relativo e o nervo de uma cobertura, como veremos nas seções 3.1, 3.2. 3.3 e 3.4 , respectivamente. Na Seção 3.5 definimos os $R$-módulos de cohomologia de Čech. Usando o isomorfismo entre a cohomologia de Alexander e a cohomologia de Čech, definimos a sequência de Mayer-Vietoris e produto cup com abertos para a cohomologia de Čech.

O capítulo 4 dedica-se à exposição das classes características de Stiefel-Whitney de um fibrado vetorial (Seção 4.3) e da classe característica de Euler de um par fibrado (Seção 4.5). Para isto, são utilizados resultados importantes como a fórmula de Künneth (Seção 4.1), o teorema de Leray-Hirsch (Seção 4.2) e o teorema do isomorfismo de Thom.

O capítulo 5 contém o resultado central desse trabalho, que é a demonstração detalhada da Versão parametrizada do teorema de Borsuk-Ulam provada por Dold.

Finalmente, o capítulo 6 traz os conceitos e resultados elementares necessários para uma complementação da leitura dos capítulos anteriores. 


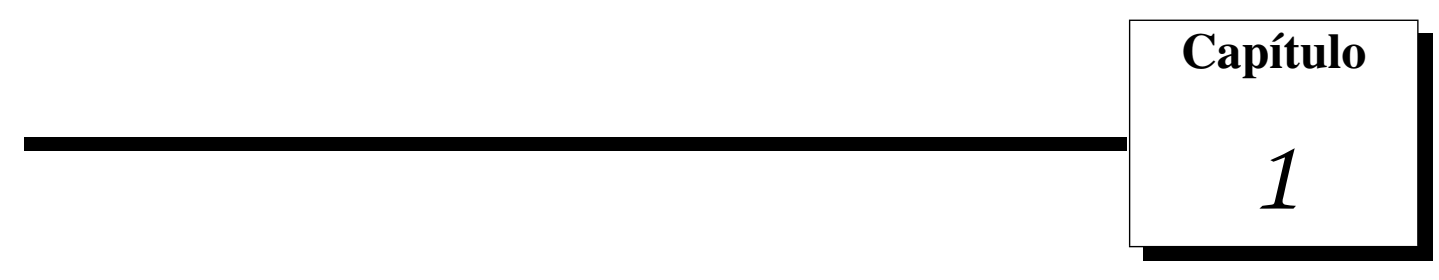

\section{Fibrados Vetoriais}

Esse capítulo introduz a teoria básica de Fibrados Vetoriais. Na Seção 1.1 é apresentada a definição de fibrado vetorial, que pode ser descrito como uma função contínua definida sobre uma coleção de espaços vetoriais, chamados fibras, em um espaço topológico, chamado espaço base. Estas fibras estão coladas por uma topologia, formando um espaço topológico, chamado espaço total, que localmente se comporta como um produto cartesiano. Na Seção 1.2 é apresentado um dos exemplos mais importantes de fibrado vetorial: o fibrado vetorial canônico e sua importância é vista na Seção 1.7. A Seção 1.3 dedica-se à exposição de algumas maneiras de se obter novos fibrados vetoriais através de outros já conhecidos. O fibrado em esfera, juntamente com o fibrado projetivo associado a um fibrado vetorial, são definidos na Seção 1.6 . A construção de tais fibrados utiliza o conceito de métrica, apresentado na Seção 1.4 . Finalmente, na Seção 1.8, é definida uma sequência exata para fibrados vetoriais. As referências básicas para este capítulo são: Fibre Bundles de D. Husemoller [19], Vector Bundles and K-Theory de A. Hatcher [17] e Characteristic Classes de J.W. Milnor e J.D.Stasheff [28].

\subsection{A categoria VB}

Definição 1.1.1. Um fibrado vetorial real $n$-dimensional com base $B$ é uma quádrupla $\xi=$ $\left(E, \pi, B, \mathbb{R}^{n}\right)$, no qual $\pi: E \longrightarrow B$ é uma função contínua entre espaços topológicos que satisfaz as seguintes condições: 
1. Para cada $b \in B$, o espaço $\pi^{-1}(b)$ possui uma estrutura de $\mathbb{R}$-espaço vetorial n-dimensional;

2. Condição de Trivialidade local: Para cada $b \in B$, existem uma vizinhança aberta $U \subset B$ de b e um homeomorfismo

$$
h: U \times \mathbb{R}^{n} \longrightarrow \pi^{-1}(U)
$$

tal que para todo $b^{\prime} \in U,\left.h\right|_{\left\{b^{\prime}\right\} \times \mathbb{R}^{n}}:\left\{b^{\prime}\right\} \times \mathbb{R}^{n} \longrightarrow \pi^{-1}\left(b^{\prime}\right)$ é um isomorfismo entre espaços vetoriais e $\left.\pi\right|_{\pi^{-1}(U)} \circ h=\pi_{1}$ (projeção na primeira coordenada de $U \times \mathbb{R}^{n}$ em $U$ ).

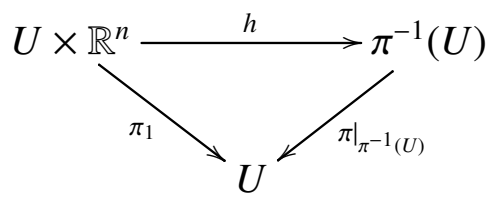

O espaço $E$ é chamado espaço total e $B$ é chamado espaço base. A função $\pi$ é chamada projeção do fibrado vetorial $\xi$ e, para cada $b \in B$, o espaço $\pi^{-1}(b)$ é chamado fibra sobre $b$. A coleção dos pares $(U, h)$ é chamada atlas do fibrado vetorial $\xi$ e o homeomorfismo $h$ é chamado carta local.

Em muitas ocasiões, um fibrado vetorial será denotado apenas por $\xi^{n}$, no qual $n \in \mathbb{N}$ indica a dimensão do fibrado vetorial. Neste caso, o espaço total, o espaço base e a projeção serão denotados por $E(\xi), B(\xi)$ e $\pi_{\xi}$, respectivamente.

Exemplo 1.1.2 (Fibrado vetorial trivial).

Seja $B$ um espaço topológico. Então, $\theta^{n}=\left(B \times \mathbb{R}^{n}, \pi_{1}, B, \mathbb{R}^{n}\right)$ é um fibrado vetorial de dimensão $n$, no qual $\left\{B, I d_{B}\right\}$ é o atlas deste fibrado e $I d_{B}: B \longrightarrow B$ é a função identidade.

Definição 1.1.3. Seja $\xi=\left(E, \pi, B, \mathbb{R}^{n}\right)$ um fibrado vetorial com base $B$. Um subfibrado vetorial de $\xi k$-dimensional, com $k<n$, é um fibrado vetorial $\eta=\left(E^{\prime}, \pi^{\prime}, B, \mathbb{R}^{k}\right)$, no qual $E^{\prime} \subset E$ e $\pi^{\prime}=\left.\pi\right|_{E^{\prime}}: E^{\prime} \rightarrow$ B é tal que $\pi^{-1}(b)$ é um subespaço vetorial $k$-dimensional de $\pi^{-1}(b)$.

Exemplos 1.1.4. Considere o fibrado vetorial trivial $\theta=\left(S^{n} \times \mathbb{R}^{n+1}, \pi_{1}, S^{n}, \mathbb{R}^{n+1}\right)$ e os conjuntos $T=\left\{(v, x) \in S^{n} \times \mathbb{R}^{n+1} ;\langle v, x\rangle=0\right\}$ e $N=\left\{(v, x) \in S^{n} \times \mathbb{R}^{n+1} ; x=\lambda v\right.$, com $\left.\lambda \in \mathbb{R}\right\}$. Então, $\tau\left(S^{n}\right)=\left(T,\left.\pi_{1}\right|_{T}, S^{n}, \mathbb{R}^{n}\right)$ é um subfibrado vetorial de $\theta$, no qual sua fibra é um subespaço vetorial de $\mathbb{R}^{n+1}$ de dimensão $n$, chamado fibrado vetorial tangente de $S^{n}$. Também, 
$\eta\left(S^{n}\right)=\left(N,\left.\pi_{1}\right|_{N}, S^{n}, \mathbb{R}\right)$ é um subfibrado vetorial de $\theta$, chamado fibrado vetorial nomal de $S^{n}$, no qual sua fibra é um subespaço vetorial de $\mathbb{R}^{n+1}$ de dimensão 1 .

Definição 1.1.5. Sejam $\xi=\left(E, \pi, B, \mathbb{R}^{n}\right)$ e $\eta=\left(E^{\prime}, \pi^{\prime}, B^{\prime}, \mathbb{R}^{m}\right)$ dois fibrados vetoriais. Um morfismo entre $\xi$ e $\eta$ é um par de funções contínuas $\left(u: E \longrightarrow E^{\prime}, f: B \longrightarrow B^{\prime}\right)$ tal que $\pi^{\prime} \circ u=f \circ \pi$, ou seja, o diagrama

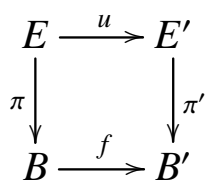

é comutativo e, além disso, $\left.u\right|_{\pi^{-1}(b)}: \pi^{-1}(b) \longrightarrow \pi^{-1}(f(b))$ é uma transformação linear. Este morfismo é denotado por $(u, f): \xi \longrightarrow \eta$. No caso em que $B=B^{\prime}$ e $f=I d_{B}$, o morfismo $\left(u, I d_{B}\right)$, ou simplesmente $u$, é chamado um B-morfismo .

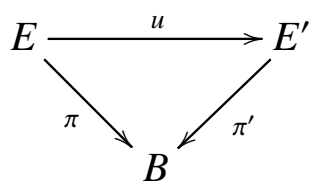

É possível verificar que dados três fibrados vetoriais $\xi, \eta$ e $\delta$ e dois morfismos $(u, f): \xi \longrightarrow \eta$ e $(v, g): \eta \longrightarrow \delta$, as composições $v \circ u: E(\xi) \longrightarrow E(\delta)$ e $g \circ f: B(\xi) \longrightarrow B(\delta)$ formam um morfismo $(v \circ u, g \circ f): \xi \longrightarrow \delta$. Também, se $\xi=\eta, u=I d_{E(\xi)}$ e $f=I d_{B(\xi)}$, então $(v \circ u, g \circ f)=(v, g)$. A associatividade entre os morfismos de fibrados vetoriais segue da associatividade de funções contínuas. Assim, os fibrados vetoriais e seus morfismos formam uma categoria.

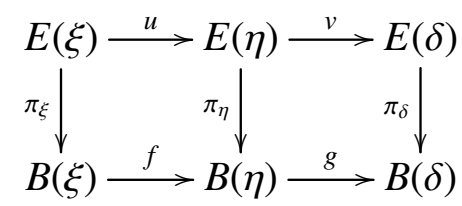

Definição 1.1.6. A categoria VB dos fibrados vetoriais é a categoria cujos objetos são os fibrados vetoriais e os morfimos são os morfismos entre fibrados vetoriais. A categoria VB possui como subcategorias, as categorias $\mathbf{V B}_{\mathbf{B}}$ dos fibrados vetoriais com base B e B-morfismos, $\mathbf{V B}^{\mathbf{n}}$ dos fibrados vetoriais de dimensão ne morfismos entre fibrados vetoriais $e \mathbf{V B}_{\mathbf{B}}^{\mathbf{n}}=\mathbf{V B}_{\mathbf{B}} \cap \mathbf{V B}^{\mathbf{n}}$ dos fibrados vetoriais $n$-dimensionais com base B e B-morfismos.

A notação VB é originada do termo em inglês vector bundle, que significa fibrado vetorial. 
Definição 1.1.7. Sejam $\xi$ e $\eta$ dois fibrados vetoriais em $\mathbf{V B}_{B}^{n}$, isto é, dois fibrados vetoriais com mesmo espaço base B e mesma dimensão $n$. Então, $\xi$ é B-isomorfo a $\eta$ quando existem dois $B$ morfismos $u: \xi \longrightarrow \eta e v: \eta \longrightarrow \xi$ tal que $(u \circ v)=\left(\operatorname{Id}_{E(\eta)}\right): \eta \longrightarrow \eta e(v \circ u)=\left(\operatorname{Id}_{E(\xi)}\right): \xi \longrightarrow \xi$.

O teorema a seguir é um critério para verificar se um dado $B$-morfismo é um $B$-isomorfismo.

Proposição 1.1.8. Sejam $\xi$ e $\eta$ dois fibrados vetoriais em $\mathbf{V B}_{\mathbf{B}}^{\mathbf{n}}$ e $u: \xi \longrightarrow \eta$ um B-morfismo tal que, em cada fibra, u é um isomorfismo entre espaços vetoriais. Então, u é um homeomorfismo.

Demonstração: Em cada fibra, defina $v_{\pi_{\eta}^{-1}(b)}: \pi_{\eta}^{-1}(b) \longrightarrow \pi_{\xi}^{-1}(b)$, como sendo o inverso de $u$. É suficiente mostrar que $v=\left.\bigcup_{b \in B} v\right|_{\pi_{\eta}^{-1}(b)}$ é contínua. Considere duas cartas locais

$$
h: U \times \mathbb{R}^{n} \longrightarrow \pi_{\xi}^{-1}(U) \text { e } h^{\prime}: U \times \mathbb{R}^{n} \longrightarrow \pi_{\eta}^{-1}(U)
$$

de $\xi$ e $\eta$, respectivamente. (Observe que ambas têm o mesmo domínio, para isto, basta tomar interseções.)

A aplicação $\left.h^{-1} \circ u\right|_{\pi_{\xi}^{-1}(U)} \circ h: U \times \mathbb{R}^{n} \longrightarrow U \times \mathbb{R}^{n}$ é dada por $(b, x) \longmapsto(b, f(b, x))$, no qual $f: U \times \mathbb{R}^{n} \longrightarrow \mathbb{R}^{n}$ é contínua em ambas as variáveis. Para cada $b \in U$, a função $f(b): \mathbb{R}^{n} \longrightarrow \mathbb{R}^{n}$, definida como $f(b)(x)=f(b, x)$, é uma transformação linear (neste caso é um isomorfismo) e podemos pensar em $f(b)$ como uma matriz invertível em $G L(n, \mathbb{R})$ (conjunto das matrizes reais invertíveis $n \times n)$. Assim, podemos definir $h^{\prime-1} \circ u \circ h(b, x)=(b, f(b) x)$. Com isso verificamos que $h^{-1} \circ v \circ h^{\prime}(b, x)=\left(b, f(b)^{-1} x\right)$ é a inversa de $h^{-1} \circ u \circ h$ e então é contínua. $\left.\operatorname{Logo} v\right|_{\pi_{\eta}^{-1}(U)}: \pi_{\eta}^{-1}(U) \longrightarrow \pi_{\xi}^{-1}(U)$ é contínua e, portanto, $v: E(\eta) \longrightarrow B$ é contínua

\subsection{A Variedade de Grassmann}

O exemplo mais importante de fibrado vetorial é o fibrado vetorial canônico que será definido nesta seção.

Definição 1.2.1. Um $k$-referencial de $\mathbb{R}^{n}(k \leq n)$ é uma $k$-upla de vetores linearmente independentes de $\mathbb{R}^{n}$. O conjunto de todos os $k$-referenciais de $\mathbb{R}^{n}$ forma um conjunto aberto de $\underbrace{\mathbb{R}^{n} \times \cdots \times \mathbb{R}^{n}}_{k \text { vezes }}$ chamado Variedade de Stiefel e denotado por $V_{k}\left(\mathbb{R}^{n}\right)$. 
O conjunto $V_{k}^{0}\left(\mathbb{R}^{n}\right)$ denota o subconjunto de $V_{k}\left(\mathbb{R}^{n}\right)$ formado pelas $k$-uplas ortonormais, isto é, $\left(v_{1}, \ldots, v_{k}\right) \in V_{k}^{0}\left(\mathbb{R}^{n}\right)$ quando $\left\langle v_{i}, v_{j}\right\rangle=\delta_{i j}$ (delta de Kronecker), para $i, j \in\{1, \ldots, k\}$.

Definição 1.2.2. A Variedade de Grassmann $G_{k}\left(\mathbb{R}^{n}\right)$ é o conjunto de todos os subespaços vetoriais de dimensão $k$ de $\mathbb{R}^{n}$ juntamente com a topologia quociente induzida por $q: V_{k}\left(\mathbb{R}^{n}\right) \longrightarrow$ $G_{k}\left(\mathbb{R}^{n}\right)$, no qual q associa uma $k$-upla $\left(v_{1}, \ldots, v_{k}\right)$ ao subespaço gerado $\left[v_{1}, \ldots, v_{k}\right]$.

A aplicação $\left.q\right|_{V_{k}^{0}\left(\mathbb{R}^{n}\right)}: V_{k}^{0}\left(\mathbb{R}^{n}\right) \longrightarrow G_{k}\left(\mathbb{R}^{n}\right)$ induz a mesma topologia da definição anterior. Assim, como $V_{k}^{0}\left(\mathbb{R}^{n}\right)$ é compacto, segue que $G_{k}\left(\mathbb{R}^{n}\right)$ também é compacto.

Para $k=1$, tem-se $V_{1}^{0}\left(\mathbb{R}^{n}\right)=S^{n-1}$ e $G_{1}\left(\mathbb{R}^{n}\right)=\mathbb{R} P^{n-1}$ (pois trata-se do conjunto de todas as retas de $\mathbb{R}^{n}$ com a topologia quociente induzida pela aplicação $\left.z \in S^{n-1} \longmapsto[z]=[-z]\right)$.

Naturalmente, ao identificar $\mathbb{R}^{n}$ com $\mathbb{R}^{n} \times\{0\} \subset \mathbb{R}^{n+1}$, tem-se $G_{k}\left(\mathbb{R}^{n}\right) \subset G_{k}\left(\mathbb{R}^{n+1}\right)$. Define-se $G_{k}=G_{k}\left(\mathbb{R}^{\infty}\right)=\bigcup_{n \geq k} G_{k}\left(\mathbb{R}^{n}\right)$. A topologia de $G_{k}$ é a topologia fraca, isto é, um conjunto $A$ é fechado em $G_{k}$ se, e somente se, $A \cap G_{k}\left(\mathbb{R}^{n}\right)$ for fechado, para todo natural $n$.

Exemplo 1.2.3 (Fibrado Vetorial Canônico).

Seja $E \subset G_{k}\left(\mathbb{R}^{n}\right) \times \mathbb{R}^{n}$ o conjunto dos pares $(V, x)$ tais que $x \in V$. Então, $\gamma_{k}^{n}=\left(E, \pi_{1}, G_{k}\left(\mathbb{R}^{n}\right), \mathbb{R}^{k}\right)$ é um fibrado vetorial de dimensão $k$, chamado fibrado vetorial canônico (quando $n=\infty$, denotaremos $\gamma_{k}^{\infty}$ simplesmente por $\gamma_{k}$ ). De fato: uma fibra de $\gamma_{k}^{n}$ é da forma $\{V\} \times V$, que é naturalmente isomorfo a $\mathbb{R}^{k}$, pois $V$ é um espaço vetorial $k$-dimensional. É preciso checar a condição de trivialidade local.

Sejam $L \in G_{k}\left(\mathbb{R}^{n}\right)$ fixado e $\pi_{L}$ a projeção ortogonal de $\mathbb{R}^{n}$ em L. Considere o conjunto $U_{L}=\left\{L^{\prime} \in G_{k}\left(\mathbb{R}^{n}\right) ; \pi_{L}\left(L^{\prime}\right)\right.$ possui dimensão $\left.k\right\}$. O conjunto $U_{L}$ é aberto. Com efeito, a préimagem de $U_{L}$ pela projeção canônica $q$ de $V_{k}\left(\mathbb{R}^{n}\right)$ em $G_{k}\left(\mathbb{R}^{n}\right)$ é o conjunto de todas as $k$-uplas $\left(v_{1}, \ldots, v_{k}\right)$ tais que $\pi_{L}\left(v_{1}\right), \ldots, \pi_{L}\left(v_{k}\right)$ são l.i. Seja $M$ a matriz da transformação linear $\pi_{L}$ com respeito à base canônica de $\mathbb{R}^{n}$ e uma base fixada de L. Então, $q^{-1}\left(U_{L}\right)$ se torna o conjunto das $k$-uplas $\left(v_{1}, \ldots, v_{k}\right)$ tais que a matriz com colunas $M v_{1}, \ldots, M v_{k}$ possui determinante não nulo. Como o determinante é uma função contínua segue que $q^{-1}\left(U_{L}\right)$ é aberto em $V_{k}\left(\mathbb{R}^{n}\right)$. Logo, $U_{L}$ é aberto.

Defina $h: \pi_{1}^{-1}\left(U_{L}\right) \longrightarrow U_{L} \times L \approx U_{L} \times \mathbb{R}^{k}$ por $h\left(L^{\prime}, x\right)=\left(L^{\prime}, \pi_{L}(x)\right)$. Verifica-se que $h e ́$ uma bijeção contínua e linear nas fibras. Para $L^{\prime} \in U_{L}$, existe uma única transformação linear invertível $\phi_{L^{\prime}}: \mathbb{R}^{n} \longrightarrow \mathbb{R}^{n}$, definida da seguinte forma: $\left.\phi_{L^{\prime}}\right|_{L^{\prime}}=\pi_{L}$ e $\left.\phi_{L^{\prime}}\right|_{\operatorname{ker} \pi_{L}}=I_{\operatorname{ker} \pi_{L}}$ (note 
que $\operatorname{ker} \pi_{L}=L^{\perp}$, pois $\left.\mathbb{R}^{n}=L^{\prime} \oplus L^{\perp}\right)$. Podemos descrever $\phi_{L^{\prime}}$ como o produto de duas matrizes $A \cdot D^{-1}$, no qual $D$ é uma matriz que leva a base canônica de $\mathbb{R}^{n}$ em $v_{1}, \ldots, v_{k}$ (uma base fixada de $\left.L^{\prime}\right), v_{k+1}, \ldots, v_{n}\left(\right.$ uma base fixada de $\left.L^{\perp}\right)$ e A leva a base canônica de $\mathbb{R}^{n}$ em $\pi_{L}\left(v_{1}\right), \ldots, \pi_{L}\left(v_{k}\right)$ (base de $L$ ), $v_{k+1}, \ldots, v_{n}$. A função $\phi_{L^{\prime}}$ não depende da base de $L^{\prime}$ mas depende continuamente dos vetores $v_{i}$ 's. Segue que $\phi_{L^{\prime}}$ depende continuamente de $L^{\prime}$, para $L^{\prime} \in U_{L}$, pois $G_{k}\left(\mathbb{R}^{n}\right)$ possui a topologia quociente.

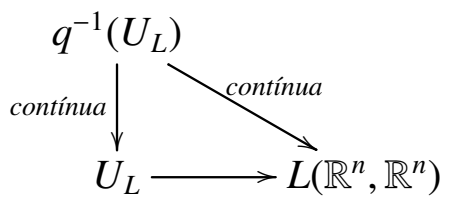

Tem-se $h\left(L^{\prime}, x\right)=\left(L^{\prime}, \pi_{L}(x)\right)=\left(L^{\prime}, \phi_{L^{\prime}}(x)\right)$ e $h^{-1}\left(L^{\prime}, w\right)=\left(L^{\prime}, \phi_{L^{\prime}}^{-1}(w)\right)$. Portanto, $h$ é uma carta local.

Para $n=\infty$, tome $L \subset \mathbb{R}^{\infty}$ um espaço vetorial de dimensão $k$ e seja $U_{L} \subset G_{k}$ o conjunto de todos os subespaços $L^{\prime}$ de dimensão $k$ tais que $\pi_{L}\left(L^{\prime}\right)=L$, no qual $\pi_{L}: \mathbb{R}^{\infty} \longrightarrow L$ é a projeção ortogonal. Tem-se que $U_{L}$ é aberto em $G_{k}$, pois $U_{L} \cap G_{k}\left(\mathbb{R}^{n}\right)$ é aberto para todo natural $n$. Também, $\left.h\right|_{\pi_{1}^{-1}\left(U_{L} \cap G_{k}\left(\mathbb{R}^{n}\right)\right)}$ definida como acima é contínua para todo natural $n$. Logo, $h=\left.\bigcup_{n} h\right|_{\pi_{1}^{-1}\left(U_{L} \cap G_{k}\left(\mathbb{R}^{n}\right)\right)}: \pi_{1}^{-1}\left(U_{L}\right) \longrightarrow U_{L} \times L \approx U_{L} \times \mathbb{R}^{k}$ é contínua. Analogamente para $h^{-1}$.

OBS 1.2.4. Uma maneira não direta (e mais simples) de mostrar que $\gamma_{k}^{n}$ é um fibrado vetorial será visto no Exemplo 1.8 .3

Definição 1.2.5. Um fibrado vetorial de dimensão 1 é chamado de fibrado vetorial em linha.

Exemplo 1.2.6 (Fibrado vetorial em linha canônico).

Considere a variedade de Grassmann $G_{1}=\mathbb{R} P^{\infty}$. O fibrado vetorial $\gamma_{1}$ recebe o nome de fibrado vetorial em linha canônico.

OBS 1.2.7. A variedade de Grassmann $G_{m}\left(\mathbb{R}^{n+m}\right)$ é uma variedade topológica compacta de dimensão nm (Vide [28. Capítulo 5, Lema 5.1, p.57]).

OBS 1.2.8. A variedade de Grassmann $G_{n}\left(\mathbb{R}^{\infty}\right)$ é um complexo $C W$. Tal estrutura celular pode ser dada de forma que os subespaços $G_{n}\left(\mathbb{R}^{n+k}\right)$ sejam subcomplexos finitos (Vide [28] Capítulo 6, Teorema 6.4 p. 79]). 


\subsection{Soma de Whitney e Fibrados Induzidos}

Nesta seção serão apresentadas formas de se obter novos fibrados vetoriais a partir de outros fibrados vetoriais dados.

Uma forma simples de se obter um fibrado vetorial a partir de outros dois fibrados vetoriais dados, é fazendo o seu produto cartesiano. De fato: se $\xi_{1}=\left(E_{1}, \pi, B_{1}, \mathbb{R}^{n}\right)$ e $\xi_{2}=\left(E_{2}, \pi^{\prime}, B_{2}, \mathbb{R}^{m}\right)$ são dois fibrados vetoriais, então $\xi \times \eta=\left(E_{1} \times E_{2}, \pi \times \pi^{\prime}, B_{1} \times B_{2}, \mathbb{R}^{n} \times \mathbb{R}^{m}\right)$ é um fibrado vetorial, no qual a condição de trivialidade local é satisfeita fazendo o produto cartesiano das cartas locais.

Outras formas mais interessantes são dadas a seguir.

Definição 1.3.1. Sejam $\xi=\left(E, \pi, B, \mathbb{R}^{n}\right)$ e $\eta=\left(E^{\prime}, \pi^{\prime}, B, \mathbb{R}^{m}\right)$ dois fibrados vetoriais com base B. A Soma de Whitney de $\xi$ e $\eta$ é o fibrado vetorial $\xi \oplus \eta=\left(E \oplus E^{\prime}, q, B, \mathbb{R}^{n} \oplus \mathbb{R}^{m} \cong \mathbb{R}^{n+m}\right)$, no qual $E \oplus E^{\prime}=\bigcup_{b \in B} \pi^{-1}(b) \times \pi^{\prime-1}(b) \subset E \times E^{\prime}$ é o conjunto dos pontos $\left(x, x^{\prime}\right)$ tais que $\pi(x)=\pi^{\prime}\left(x^{\prime}\right)$ e $q\left(x, x^{\prime}\right)=\pi(x)=\pi^{\prime}\left(x^{\prime}\right)$.

A estrutura linear de $q^{-1}(b)=\pi^{-1}(b) \times \pi^{\prime-1}(b)$ segue naturalmente de $\pi^{-1}(b) \oplus \pi^{\prime-1}(b)$. Sejam $h_{1}: U_{1} \times \mathbb{R}^{n} \longrightarrow \pi^{-1}\left(U_{1}\right), h_{2}: U_{2} \times \mathbb{R}^{n} \longrightarrow \pi^{\prime-1}\left(U_{2}\right)$ cartas locais de $\xi, \eta$, respectivamente. Tome $U=U_{1} \cap U_{2}$. A função $h=h_{1} \oplus h_{2}: U \times \mathbb{R}^{n} \oplus \mathbb{R}^{m} \longrightarrow q^{-1}(U)$, dada por, $h(b, x+y)=$ $\left(h_{1}(b, x), h_{2}(b, y)\right)$ é uma carta local para $\xi \oplus \eta$.

Seja $f: B_{1} \longrightarrow B$ uma função contínua. Dado um fibrado vetorial $\xi=\left(E, \pi, B, \mathbb{R}^{n}\right)$, pode-se associá-lo ao fibrado vetorial $f^{*}(\xi)=\left(E_{1}, \pi_{1}, B_{1}, \mathbb{R}^{n}\right)$, no qual $E_{1} \subset B_{1} \times E$ é o conjunto dos pares $\left(b_{1}, x\right)$ tais que $f\left(b_{1}\right)=\pi(x)$ e $\pi_{1}$ é projeção na primeira coordenada. Uma fibra $\left\{b_{1}\right\} \times \pi^{-1}\left(f\left(b_{1}\right)\right)$ possui naturalmente uma estrutura linear. Dada uma carta local $h: U \times \mathbb{R}^{n} \longrightarrow \pi^{-1}(U)$ de $\xi$, a função

$$
h^{*}: f^{-1}(U) \times \mathbb{R}^{n} \longrightarrow \pi_{1}^{-1}\left(f^{-1}(U)\right)
$$

dada por $h^{*}\left(b_{1}, x\right)=\left(b_{1}, h\left(f\left(b_{1}\right), x\right)\right)$ define uma carta para $f^{*}(\xi)$.

Definição 1.3.2. O fibrado vetorial $f^{*}(\xi)=\left(E_{1}, \pi_{1}, B_{1}, \mathbb{R}^{n}\right)$ é chamado fibrado vetorial induzido por $f$ de $\xi$. 
A função $f_{\xi}: E_{1} \longrightarrow E$, que leva $\left(b_{1}, x\right)$ em $x$, define um isomorfismo em nível de fibra.

Note que se $u: \xi \longrightarrow \eta$ for um $B$-morfismo, então $f^{*}(u): f^{*}(\xi) \longrightarrow f^{*}(\eta)$, definida por $f^{*}(u)\left(b_{1}, x\right)=\left(b_{1}, u(x)\right)$ é um $B_{1}$-morfismo. Assim, $f^{*}: \mathbf{V B}_{\mathbf{B}} \longrightarrow \mathbf{V B}_{\mathbf{B}_{1}}$ é um funtor covariante. Se $g: B_{2} \longrightarrow B_{1}$, tem-se $(f \circ g)^{*}(\xi)$ e $g^{*}\left(f^{*}(\xi)\right) B_{2}$-isomorfos. Quando $B_{1}=B$ e $f=I d_{B}, I d_{B}^{*}(\xi)$ e $\xi$ são $B$-isomorfos.

Exemplo 1.3.3. Sejam $\xi^{n}$ um fibrado vetorial com espaço base $B$ e $A \subset B$. Então, a inclusão $i: A \hookrightarrow B$ induz $i^{*}(\xi)=\left(E_{1}, \pi_{1}, A, \mathbb{R}^{n}\right)$. Este fibrado é isomorfo a $\xi^{\prime}=\left(E^{\prime}, \pi^{\prime}, A, \mathbb{R}^{n}\right)$, no qual $E^{\prime}=\pi_{\xi}^{-1}(A)$ e $\pi^{\prime}=\left.\pi_{\xi}\right|_{E^{\prime}}$. O fibrado vetorial $i^{*}(\xi)$ é chamado restrição de $\xi$ por $A e$ é denotado por $\left.\xi\right|_{A}$. Note que para um aberto $U \subset B$ de uma carta local de $\xi$ temos $\left.\xi\right|_{U}=$ $\left(U \times \pi_{\xi}^{-1}(U), \pi_{1}, U, \mathbb{R}^{n}\right)$.

Exemplo 1.3.4. Sejam dois fibrados vetoriais $\xi=\left(E, \pi, B, \mathbb{R}^{n}\right)$ e $\eta=\left(E^{\prime}, \pi^{\prime}, B, \mathbb{R}^{m}\right)$ e considere a função diagonal $d: B \longrightarrow B \times B$. Então, $d^{*}(\xi \times \eta)$ é B-isomorfo a $\xi \oplus \eta$.

Proposição 1.3.5. Dado um morfismo entre fibrados vetoriais $(v, f): \eta \longrightarrow \xi$, existe um único $B(\eta)$-morfismo $\omega: \eta \longrightarrow f^{*}(\xi)$ tal que $v=f_{\xi} \circ \omega$.

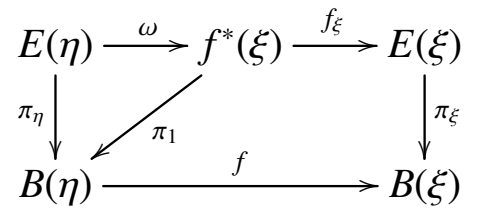

Observemos que $\omega$ é uma função da forma $\omega(y)=(a(y), b(y))$. Como deve-se ter $f_{\xi} \circ \omega(y)=$ $v(y)$, então $b(y)=v(y)$. Também, para que $\omega$ seja um morfismo devemos ter $\pi_{1} \circ \omega(y)=\pi_{\eta}(y)$. Portanto, $\omega(y)=\left(\pi_{\eta}(y), v(y)\right)$ é o único morfismo satisfazendo o enunciado da proposição.

Corolário 1.3.6. Sejam $\xi$ e $\eta$ dois fibrados vetoriais com mesma dimensão e $f: B(\eta) \longrightarrow B(\xi)$ uma função contínua. Então, $f^{*}(\xi)$ e $\eta$ são $B(\eta)$-isomorfos, se e somente se, existe um morfismo $(v, f): \eta \longrightarrow \xi$ que em cada fibra é um isomorfismo.

\subsection{Aplicação de Gauss}

Definição 1.4.1. Seja $\xi$ um fibrado vetorial de dimensão $n$. Uma função $g: E(\xi) \longrightarrow \mathbb{R}^{m}$, no qual $n \leq m \leq \infty$, é uma aplicação de Gauss se a restrição $\left.g\right|_{\pi^{-1}(b)}: \pi^{-1}(b) \longrightarrow \mathbb{R}^{m}$ for uma transformação linear injetora, para todo $b \in B$. 
O exemplo a seguir, apesar de simples, é muito importante.

Exemplo 1.4.2. A projeção na segunda coordenada $q: E\left(\gamma_{k}^{m}\right) \longrightarrow \mathbb{R}^{m}$ é uma aplicação de Gauss do k-dimensional fibrado vetorial canônico $\gamma_{k}^{m}$.

Proposição 1.4.3. Seja $(u, f): \xi^{k} \longrightarrow \gamma_{k}^{m}$ um morfismo entre fibrados vetoriais, no qual $\left.u\right|_{\pi^{-1}(b)} e ́$ um isomorfismo, para cada $b \in B$. Então, qo ué uma aplicação de Gauss de $\xi$. Reciprocamente, se $g: E(\xi) \longrightarrow \mathbb{R}^{m}$ for uma aplicação de Gauss de $\xi$, então existirá um morfismo $(u, f): \xi \longrightarrow$ $\gamma_{k}^{m}$ tal que $q \circ u=g$.

Demonstração: A primeira afirmação é imediata. Para demonstrar a segunda parte, basta definir $f: B \longrightarrow G_{k}\left(\mathbb{R}^{m}\right)$ por $f(b)=g\left(\pi^{-1}(b)\right)$ e verificar que $u(x)=(f \circ \pi(x), g(x))$ é a função procurada. Note que $u$ é um isomorfismo quando restrito a cada fibra.

O seguinte corolário é um critério de existência para aplicações de Gauss.

Corolário 1.4.4. Existe uma aplicação de Gauss $g: E\left(\xi^{k}\right) \longrightarrow \mathbb{R}^{m}$, com $k \leq m \leq \infty$ se, e somente se, existe uma função contínua $f: B(\xi) \longrightarrow G_{k}\left(\mathbb{R}^{m}\right)$ tal que $f^{*}\left(\gamma_{k}^{m}\right)$ e $\xi$ são $B(\xi)$ isomorfos.

Demonstração: Pela Proposição 1.4 .3 , existe uma aplicação de Gauss $g: E\left(\xi^{k}\right) \longrightarrow \mathbb{R}^{m}$ se, e somente se, existe um morfismo $(u, f)$ tal que $\left.u\right|_{\pi^{-1}(b)}$ é um isomorfismo, para cada $b \in B$, o que é equivalente, pelo Corolário 1.3.6 à existência de uma função contínua $f: B(\xi) \longrightarrow G_{k}\left(\mathbb{R}^{m}\right)$ tal que $f^{*}\left(\gamma_{k}^{m}\right)$ e $\xi$ são $B(\xi)$-isomorfos.

Proposição 1.4.5. [19] Capítulo 3, Proposição 5.4, p. 30]. Seja $\xi$ um fibrado vetorial com espaço base B paracompacto $]^{1}$ Então, existe uma cobertura aberta enumerável $\left\{U_{j}\right\}_{1 \leq j}$ de B tal que $\left.\xi\right|_{U_{j}}$ é trivial.

Todo fibrado vetorial com espaço base paracompacto possui uma aplicação de Gauss, como mostra o resultado a seguir.

Teorema 1.4.6. Seja $\xi=\left(E, \pi, B, \mathbb{R}^{k}\right)$ um fibrado vetorial, no qual B é paracompacto. Então, existe uma aplicação de Gauss $g: E \longrightarrow \mathbb{R}^{\infty}$.

\footnotetext{
${ }^{1}$ Vide Definição 6.6 .3
} 
Demonstração: Como $B$ é paracompacto, existe um atlas $\left\{U_{i}, h_{i}\right\}_{1 \leq i}$ indexado em um conjunto enumerável. Também, pode-se assumir que a cobertura aberta $\left\{U_{i}\right\}_{1 \leq i}$ seja localmente finita e que exista uma partição da unidade $\mathrm{2}^{2}\left\{\eta_{i}\right\}_{1 \leq i}$ subordinada à cobertura $\left\{U_{i}\right\}_{1 \leq i}$.

Considere $g_{i}: E \longrightarrow \mathbb{R}^{k}$, no qual $\left.g_{i}\right|_{\pi^{-1}\left(U_{i}\right)}=\left(\eta_{i} \circ \pi\right) \cdot\left(\pi_{2} \circ h_{i}^{-1}\right)\left(\pi_{2}\right.$ é a projeção na segunda coordenada de $U_{i} \times \mathbb{R}^{k}$ em $\left.\mathbb{R}^{k}\right)$ e $\left.g_{i}\right|_{E-\pi^{-1}\left(U_{i}\right)} \equiv 0$, para todo $i \geq 1$. Defina $g: E \longrightarrow \bigoplus_{1 \leq i} \mathbb{R}^{k}$ por $g(x)=\sum_{1 \leq i} g_{i}(x)$. É fácil verificar que $g$ é uma aplicação de Gauss.

Imediatamente, do Teorema 1.4.6 e do Corolário 1.4.4, obtém-se o resultado a seguir.

Corolário 1.4.7. Todo fibrado vetorial $\xi^{k}$ com espaço base paracompacto B é B-isomorfo a $f^{*}\left(\gamma_{k}\right)$, para alguma função contínua $f: B \longrightarrow G_{k}\left(\mathbb{R}^{\infty}\right)$.

\subsection{Métrica em um fibrado vetorial}

Nesta seção será apresentado o conceito de métrica em um fibrado vetorial. O resultado central desta seção é o fato de que todo fibrado vetorial com espaço base paracompacto possui uma métrica.

Definição 1.5.1. Seja $\xi=\left(E, \pi, B, \mathbb{R}^{n}\right)$ um fibrado vetorial. Uma métrica em $\xi$ é uma função contínua $\beta: E \oplus E \longrightarrow \mathbb{R}$ tal que $\left.\beta\right|_{\pi^{-1}(b) \oplus \pi^{-1}(b)}$ é um produto interno, para cada $b \in B$.

Denota-se $\beta(x, x)$ por $\|x\|_{\beta}$.

\section{Exemplos 1.5.2.}

(i) Seja $\theta^{n}=\left(B \times \mathbb{R}^{n}, \pi_{1}, B, \mathbb{R}^{n}\right)$ o fibrado vetorial trivial. Então, $\beta:\left(B \times \mathbb{R}^{n}\right) \oplus\left(B \times \mathbb{R}^{n}\right) \longrightarrow \mathbb{R}$, definida por $\beta((b, x),(b, y))=\langle x, y\rangle$ (produto interno canônico em $\left.\mathbb{R}^{n}\right)$, é uma métrica em $\theta^{n}$.

(ii)Para $k \leq n \leq \infty, \beta: E\left(\gamma_{k}^{n}\right) \oplus E\left(\gamma_{k}^{n}\right) \longrightarrow \mathbb{R}$ definida por $\beta((V, x),(V, y))=\langle x, y\rangle$ (produto interno canônico em $\mathbb{R}^{n}$ ), é uma métrica em $\gamma_{k}^{n}$.

Teorema 1.5.3. Todo fibrado vetorial $\xi^{k}$ com aplicação de Gauss $g: E(\xi) \longrightarrow \mathbb{R}^{\infty}$ possui uma métrica.

\footnotetext{
${ }^{2}$ Vide Definição 6.6 .6
} 
Demonstração: Defina $\beta: E(\xi) \oplus E(\xi) \longrightarrow \mathbb{R}$ por $\beta(x, y)=\langle g(x), g(y)\rangle$ (produto interno canônico em $\mathbb{R}^{\infty}$ ), para todo $(x, y) \in \pi_{\xi}^{-1}(b) \oplus \pi_{\xi}^{-1}(b)$, com $b \in B$. Como $g$ é contínua, linear e injetora nas fibras, segue que $\beta$ é uma métrica em $\xi$.

OBS 1.5.4. Segue dos Teoremas 1.4.6 e 1.5.3 que todo fibrado vetorial com espaço base paracompacto possui uma métrica.

Definição 1.5.5. Seja $\xi=\left(E, \pi, B, \mathbb{R}^{n}\right)$ um fibrado vetorial. Uma seção de $\xi$ é uma função contínua $s: B \longrightarrow$ E tal que $\pi \circ s=I d_{B}$.

Note que $s(b) \in \pi^{-1}(b)$, para todo $b \in B$.

Exemplo 1.5.6. Seja $\theta^{n}=\left(B \times \mathbb{R}^{n}, \pi_{1}, B, \mathbb{R}^{n}\right)$ o fibrado trivial. Então, definimos as seções $s_{i}: B \longrightarrow B \times \mathbb{R}^{n}$ por $s_{i}(b)=\left(b, e_{i}\right)$, para $i \in\{1, \ldots, n\}$, no qual $\left\{e_{1}, \ldots, e_{n}\right\}$ é a base canônica de $\mathbb{R}^{n}$. Note que $s_{1}(b), \ldots, s_{n}(b)$ são vetores linearmente independentes, para todo $b \in B$.

Lema 1.5.7. Sejam $\xi=\left(E, \pi, B, \mathbb{R}^{n}\right)$ um fibrado vetorial com métrica $\beta$ e $s_{1}, \ldots, s_{m}$ m seções de $\xi$ linearmente independentes, isto é, $s_{1}(b), \ldots, s_{m}(b)$ formam um conjunto linearmente independente em $\pi^{-1}(b)$, para todo $b \in B$. Então, existem $m$ seções $s_{1}^{*}, \ldots, s_{m}^{*}$ de $\xi$, que são combinações lineares de $s_{1}, \ldots, s_{m}$, tais que $\beta\left(s_{i}^{*}(b), s_{j}^{*}(b)\right)=\delta_{i j}$ (delta de Kronecker), para todo $i, j \in\{1, \ldots, m\}$ e $b \in B$.

Demonstração: Defina $s_{1}^{*}(b)=\frac{s_{1}(b)}{\left\|s_{1}(b)\right\|_{\beta}}$ e $s_{k}^{*}(b)=\frac{s_{k}(b)-\sum_{1 \leq j \leq k-1} \beta\left(s_{k}(b), s_{j}^{*}(b)\right) s_{j}^{*}(b)}{\left\|s_{k}(b)-\sum_{1 \leq j \leq k-1} \beta\left(s_{k}(b), s_{j}^{*}(b)\right) s_{j}^{*}(b)\right\|_{\beta}}$, para todo $k \in\{2, \ldots, m\}$ e $b \in B$.

Proposição 1.5.8. [17 Capítulo 1, Proposição 1.3, p. 12]. Sejam $\xi=\left(E, \pi, B, \mathbb{R}^{n}\right)$ um fibrado vetorial, no qual B é paracompacto e $\eta=\left(E_{1}, \pi^{\prime}, B, \mathbb{R}^{k}\right)$ um subfibrado vetorial de $\xi$. Então, existe um subfibrado vetorial $\eta^{\perp}$ de $\xi$ tal que $\xi$ é isomorfo a $\eta \oplus \eta^{\perp}$. O fibrado vetorial $\eta^{\perp} e ́$ chamado de complemento ortogonal de $\eta \mathrm{em} \xi$

Exemplo 1.5.9. Sejam $\theta=\left(S^{n} \times \mathbb{R}^{n+1}, \pi_{1}, S^{n}, \mathbb{R}^{n+1}\right)$, $\tau\left(S^{n}\right)$ e $\eta\left(S^{n}\right)$ como no Exemplo 1.1 .4 Então, $\theta \cong \tau\left(S^{n}\right) \oplus \eta\left(S^{n}\right)$. 


\subsection{Fibrados associados}

O fibrado em esfera associado a um fibrado vetorial será o principal objeto de estudo para a generalização do teorema de Borsuk-Ulam. O fibrado projetivo associado será importante na construção das classes de Stiefel-Whitney.

Teorema 1.6.1. Seja $\xi=\left(E, \pi, B, \mathbb{R}^{n}\right)$ um fibrado vetorial com métrica $\beta$. Então, $\xi$ possui um atlas $\left\{\left(V_{i}, h_{i}^{*}\right)\right\}_{i}$ tal que $\langle x, y\rangle=\beta\left(h_{i}^{*}(b, x), h_{i}^{*}(b, y)\right)$, para cada $x, y \in \mathbb{R}^{n}$ e $b \in V_{i}$.

Demonstração: Seja $\left\{V_{i}, h_{i}\right\}$ um atlas de $\xi$. Para cada $i$, existem $n$ seções linearmente independentes $s_{j}: V_{i} \longrightarrow \pi^{-1}\left(V_{i}\right)$ de $\left.\xi\right|_{V_{i}}$ definidas por $s_{j}(b)=h_{i}\left(b, e_{j}\right)$, para $j \in\{1, \ldots, n\}$, no qual $e_{1}, \ldots, e_{n}$ formam a base canônica de $\mathbb{R}^{n}$. Logo, pelo Lema 1.5.7, existem $n$ seções de $\left.\xi\right|_{V_{i}}$, $s_{1}^{*}, \ldots, s_{n}^{*}$, tais que $\beta\left(s_{i}^{*}(b), s_{j}^{*}(b)\right)=\delta_{i j}$, para cada $b \in V_{i}$. Defina $h_{i}^{*}: V_{i} \times \mathbb{R}^{n} \longrightarrow \pi^{-1}\left(V_{i}\right)$ por $h_{i}^{*}\left(b,\left(a_{1}, \ldots, a_{n}\right)\right)=a_{1} s_{1}^{*}(b)+\cdots+a_{n} s_{n}^{*}(b)$. Então, $h_{i}^{*}$ define uma carta local para $\xi$ e mostra-se que $\beta\left(h_{i}^{*}(b, x), h_{i}^{*}(b, y)\right)=\langle x, y\rangle$.

Definição 1.6.2. Seja $\xi=\left(E, \pi, B, \mathbb{R}^{n}\right)$ um fibrado vetorial com métrica $\beta$ e atlas $\left\{V_{i}, h_{i}\right\}_{i}$. O conjunto de todos os pontos $x$ de E tais que $\|x\|_{\beta}=1$ é denotado por $S(E)$. O fibrado em esfera associado de $\xi$ é uma quádrupla $S(\xi)=\left(S(E), \pi^{\prime}, B, S^{n-1}\right)$ juntamente com o atlas $\left\{V_{i},\left.h_{i}^{*}\right|_{V_{i} \times S^{n-1}}\right\}_{i}$ (obtido como no Teorema 1.6.1), no qual $\pi^{\prime}$ é a restrição de $\pi$ por $S(E)$.

Sejam $\xi=\left(E, \pi, B, \mathbb{R}^{n}\right)$ um fibrado vetorial e $E_{0}=\bigcup_{b \in B}^{\cdot}\left(\pi^{-1}(b)-\left\{0_{b}\right\}\right)$, no qual $0_{b}$ é o vetor nulo em $\pi^{-1}(b)$. Em $E_{0}$, considere a seguinte relação: $x \sim y$, quando $\pi(x)=\pi(y)$ e $x=\lambda y$, para algum $\lambda \in \mathbb{R}-\{0\}$. O conjunto $\frac{E_{0}}{\sim}$ das classes $[x]$ é denotado por $P(E)$. A quádrupla $P(\xi)=\left(P(E), \pi^{\prime}, B, \mathbb{R} P^{n-1}\right)$, com $\pi^{\prime}([x])=\pi(x)$, satisfaz uma condição de trivialidade local, no qual $\pi^{\prime-1}(b)$ é homeomorfo a $\mathbb{R} P^{n-1}$, para todo $b \in B$. De fato, seja $h: U \times \mathbb{R}^{n} \longrightarrow \pi^{-1}(U)$ um homeomorfismo. Então, $h^{\prime}: U \times \mathbb{R} P^{n-1} \longrightarrow \pi^{-1}(U)$, definida por $h^{\prime}(b,[x])=[h(b, x)]$ é um homeomorfismo e faz o seguinte diagrama comutar.

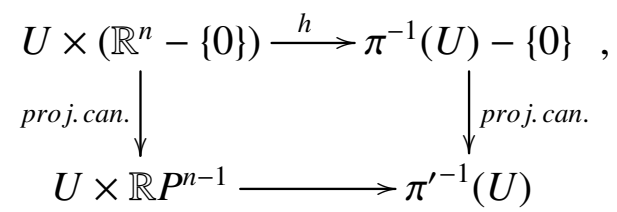

no qual proj. can. significa projeção canônica. 
Definição 1.6.3. A quádrupla $P(\xi)=\left(P(E), \pi^{\prime}, B, \mathbb{R} P^{n-1}\right)$ é chamada fibrado projetivo associado de $\xi$.

\subsection{Fibrado Vetorial Universal}

Esta seção mostrará a importância do fibrado vetorial canônico. A seguir, apresentamos dois importantes resultados, cujas demonstrações podem ser encontradas em Husemoller [19].

Teorema 1.7.1. [19. Capítulo 3, Teorema 4.7, p. 39]. Sejam $f, g: B \longrightarrow B^{\prime}$ duas funções homotópicas, no qual B é um espaço paracompacto. Se $\xi$ é um fibrado vetorial sobre B', então $f^{*}(\xi)$ e $g^{*}(\xi)$ são B-isomorfos.

Corolário 1.7.2. Seja $\xi^{n}$ um fibrado vetorial com espaço base B paracompacto e contrátil. Então, $\xi$ é trivial.

Demonstração: Seja $g: B \longrightarrow B$ uma aplicação constante. Tem-se $g^{*}(\xi) B$-isomorfo ao fibrado vetorial trivial $\left(B \times \mathbb{R}^{n}, \pi, B, \mathbb{R}^{n}\right)$. Como $I d_{B}$ e $g$ são homotópicas, segue do teorema anterior que $g^{*}(\xi)$ e $I d_{B}^{*}(\xi)$ (que é $B$-isomorfo a $\xi$ ) são $B$-isomorfos.

Teorema 1.7.3. [19. Capítulo 3, Teorema 6.2, p. 32]. Sejam B paracompacto e $f, g: B \longrightarrow$ $G_{k}\left(\mathbb{R}^{\infty}\right)$. Então, $f^{*}\left(\gamma_{k}\right)$ e $g^{*}\left(\gamma_{k}\right)$ são B-isomorfos se, e somente se, $f$ e g são homotópicos.

Definição 1.7.4. Seja $\mathbf{P}$ a categoria cujos objetos são os espaços paracompactos e os morfismos são as classes de homotopia de funções entre estes espaços. Dado $B \in \mathbf{P}$, $\operatorname{Vet}_{\mathbf{n}}(\mathbf{B})$ denotará o conjunto de todas as classes de B-isomorfismo entre fibrados vetoriais n-dimensionais com espaço base B. Um elemento de $\mathbf{V e t}_{\mathbf{n}}(\mathbf{B})$ será denotado por $\{\xi\}=\left\{\eta \in \mathbf{V B}_{\mathbf{B}}^{\mathbf{n}}\right.$; $\xi$ e $\eta$ são B-isomorfos $\}$. Se $[f]: B_{1} \longrightarrow$ B for um morfismo em $\mathbf{P}$, define-se

$$
\begin{aligned}
\operatorname{Vet}_{n}([f]): \operatorname{Vet}_{\mathbf{n}}(\mathbf{B}) & \longrightarrow \operatorname{Vet}_{\mathbf{n}}\left(\mathbf{B}_{\mathbf{1}}\right) \\
\{\xi\} & \longmapsto\left\{f^{*}(\xi)\right\} .
\end{aligned}
$$

Note que se $\{\xi\}=\{\eta\}$, então $f^{*}(\xi)$ e $f^{*}(\eta)$ são $B$-isomorfos. Também, se $[f]=[g]$, então $f^{*}(\xi)$ e $g^{*}(\xi)$ são $B$-isomorfos. Logo, a função $V e t_{n}$ está bem definida.

Proposição 1.7.5. [19. Capítulo 3, Proposição 7.1, p. 33]. Vet $t_{n}: \mathbf{P} \longrightarrow$ SET é um funtor contravariante, no qual SET é a categoria dos conjuntos e suas funções. 
Definição 1.7.6. Para cada $B \in \mathbf{P}$, define-se $\phi_{B}:\left[B, G_{k}\right] \longrightarrow \operatorname{Vet}_{n}(B)$ por $[f] \mapsto\left\{f^{*}\left(\gamma_{k}\right)\right\}$, no qual $\left[B, G_{k}\right]$ é o conjunto de todas as classes de homotopias de funções $B \longrightarrow G_{k}$.

OBS 1.7.7. Pelo Teorema 1.4.6 e Corolário 1.4.7 $\phi_{B}$ é sobrejetora e, pelo Teorema 1.7.3 $\phi_{B} e ́$ injetora.

Pelo fato de conseguirmos obter todos os fibrados vetoriais $k$-dimensionais através de $\gamma_{k}$, este recebe uma nova nomenclatura.

Definição 1.7.8. O fibrado vetorial $\gamma_{k}$ é chamado fibrado vetorial universal .

\subsection{Kernel, Imagem e Cokernel}

Sejam $\xi$ e $\eta$ dois fibrados vetoriais com espaço base $B$ e $u: \xi \longrightarrow \eta$ um $B$-morfismo.

Considere os espaços:

- $E_{1}=\left\{x \in E(\xi) ; u(x)=0\right.$ em $\left.\pi_{\eta}(x)\right\}$, no qual 0 representa o vetor nulo em $\pi^{-1}(\pi(x))$;

- $E_{2}=\{y \in E(\eta) ; y=u(x)$, para algum $x \in E(\xi)\}$

- $E_{3}$ é o conjunto quociente da seguinte relação (de equivalência) em $E(\eta): y \sim y^{\prime}$ se, e somente se, $\pi_{\eta}(y)=\pi_{\eta}\left(y^{\prime}\right)$ e $y-y^{\prime}=u(x)$, para algum $x \in E(\xi)$.

Observe que $E_{1}=\bigcup_{b \in B} \operatorname{ker}\left(u_{b}\right)$, no qual $u_{b}=\left.u\right|_{\pi_{\xi^{-1}}(b)}: \pi_{\xi}^{-1}(b) \longrightarrow \pi_{\eta}^{-1}(b)$, para todo $b \in B$. O conjunto $E_{2}$ é $\bigcup_{b \in B}^{\cdot} \operatorname{Img}\left(u_{b}\right)$ e $E_{3}=\bigcup_{b \in B}^{\cdot} \frac{\pi_{\eta}^{-1}(b)}{\operatorname{Img}\left(u_{b}\right)}=\bigcup_{b \in B}^{\cdot} \operatorname{Coker}\left(u_{b}\right)$.

Definição 1.8.1. Dizemos que u tem posto constante $k$ se $u_{b}$ possui posto $k$, para todo $b \in B$.

Teorema 1.8.2. Seja $u: \xi^{n} \longrightarrow \eta^{m}$ um B-morfismo entre fibrados vetoriais com posto constante k. As funções $p_{1}=\left.\pi_{\xi}\right|_{E_{1}}: E_{1} \longrightarrow B, p_{2}=\left.\pi_{\eta}\right|_{E_{2}}: E_{2} \longrightarrow B$ e $p_{3}: E_{3} \longrightarrow B$, definida por $p_{3}[y]=\pi_{\eta}(y)$, induzem fibrados vetoriais sobre B, os quais serão denotados por $\operatorname{ker}(u), \operatorname{Img}(u)$ e Coker(u), respectivamente.

Demonstração: Podemos supor $u: B \times \mathbb{R}^{n} \longrightarrow B \times \mathbb{R}^{m}$ dado por $u(b, x)=\left(b, u_{b}(x)\right)$, no qual $u_{b}: \mathbb{R}^{n} \longrightarrow \mathbb{R}^{m}$ é uma transformação linear. Fixe $a \in B$ e considere as notações: $\operatorname{ker}\left(u_{a}\right)=V_{2}$, 
$V_{1}=V_{2}^{\perp}, \operatorname{Img}\left(u_{a}\right)=W_{1}, W_{2}=W_{1}^{\perp}$. Então, $\mathbb{R}^{n}=V_{1} \oplus V_{2}$ e $\mathbb{R}^{m}=W_{1} \oplus W_{2}$. Também, $\operatorname{dim} V_{1}=\operatorname{dim} W_{1}=k, \operatorname{dim} V_{2}=n-k$ e $\operatorname{dim} W_{2}=m-k$ (teorema do Núcleo e Imagem).

Para cada $b \in B$, defina $\gamma_{b}: W=\mathbb{R}^{n} \oplus W_{2} \longrightarrow V=\mathbb{R}^{m} \oplus V_{2}$ da seguinte forma: $\left.\gamma_{b}\right|_{V_{1}}=\left.u_{b}\right|_{V_{1}} \oplus$ $0,\left.\gamma_{b}\right|_{V_{2}}=\left.u_{b}\right|_{V_{2}} \oplus I d_{V_{2}}$ e $\left.\gamma_{b}\right|_{W_{2}}=0 \oplus I d_{W_{2}} \oplus 0$. De outra forma, considerando $q: V_{1} \oplus V_{2} \longrightarrow V_{2}$, $q(x, y)=y$, então $\gamma_{b}((x, y), z)=\left(u_{b}(x, y)+z, q(x, y)\right)$.

Note que $\gamma_{a}$ é um isomorfismo. De fato: $\gamma_{a}: W \longrightarrow V$ é injetora por construção, como $\operatorname{dim} W=n+m-k=\operatorname{dim} V$, segue que $\gamma_{a}$ é isomorfismo.

É possível mostrar que $B \longrightarrow L(W, V)$ (conjunto das transformações lineares de $W$ em $V$ ), tal que $b \mapsto \gamma_{b}$, é contínua. Como $G L(W, V) \subset L(W, V)$ (conjunto dos isomorfismo de $W$ em $L$ ) é aberto, existe uma vizinhança aberta $U \subset B$ de $a$ tal que $\gamma_{b}$ é isomorfismo, para todo $b \in U$. Denote por $v_{b}$ a inversa de $\gamma_{b}$, para todo $b \in B$.

- $\operatorname{ker}(u)$ é um fibrado vetorial de dimensão $n-k$.

Para isto basta mostrar a condição de trivialidade. Para $a \in B$, tem-se:

Note que $u_{b}\left(x_{1}, x_{2}\right)=0$, então $\gamma_{b}\left(\left(x_{1}, x_{2}\right), 0\right)=\left(0+0, q\left(x_{1}, x_{2}\right)\right)=x_{2}$ e $\left(\left(x_{1}, x_{2}\right), 0\right)=$ $v_{b}\left(x_{2}\right)$. Agora se $\gamma_{b}\left(\left(x_{1}, x_{2}\right), 0\right)=\left(0,0, x_{2}\right)$, então $u_{b}\left(x_{1}, x_{2}\right)=0$. Portanto, $v_{b}\left(V_{2}\right)=$ $\operatorname{ker}\left(u_{b}\right) \oplus 0$.

Defina $h: U \times\{0\} \oplus V_{2} \longrightarrow p_{1}^{-1}(U)$ por $h(b,(0, x))=\left(b, v_{b}((0, x), 0)\right.$. A inversa de $h$ é $h^{-1}\left(b,\left(x_{1}, x_{2}\right)\right)=\left(b, \gamma_{b}\left(\left(x_{1}, x_{2}\right), 0\right)\right)$. Então, $h$ é um homeomorfismo que é isomorfismo nas fibras.

- $\operatorname{Img}(u)$ é um fibrado vetorial de dimensão $k$.

Note que $u_{b}\left(x_{1}, 0\right)=0$ se, e somente se, $\gamma_{b}\left(x_{1}, 0,0\right)=0$. Como $\left.\gamma_{b}\right|_{V_{1}}$ é injetora, segue que $\left.u_{b}\right|_{V_{1}}: V_{1} \longrightarrow \operatorname{Img}\left(u_{b}\right)$ é isomorfismo. Defina $h: U \times V_{1} \oplus 0 \longrightarrow p_{2}^{-1}(U)$ por $h\left(b, x_{1}, 0,0\right)=\left(b, u_{b}\left(x_{1}, 0\right), 0\right)$. A inversa de $h$ é $h^{-1}(b,(y, 0,0))=\left(b, v_{b}((y, 0,0))\right)$. Então, $h$ é uma carta para $\operatorname{Img}(u)$.

- $\operatorname{Coker}(u)$ é um fibrado vetorial de dimensão $m-k$.

Note que $\operatorname{Img}\left(u_{b}\right) \cap W_{2}=\{0\}$. A aplicação quociente $h: U \times\{0\} \times\{0\} \times W_{2} \longrightarrow p_{3}^{-1}(U)$, definida por $h\left(b, w_{2}\right)=\left(b,\left[w_{2}\right]\right)$ é um monomorfismo (logo isomorfismo) em nível de 
fibras $\left(\operatorname{se}\left(b,\left[w_{1}\right]\right)=\left(b,\left[w_{2}\right]\right)\right.$, então $\left.w_{1}-w_{2} \in \operatorname{Img}\left(u_{b}\right), \operatorname{logo} w_{1}=w_{2}\right)$. Então, $h$ é uma carta.

Exemplo 1.8.3. Seja L um subespaço $k$-dimensional de $\mathbb{R}^{n}$ gerado por $\left(v_{1}, \ldots, v_{k}\right) \in V_{k}\left(\mathbb{R}^{n}\right)$. Considere $\pi_{L}: \mathbb{R}^{n} \longrightarrow \mathbb{R}^{n}$, dada por $\pi_{L}(x)=\sum_{j=1}^{k}\left\langle x, v_{j}\right\rangle v_{j}$ (projeção ortogonal em $L$ ). A função $G_{k}\left(\mathbb{R}^{n}\right) \longrightarrow L\left(\mathbb{R}^{n}, \mathbb{R}^{n}\right), L \mapsto \pi_{L}$ é contínua, e u $: G_{k}\left(\mathbb{R}^{n}\right) \times \mathbb{R}^{n} \longrightarrow G_{k}\left(\mathbb{R}^{n}\right) \times \mathbb{R}^{n}$, definida por $(L, x) \mapsto\left(L, \pi_{L}(x)\right)$ é um $G_{k}\left(\mathbb{R}^{n}\right)$-morfismo de posto constante $k$. A imagem de u é $E\left(\gamma_{n}^{k}\right)$.

Corolário 1.8.4. Se $u: \xi^{n} \longrightarrow \eta^{m}$ for injetora em cada fibra, então Img(u) e Coker(u) serão fibrados vetoriais. Agora, se u for sobrejetora em cada fibra, então ker(u) será um fibrado vetorial.

Definição 1.8.5. Uma sequência de B-morfismos entre fibrados vetoriais

$$
\cdots \longrightarrow \stackrel{u}{\longrightarrow} \eta \stackrel{v}{\longrightarrow} \zeta \longrightarrow \cdots
$$

será uma sequência exata quando $\operatorname{Img}(u)=\operatorname{ker}(v)$.

OBS 1.8.6. $S e\left(B, I d_{b}, B\right)=0 \longrightarrow \xi \stackrel{u}{\longrightarrow} \eta \stackrel{v}{\longrightarrow} \zeta \longrightarrow 0$ for uma sequência exata, então $\xi e$ $\operatorname{ker}(v)$ serão isomorfos e $\zeta$ e Coker(v) serão isomorfos. Tal sequência será chamada sequência exata curta.

Teorema 1.8.7. [19] Capítulo 3, Teorema 9.6, p.37]. Seja $0 \longrightarrow \xi \stackrel{u}{\longrightarrow} \eta \stackrel{v}{\longrightarrow} \zeta \longrightarrow 0$ uma sequência exata curta de fibrados vetoriais com espaço base B. Seja $\beta$ uma métrica em $\eta$. Então, existe um B-isomorfismo $\xi \oplus \zeta \longrightarrow \eta$. 


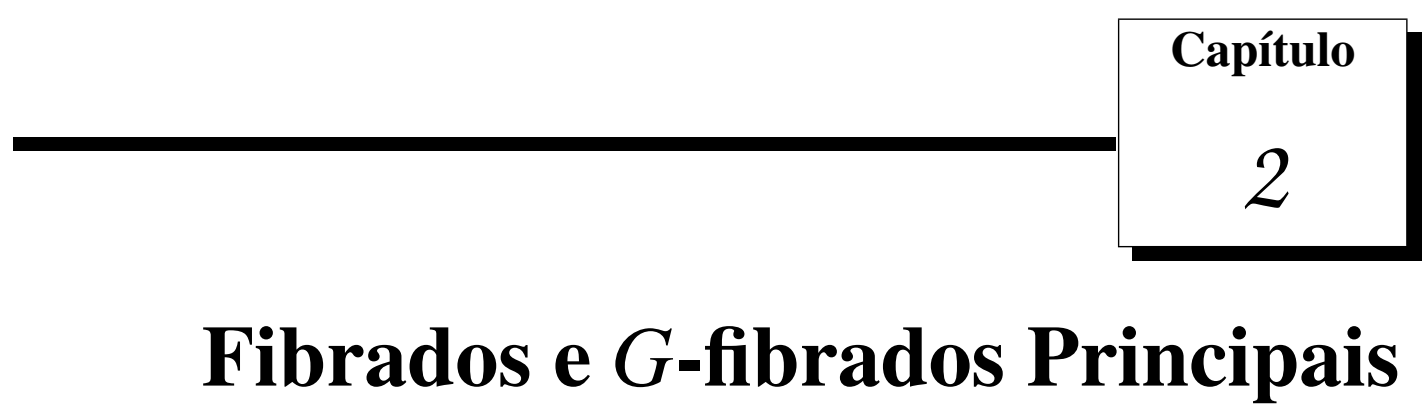

Esse capítulo destina-se à definição dos conceitos de $G$-fibrados principais e fibrados. A definição de fibrado será dada como em Husemoller [19], que diferentemente de Steenrod [35], define primeiramente $G$-fibrados principais e, posteriormente, fibrados associados. A Seção 2.1 apresenta resultados elementares da teoria de grupos topológicos, essencial para o capítulo. $\mathrm{Na}$ Seção 2.2 é definido o conceito de $G$-fibrado principal, no qual $G$ é um grupo topológico. A Seção 2.3 trata da definição de fibrado associado a um $G$-fibrado principal. Na Seção 2.4 são apresentados o conceito de fibrado coordenado e uma maneira de se obter fibrados através de funções conhecidas como funções transições. Também é vista a relação entre fibrados e fibrados vetoriais.

\subsection{Grupos Topológicos}

Nesta seção serão apresentados os conceitos elementares sobre grupos topológicos. Um grupo topológico, como veremos, nada mais é do que um grupo com uma topologia que se relaciona com sua operação.

Definição 2.1.1. Um espaço topológico de Hausdorff $G$ é um grupo topológico se possui uma estrutura de grupo multiplicativa "." tal que a função $(G \times G) \longrightarrow G$, definida por $(a, b) \mapsto a \cdot b^{-1}$, é contínua, no qual $b^{-1}$ é o inverso multiplicativo de $b$. 
O elemento neutro do grupo $G$ será denotado por $e$. Note que $(G \times G) \longrightarrow G,(a, b) \mapsto a \cdot b^{-1}$, é contínua se, e somente se, as funções $(G \times G) \stackrel{\cdot}{\longrightarrow} G,(a, b) \mapsto a \cdot b$ e, $G \stackrel{i n v}{\longrightarrow} G, a \mapsto a^{-1}$, são contínuas.

\section{Exemplos 2.1.2.}

(i) O conjunto dos números reais $\mathbb{R}$, com a operação de soma e com a topologia usual, é um grupo topológico. Também, $\mathbb{R}-\{0\}$, com a operação de multiplicação e a topologia usual, é outro exemplo de grupo topológico.

(ii) O conjunto das matrizes reais $n \times n$ invertíveis, juntamente com a operação de multiplicação de matrizes, formam o grupo linear geral $G L(n, \mathbb{R})$. Pode-se determinar uma topologia para $G L(n, \mathbb{R})$ induzida da topologia usual de $\mathbb{R}^{n^{2}}$. Assim $G L(n, \mathbb{R})$ torna-se um grupo topológico. O subgrupo $O(n)=\left\{A \in G L(n, \mathbb{R}) ; A \cdot A^{t}=I e \operatorname{det}(A)= \pm 1\right\}$ de $G L(n, \mathbb{R})$, chamado de grupo ortogonal, também é um grupo topológico.

(iii) O grupo $\mathbb{Z}_{2}$, com a topologia discreta, é um grupo topológico.

Definição 2.1.3. Sejam G um grupo topológico e $X$ um espaço topológico. Uma ação à direita de $G$ em $X$ é uma função contínua $\alpha: X \times G \longrightarrow X$ tal que:

(1) $\alpha(\alpha(x, g), h))=\alpha(x, g \cdot h)$, para todo $x \in X$ e $g, h \in G$;

(2) $\alpha(x, e)=x$, para todo $x \in X$.

O espaço X é chamado de G-espaço à dineita. Diz-se também que G age em X.

Por simplicidade, denota-se $\alpha(x, g)$ por $x g$. Assim, reescreve-se (1) e (2) como $(x g) h=x(g h)$ e $x e=x$. Pode-se definir, de maneira análoga, uma ação à esquerda de $G \operatorname{em~} X$. A terminologia $G$-espaço se referirá aos $G$-espaços à direita, a menos que se diga o contrário.

Definição 2.1.4. Sejam $G$ um grupo topológico e $X$ e $Y$ dois $G$-espaços à direita. Uma função contínua $f: X \longrightarrow Y$ tal que $f(x g)=f(x) g$, para todo $g \in G, x \in X$ e $y \in Y$, é chamada aplicação $G$-equivariante.

Os $G$-espaços e as aplicações $G$-equivariantes formam uma categoria. 
Definição 2.1.5. Seja $X$ um $G$-espaço. Dados dois elementos $x, y \in X$, diz-se que xé equivalente a $y$, quando existir $g \in G$ tal que $x=y g$.

A definição acima define uma relação de equivalência em $X$. A classe de equivalência de um elemento $x \in X$ é denotada por $x G=\{x g ; g \in G\}$ e é chamada de órbita de $x$. O conjunto de todas as órbitas de $X$ será denotado por $X \bmod G$. Pode-se induzir a topologia quociente em $X \bmod G$, isto é, a topologia induzida pela projeção canônica $X \longrightarrow X \bmod G$.

Para um $G$-espaço $X$, seja $\theta_{g}: X \longrightarrow X$ a função definida por $\theta_{g}(x)=x g$

Proposição 2.1.6. A função $\theta_{g}$ é um homeomorfismo e a projeção canônica $\varphi_{\text {can }}: X \longrightarrow X$ mod G é uma aplicação aberta.

Demonstração: A função $\theta_{g}$ é a restrição da ação $\alpha$ ao fechado $X \times\{g\}$, por isso é contínua. É fácil ver que $\theta_{g^{-1}}$ é a função inversa de $\theta_{g}$. Logo $\theta_{g}$ é um homeomorfismo. Para verificar que $\varphi_{c a n}$ é um aplicação aberta, basta observar que $\varphi_{c a n}^{-1}\left(\varphi_{c a n}(U)\right)=\bigcup_{g \in G} \theta_{g}(U)$, para cada subconjunto aberto $U \subset X$. Como $\theta_{g}(U)$ é aberto, segue que $\varphi_{c a n}^{-1}\left(\varphi_{c a n}(U)\right)$ é aberto. Logo, $\varphi_{c a n}(U)$ é aberto.

Definição 2.1.7. Sejam $G$ um grupo topológico, $X$ um $G$-espaço e $\alpha: X \times G \longrightarrow X$ uma ação de G em X. Então:

1. $\alpha$ é trivial se $x g=x$, para todo $g \in G$ e $x \in X$;

2. $\alpha$ é efetiva se $x g=x$, para todo $x \in X$, somente para $g=e \in G$;

3. $\alpha$ é livre se $x g \neq x$, para todo $g \in G-\{$ e $\}$ e $x \in X$;

Diz-se que G age trivialmente (respectivamente, efetivamente ou livremente) em $X$ e que Xé um G-espaço trivial (respectivamente, efetivo ou livre) se 1 (respectivamente, 2 ou 3) vale.

\subsection{A categoria PB}

Definição 2.2.1. Seja $X$ um $G$-espaço livre. Considere $X^{*}=\{(x, x g) \in X \times X ; g \in G\}$. A função $\tau: X^{*} \longrightarrow G$, definida por $\tau(x, x g)=g$ é chamada função translação. 
A função translação possui algumas propriedades:

1. $\tau(x, x)=e$, para todo $x \in X$;

2. $\tau(x, x g) \cdot \tau(x g,(x g) h)=\tau(x, x(g h))$, para todo $g, h \in G$ e $x \in X$;

3. $\tau(x g, x)=\tau(x, x g)^{-1}$, para todo $x \in X$ e $g \in G$.

Definição 2.2.2. Seja $X$ um G-espaço livre e suponha que a função translação $\tau: X^{*} \longrightarrow G$ seja contínua. Um $G$-fibrado principal sobre B é uma função contínua $p: X \longrightarrow B$ tal que o diagrama abaixo é comutativo.

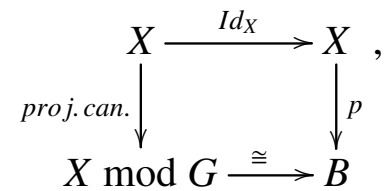

no qual $\cong$ indica que existe um homeomorfismo entre o espaço $B$ e $X \bmod G$ Denota-se o $G$ fibrado acima por $\xi=(X, p, B, G)$.

OBS 2.2.3. As mesmas terminologias usadas para fibrados vetoriais são usadas para G-fibrados principais. Neste caso, X é o espaço total, B é o espaço base, p é a aplicação projeção e $p^{-1}(b)$ é a fibra sobre b de $\xi$. Também, $X(\eta), B(\eta)$ e $p_{\eta}$ representam as componentes de um G-fibrado principal $\eta$.

Exemplo 2.2.4 ( $G$-fibrado principal trivial).

Sejam B um espaço topológico e $G$ um grupo topológico. Então, $(B \times G) \times G \longrightarrow(B \times G)$, definida por $((b, g), h) \mapsto(b, g h)$ é uma ação de $G$ em $(B \times G)$. Note que esta ação é livre. A função translação $\tau:(B \times G)^{*} \longrightarrow G, \tau((b, g),(b, h))=g^{-1} \cdot h$ é contínua e $(B \times G) \bmod G \longrightarrow B$, $(b, g) G \mapsto b$, é um homeomorfismo. Então, $\pi_{1}:(B \times G) \longrightarrow B$ é um G-fibrado principal, chamado G-fibrado principal trivial.

Exemplo 2.2.5. Seja $G=\mathbb{Z}_{2}$. A esfera unitária n-dimensional $S^{n}$ é um $\mathbb{Z}_{2}$-espaço, no qual a ação é dada pela aplicação antipodal $S^{n} \times \mathbb{Z}_{2} \longrightarrow S^{n},(x, \pm 1) \mapsto( \pm) x$. Então, $S^{n} \longrightarrow$ $S^{n} \bmod \mathbb{Z}_{2}=\mathbb{R} P^{n}$ é um $\mathbb{Z}_{2}$-fibrado principal.

Proposição 2.2.6. Seja $\xi=(X, p, B, G)$ um $G$-fibrado principal. Então, para todo $b \in B, p^{-1}(b)$ é homeomorfo a $G$. 
Demonstração: Fixe $x_{0} \in X$ e defina $u: G \longrightarrow p^{-1}(b)$, por $u(g)=x_{0} g$. Note que $x_{0} g \in p^{-1}(b)$, pois $p\left(x_{0} g\right)=p\left(x_{0}\right)$. Observe que $p^{-1}(b)$ é a órbita de $x_{0}$. A função $v: p^{-1}(b) \longrightarrow G$, definida por $v(x)=\tau\left(x_{0}, x\right)$, no qual $\tau$ é a função translação, é a inversa de $u$. Como $u$ e $v$ são contínuas, segue que $p^{-1}(b)$ e $G$ são homeomorfos.

Definição 2.2.7. Sejam $\xi=(X, p, B, G)$ e $\eta=\left(Y, q, B^{\prime}, G\right)$ dois G-fibrados principais. Um morfismo entre $G$-fibrados principais é um par de funções contínuas $(u, f): \xi \longrightarrow \eta$, no qual $u: X \longrightarrow Y$ e $f: B \longrightarrow B^{\prime}$, tal que o diagrama $X \stackrel{u}{\longrightarrow} Y$ é comutativo e u é G-equivariante.

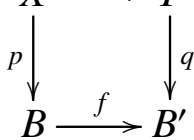

Quando $B^{\prime}=B$ e $f=I d_{B}$, $\left(u, I d_{B}\right)$ será denotado apenas por u e será chamado um $B$-morfismo.

OBS 2.2.8. A composição de morfismos entre G-fibrados principais é um morfismo entre $G$ fibrados principais. Sejam $(u, f): \xi \longrightarrow \eta e(v, g): \eta \longrightarrow \xi$ dois morfismos tais que $u \circ v=I d_{Y}$, $v \circ u=I d_{X}$. Então, dizemos que $\xi$ e $\eta$ são isomorfos. Caso $\xi$ e $\eta$ sejam G-fibrados principais sobre $B$, então dizemos que são B-isomorfos.

Definição 2.2.9. Seja $G$ um grupo topológico. A categoria cujos objetos são os G-fibrados principais e os morfismos são os morfismos entre os G-fibrados principais será denotada por PB. A subcategoria dos G-fibrados principais com espaço base B e B-morfismos será denotado por $\mathbf{P B}_{\mathbf{B}}$.

Teorema 2.2.10. [19] Capítulo 4, Teorema 3.2, p. 42] Todo morfismo em $\mathbf{P B}_{\mathbf{B}}$ é um isomorfismo.

Teorema 2.2.11. Seja $\xi=\left(B \times G, \pi_{1}, B, G\right) o G$-fibrado principal trivial. Então, os B-isomorfismos $\xi \longrightarrow \xi$ estão em correspondência biunívoca com as funções contínuas $B \longrightarrow G$.

Demonstração: Seja $g: B \longrightarrow G$ uma função contínua. Defina $h_{g}: B \times G \longrightarrow B \times G$ por $h_{g}(b, s)=(b, g(b) s)$. Note que $h_{g}((b, s) t)=h_{g}(b, s t)=(b, g(b) s t)=(b, g(b) s) t=h_{g}(b, s) t$, para $t \in G$. A função $(b, s) \mapsto\left(b, g(b)^{-1} s\right)$ define a inversa de $h_{g}$. Então, $h_{g}$ é um $B$-isomorfismo.

Agora seja $h: B \times G \longrightarrow B \times G$ um $B$-isomorfismo. Então, $h(b, s)=(b, f(b, s))$, no qual $f: B \times G \longrightarrow G$ é uma função contínua. Defina $g: B \longrightarrow G$ por $g(b)=f(b, e)$. Note que $h(b, s)=h(b, e s)=h(b, e) s=(b, f(b, e)) s=(b, g(b)) s=(b, g(b) s)$. 
Proposição 2.2.12. Sejam $\xi=(X, p, B, G)$ um $G$-fibrado principal e $f: B_{1} \longrightarrow B$ uma função contínua. Existe um homeomorfismo $g: X_{1} \bmod G \longrightarrow B_{1}$, no qual $X_{1} \subset B_{1} \times X$ é o conjunto dos pares $\left(b_{1}, x\right)$ tais que $f\left(b_{1}\right)=g(x)$, que faz o seguinte diagrama comutar.

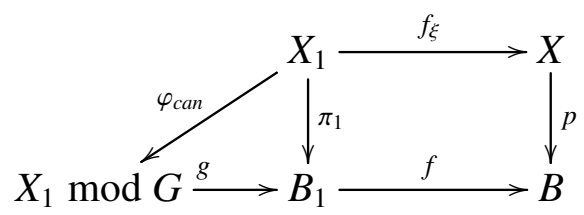

A função $f_{\xi}: X_{1} \longrightarrow X$ é a projeção na segunda coordenada. Então, $f^{*}(\xi)=\left(X_{1}, \pi_{1}, B_{1}, G\right)$ é um G-fibrado principal (chamado G-fibrado principal induzido de $\xi$ por $f$ ) e $\left(f_{\xi}, f\right)$ é um morfismo entre G-fibrados principais.

Demonstração: O espaço $X_{1}$ se torna um $G$-espaço com a ação livre $X_{1} \times G \longrightarrow X_{1},\left(b_{1}, x\right) g=$ $\left(b_{1}, x g\right)$. Defina $g\left(\left(b_{1}, x\right) G\right)=b_{1}$. É fácil verificar que $g$ é um homeomorfismo e que o diagrama acima comuta. Também, $\tau^{*}: X_{1}^{*} \longrightarrow G$, dada por $\tau^{*}\left(\left(b_{1}, x\right),\left(b_{1}, x g\right)\right)=g=\tau(x, x g)$, é contínua. Portanto, $f^{*}(\xi)$ é um $G$-fibrado principal. Note que $f_{\xi}\left(\left(b_{1}, x\right) g\right)=f_{\xi}\left(\left(b_{1}, x g\right)\right)=x g=f_{\xi}\left(b_{1}, x\right) g$. Logo, $f_{\xi}$ é $G$-equivariante e $\left(f_{\xi}, f\right)$ é um morfismo.

OBS 2.2.13. Dados um subespaço $A \subset B$ e a aplicação inclusão $i: A \hookrightarrow B$, denotaremos $o$ G-fibrado principal sobre $A, i^{*}(A)$, por $\left.\xi\right|_{A}$.

OBS 2.2.14. Como no caso de fibrados vetoriais, se $(v, f): \xi \longrightarrow \eta$ for um morfismo entre $G$-fibrados principais, podemos fatorá-lo em $\eta \stackrel{g}{\longrightarrow} f^{*}(\xi) \stackrel{f_{\xi}}{\longrightarrow} \xi$, no qual $g(y)=\left(p_{\eta}(y), v(y)\right)$. Então, $\eta$ e $f^{*}(\xi)$ são $B(\eta)$-isomorfos como G-fibrados principais. Note ainda que $f^{*}: \mathbf{P B}_{\mathbf{B}} \longrightarrow$ $\mathbf{P B}_{\mathbf{B}_{1}}$ é um funtor covariante.

Definição 2.2.15. Sejam $\xi$ e $\eta$ dois G-fibrados principais sobre B. Então, $\xi$ e $\eta$ são localmente isomorfos se, para cada $b \in B$, existe uma vizinhança aberta $U \subset B$ de $b$, tal que $\left.\xi\right|_{U}$ e $\left.\eta\right|_{U}$ são U-isomorfos, como G-fibrados principais. Diz-se que $\xi$ é trivial ou localmente trivial se for isomorfo ou localmente isomorfo ao G-fibrado principal trivial (Exemplo 2.2.4). 


\subsection{Fibrados}

Seja $\xi=(X, p, B, G)$ um $G$-fibrado principal. Dado $F$ um $G$-espaço à esquerda, pode-se dar a $X \times F$ uma estrutura de $G$-espaço (à direita) da seguinte maneira; $(x, y) s=\left(x s, s^{-1} y\right)$, para todo $(x, y) \in X \times F$ e $s \in G$. O conjunto de órbitas de $X \times F$ será denotado por $X_{F}$. Seja $p_{F}: X_{F} \longrightarrow B$ definida por $p_{F}((x, y) G)=p(x)$, para cada $(x, y) \in X \times F$.

Definição 2.3.1. A quíntupla $\xi[F]=\left(X_{F}, p_{F}, B, G, F\right)$ é chamada fibrado associado ao $G$ fibrado principal $\xi$ com espaço base B e fibra $F$. O grupo topológico $G$ é chamado de grupo estrutural do fibrado $\xi[F]$. Às vezes, $p_{F}$ será denotado também por $p_{\xi[F]}$.

Proposição 2.3.2. Para cada $b \in B, p_{F}^{-1}(b)$ é homeomorfo a $F$.

Demonstração: Seja $x_{0} \in X$ tal que $p\left(x_{0}\right)=b$. Defina $f: F \longrightarrow X_{F}$ por $f(y)=\left(x_{0}, y\right) G$. Tem-se $p_{F}\left(\left(x_{0}, y\right) G\right)=p\left(x_{0}\right)=b$, então pode-se considerar $f: F \longrightarrow p_{F}^{-1}(b)$. Seja $g_{1}$ : $p^{-1}(b) \times F \longrightarrow F$, dada por $g_{1}(x, y)=\tau\left(x_{0}, x\right) y$, no qual $\tau$ é a função translação de $\xi$. Como $g_{1}(x, y)=g_{1}((x, y) s)$, pode-se induzir $g: p_{F}^{-1}(b) \longrightarrow F$. Então,

$$
\begin{aligned}
& g \circ f(y)=g\left(\left(x_{0}, y\right) G\right)=g_{1}\left(x_{0}, y\right)=\tau\left(x_{0}, x_{0}\right) y=y \mathrm{e} \\
& f \circ g((x, y) G)=f\left(\tau\left(x_{0}, x\right) y\right)=\left(x_{0}, \tau\left(x_{0}, x\right) y\right) G=\left(x \tau\left(x_{0}, x\right), \tau\left(x_{0}, x\right) y\right) G=(x, y) G .
\end{aligned}
$$

Logo, $p_{F}^{-1}(b)$ é homeomorfo a $F$.

OBS 2.3.3. Note que se $F=G$, então $X_{G}$ e $X$ são homeomorfos. De fato, $X_{G} \longrightarrow X$, dada por $(x, g) G \mapsto x g$, define um homeomorfismo.

Seja $(u, f): \xi \longrightarrow \eta$ um morfismo entre $G$-fibrados principais. Considere

$$
u \times I d_{F}: X(\xi) \times F \longrightarrow X(\eta) \times F
$$

Passando ao quociente, obtém-se $u_{F}: X(\xi)_{F} \longrightarrow X(\eta)_{F}$, dada por $u_{F}((x, y) G)=(u(x), y) G$. O diagrama abaixo é comutativo.

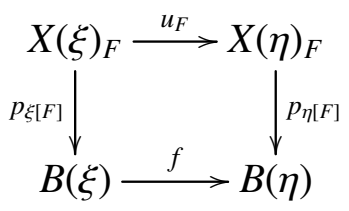


Definição 2.3.4. Um morfismo entre $\xi[F]$ e $\eta[F]$ é um par de funções da forma $\left(u_{F}, f\right)$, no qual $(u, f)$ é um morfismo entre $\xi$ e $\eta$.

OBS 2.3.5. As definições de B-morfismo e B-isomorfismo entre fibrados são inteiramente análogas às anteriores.

Exemplo 2.3.6 (Fibrado trivial).

Sejam $\xi=\left(B \times G, \pi_{1}, B, G\right)$ um $G$-fibrado principal trivial e $F$ um $G$-espaço à esquerda. Então, $(B \times G)_{F}$ é homeomorfo a $B \times F$. De fato: defina $h:(B \times G)_{F} \longrightarrow B \times F$ por $\left.h((b, g), y) G\right)=$ $(b, g y) e h^{\prime}: B \times F \longrightarrow(B \times G)_{F}$ por $h^{\prime}(b, y)=((b, e) y) G$. Então, $h \circ h^{\prime}(b, y)=h(((b, e) y) G)=$ $(b, y) e h^{\prime} \circ h(((b, g) y) G)=h^{\prime}(b, g y)=((b, e) g y) G=((b, e) g y) g^{-1} G=((b, g) y) G$. Mostra-se que $h$ e $h^{\prime}$ são contínuas e estão bem definidas. Portanto, hé um homeomorfismo. Então, o fibrado associado do G-fibrado principal trivial é da forma $(B \times F, \pi, B, G, F)$.

Definição 2.3.7. Dois fibrados $\xi[F]$ e $\eta[F]$ são localmente isomorfos quando seus $G$-fibrados principais $\xi$ e $\eta$ são localmente isomorfos. Também, $\xi[F]$ é trivial ou localmente trivial se $\xi$ for trivial (Exemplo 2.2.4) ou localmente trivial, respectivamente.

Teorema 2.3.8. Sejam $F$ um $G$-espaço à esquerda e $\xi[F]=\left(B \times F, \pi_{1}, B, G, F\right)$ o fibrado trivial. Os B-isomorfismos $\xi[F] \rightarrow \xi[F]$ são da forma $h_{g}(b, y)=(b, g(b) y)$, no qual $g: B \longrightarrow G$ é uma função contínua.

Demonstração: Seja $u: B \times G \longrightarrow B \times G$ um $B$-isomorfismo. Então, do Teorema 2.2.11, temos $u(b, s)=(b, g(b) s)$. Passando ao quociente, obtém-se $u_{F}(((b, s), y) G)=(u(b, s), y) G$, que define $u_{F}^{\prime}: B \times F \longrightarrow B \times F$, no qual $u_{F}^{\prime}(b, y)=(b, g(b) y)$.

Proposição 2.3.9. [19. Capítulo 4, Proposição 6.3, p. 45]. Seja $\xi$ um G-fibrado principal e $\xi[F]$ o fibrado associado de $\xi$ com fibra $F$. Dada uma função contínua $f: B_{1} \longrightarrow B$, existe um isomorfismo entre $f^{*}(\xi[F]$ ) (o $G$-fibrado principal induzido do fibrado associado $\xi[F]$ por $f$ ) $e$ $f^{*}(\xi)[F]$ (o fibrado associado do $G$-fibrado principal induzido de $\xi$ por $f ; f^{*}(\xi)$ ).

\subsection{Fibrados Coordenados}

Nesta seção, veremos como os fibrados vetoriais estão relacionados com os fibrados. 
Definição 2.4.1. Sejam $\xi[F]$ um fibrado ${ }^{1}$ sobre $B$ e $U \subset B$ aberto. Uma carta de $\xi[F]$ sobre $U$ é um isomorfismo $h: U \times F \longrightarrow p_{\xi[F]}^{-1}(U)$. Um atlas para um fibrado $\xi[F]$ é uma coleção $\left\{\left(h_{i}, V_{i}\right)\right\}$, com $i \in I$, no qual $h_{i}: V_{i} \times F \longrightarrow p_{\xi[F]}^{-1}\left(V_{i}\right)$ é uma carta sobre $V_{i} e \bigcup_{i \in I} V_{i}=B$. Neste caso, dizemos que $\xi[F]$ é um fibrado coordenado.

OBS 2.4.2. Um fibrado é localmente trivial se ele possui um atlas.

Proposição 2.4.3. Sejam $h_{1}, h_{2}: U \times F \longrightarrow p_{\xi[F]}^{-1}(U)$ duas cartas. Então, existe uma única função contínua $g: U \longrightarrow G$ tal que $h_{1}(b, y)=h_{2}(b, g(b) y)$, para cada $(b, y) \in U \times F$.

Demonstração: Basta notar que $h_{2}^{-1} \circ h_{1}: U \times F \longrightarrow U \times F$ é um $B$-isomorfismo entre fibrados triviais.

OBS 2.4.4. Note que no caso em que $h_{1}$ e $h_{2}$ são cartas entre fibrados vetoriais, gé uma função entre $U$ e $G L(n, \mathbb{R})$.

Definição 2.4.5. Um sistema de funções transições relativo a uma cobertura abertd $\left.\right|^{2}\left\{V_{i}\right\}_{i \in I}$ de um espaço B é uma família de funções contínuas $g_{i j}: V_{i} \cap V_{j} \longrightarrow G$ que satisfazem a seguinte propriedade: $g_{i k}(b)=g_{i j}(b) g_{j k}(b)$, para cada $i, j, k \in I$ e $b \in V_{i} \cap V_{j} \cap V_{k}$.

OBS 2.4.6. Da propriedade acima, tem-se $g_{i i}(b)=e \quad$ e $g_{j i}(b)=g_{i j}^{-1}(b)$.

Proposição 2.4.7. Todo fibrado localmente trivial possui um sistema de funções transições.

Demonstração: Seja $\xi[F]$ um fibrado localmente trivial com espaço base $B$ e atlas $\left\{\left(h_{i}, V_{i}\right)\right\}$. Existe $g_{i j}: V_{i} \cap V_{j} \longrightarrow G$ definida por $h_{i}^{-1} \circ h_{j}(b, y)=\left(b, g_{i j}(b) y\right)$, para todo $b \in V_{i} \cap V_{j}$ e $y \in F$. Note que dado $b \in V_{i} \cap V_{j} \cap V_{k}$,

$$
h_{i}^{-1} \circ h_{k}(b, y)=\left(b, g_{i k}(b) y\right)=h_{i}^{-1} \circ h_{j} \circ h_{j}^{-1} \circ h_{k}(b, y)=\left(b, g_{i j}(b) g_{j k}(b) y\right) \text {. }
$$

Da unicidade, $g_{i k}(b)=g_{i j}(b) g_{j k}(b)$.

Definição 2.4.8. Dois sistemas de funções transições $\left\{g_{i j}\right\}$ e $\left\{g_{i j}^{\prime}\right\}$ de uma mesma cobertura $\left\{V_{i}\right\}$ de $B$ são equivalentes se, e somente se, existem funções contínuas $r_{i}: V_{i} \longrightarrow G$ tais que $g^{\prime}{ }_{i j}(b)=r_{i}^{-1}(b) g_{i j}(b) r_{j}(b)$, para todo $b \in V_{i} \cap V_{j}$.

\footnotetext{
${ }^{1} \xi[F]$ é um fibrado associado a algum $G$-fibrado principal $\xi$.

${ }^{2}$ Vide Definição 6.6.1
} 
Para verificar que dois fibrados, com mesmo grupo sobre um mesmo espaço base, com atlas sobre a mesma cobertura são isomorfos basta olhar para as funções transições, como mostra o teorema a seguir.

Teorema 2.4.9. [19. Capítulo 5, Teorema 2.7, p. 61]. Sejam $\eta[F], \eta^{\prime}[F]$ dois fibrados com fibra $F$ e grupo estrutural $G$ sobre $B$, com atlas $\left\{V_{i}, h_{i}\right\},\left\{V_{i}, h_{i}^{\prime}\right\}$ e funções transições $\left\{g_{i j}\right\},\left\{g^{\prime}{ }_{i j}\right\}$, respectivamente. Então, $\eta[F]$ e $\eta^{\prime}[F]$ são B-isomorfos se, e somente se, $\left\{g_{i j}\right\}$ e $\left\{g^{\prime}{ }_{i j}\right\}$ são equivalentes.

As funções transições nos permitem construir fibrados localmente triviais, cujo sistema de funções transições coincide com o já dado.

Teorema 2.4.10. [19. Capítulo 5, Teorema 3.2, p. 62]. Sejam $\left\{V_{i}\right\}$, com $i \in I$, uma cobertura aberta de B, G um grupo topológico, $F$ um $G$-espaço à esquerda e $\left\{g_{i j}\right\}$ um sistema de funções transições associado à cobertura aberta $\left\{V_{i}\right\}$. Então, existem um fibrado $\eta=\xi[F]$ e um atlas $\left\{V_{i}, h_{i}\right\}$ para $\eta$ tal que as funções transições sejam $\left\{g_{i j}\right\}$. Mais ainda, $\eta$ é único a menos de B-isomorfismos.

OBS 2.4.11. No caso em que $F=\mathbb{R}^{n}$ e $G=G L(n, \mathbb{R}), \eta$ admite uma estrutura de fibrado vetorial. As classes de isomorfismos de fibrados vetoriais $k$-dimensionais com fibra $\mathbb{R}^{k}$ e grupo $G L(k, \mathbb{R})$ são determinadas por funções transições que tomam valores em $G L(k, \mathbb{R})$. Existe uma bijeção entre as classes de isomorfismos entre fibrados com fibra $\mathbb{R}^{k}$ e grupo $G L(k, \mathbb{R})$ e as classes de isomorfismos entre fibrados vetoriais $k$-dimensionais.

OBS 2.4.12. Dado um fibrado vetorial $\xi^{n}$, o fibrado associado em esfera $S(\xi)$ é um fibrado coordenado cuja fibra é $S^{n}$ e, pelo Teorema 1.6.1 as funções transições tomam valores em $O(n)$. De fato, pois $\langle x, y\rangle=\beta\left(h_{j}^{*}(b, x), h_{j}^{*}(b, y)\right)=\beta\left(h_{i}^{*}\left(b, g_{i j}(b) x\right), h_{i}^{*}\left(b, g_{i j}(b) y\right)\right)=\left\langle g_{i j}(b) x, g_{i j}(b) y\right\rangle$. O fibrado projetivo associado $P(\xi)$ também é um exemplo de fibrado coordenado, no qual a fibra é $\mathbb{R} P^{n-1}$. 


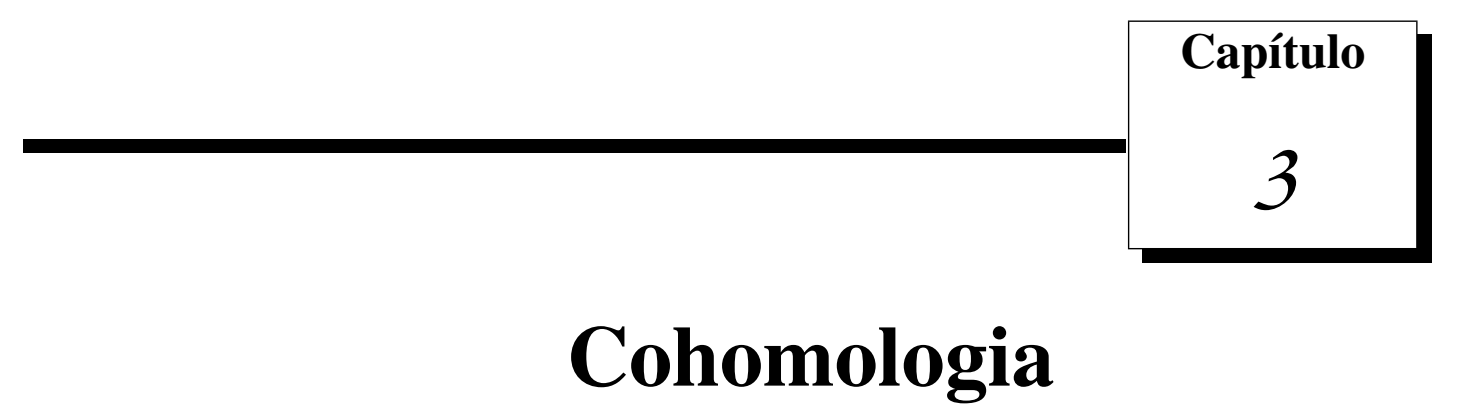

Nesse capítulo, apresentaremos a construção dos $R$-módulos de cohomologia de Čech relativos, no qual $R$ é um anel comutativo com unidade. Para tanto, é necessária a descrição de alguns conceitos básicos tais como sistemas diretos, limite direto de $R$-módulos, os $R$-módulos de cohomologia simplicial relativo e o nervo de uma cobertura, como veremos nas seções 3.1 . 3.2, 3.3 e 3.4, respectivamente. Na Seção 3.5, definimos os $R$-módulos de cohomologia de Čech. Usando o isomorfismo entre a cohomologia de Alexander [Capítulo 6, Seção 6.4] e a cohomologia de Čech, definimos a sequência de Mayer-Vietoris e produto cup com abertos para a cohomologia de Čech. As referências básicas para este capítulo são: o artigo Čech cohomology theory and the axioms de C.H. Dowker [10] e os livros Algebraic Topology: Homology and Cohomology de A.H. Wallace [38], Foundations of Algebraic Topology de S. Eilenberg e N. Steenrod [13], Algebraic Topology de E. Spanier [33] e Elements of Algebraic Topology de J.R. Munkres [29].

\subsection{Sistemas Diretos}

Definição 3.1.1. Um conjunto $M$ com uma relação $\leq$ de quase-ordem (isto é, uma relação reflexiva e transitiva) é chamado conjunto dinigido se, para cada par $\alpha, \beta \in M$, existe $\gamma \in M$ tal que $\alpha \leq \gamma$ e $\beta \leq \gamma$. Também usaremos a notação $\gamma \geq \alpha$, $\beta$. Um subconjunto dirigido $M^{\prime}$ de $M e ́$ um subconjunto $M^{\prime} \subset M$, no qual $M^{\prime}$ é um conjunto dirigido com relação a $\leq$. Um subconjunto 
dirigido $M^{\prime}$ de $M$ é cofinal em $M$ se, dado $\alpha \in M$, existe $\beta \in M^{\prime}$ tal que $\alpha \leq \beta$ em $M$. Se $M$ e $N$ são dois conjuntos dirigidos, uma função $\phi: M \longrightarrow N$ é chamada aplicação entre conjuntos dirigidos se ela preserva ordem, isto é, se $\alpha \leq \beta$, então $\phi(\alpha) \leq \phi(\beta)$.

OBS 3.1.2. Note que a condição para $M^{\prime}$ ser cofinal em $M$ garante que $M^{\prime}$ seja um subconjunto dirigido. Um conjunto dirigido $M$ é uma categoria cujo os objetos são seus elementos e seus morfismos são as relações $\alpha \leq \beta$ em $M(\alpha \rightarrow \beta)$.

Definição 3.1.3. Um sistema direto de conjuntos $\{X, \pi\}$ sobre um conjunto dirigido $M$ é uma função que associa cada $\alpha \in M$ a um conjunto $X^{\alpha}$ e cada par $\alpha \leq \beta$ em $M$ a uma função $\pi_{\beta}^{\alpha}: X^{\alpha} \longrightarrow X^{\beta}$, que satisfaz as seguintes relações:

- $\pi_{\alpha}^{\alpha}=I d_{X^{\alpha}}$

- Se $\alpha \leq \beta \leq \gamma$, então $\pi_{\gamma}^{\beta} \circ \pi_{\beta}^{\alpha}=\pi_{\gamma}^{\alpha}$.

As funções $\pi$ são chamadas projeções.

OBS 3.1.4. Um sistema direto sobre um conjunto dirigido $M$ é um funtor covariante.

Definição 3.1.5. Sejam $\{X, \pi\} e\left\{X^{\prime}, \pi^{\prime}\right\}$ dois sistemas diretos sobre os conjuntos dirigidos $M e$ $M^{\prime}$, respectivamente. Uma aplicação entre os sistemas dinetos $\{X, \pi\}$ e $\left\{X^{\prime}, \pi^{\prime}\right\}$, denotada por $\Phi:\{X, \pi\} \longrightarrow\left\{X^{\prime}, \pi^{\prime}\right\}$, consiste de uma aplicação entre conjuntos dirigidos $\phi: M \longrightarrow M^{\prime} e$ de funções $\phi^{\alpha}: X^{\alpha} \longrightarrow X^{\prime \phi(\alpha)}$, com $\alpha \in M$, tais que se $\alpha \leq \beta$ em $M$, fazem o diagrama abaixo comutar.

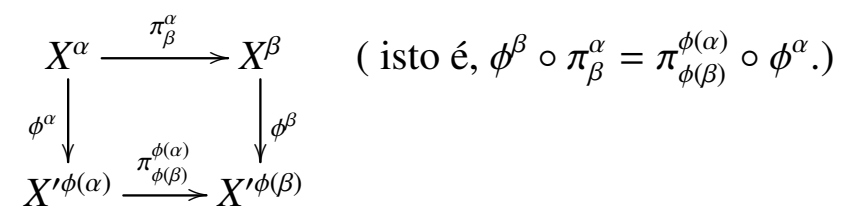

OBS 3.1.6. Sistemas diretos e suas aplicações formam uma categoria.

Definição 3.1.7. Seja $M^{\prime}$ um subconjunto dirigido do conjunto dirigido M. Dado um sistema direto $\{X, \pi\}$ sobre $M$, os conjuntos e funções de $\{X, \pi\}$ que correspondem a conjuntos e relações indexadas em $M^{\prime}$, formam um sistema direto $\left\{X^{\prime}, \pi^{\prime}\right\}$ sobre $M^{\prime}$ chamado subsistema dineto de $\{X, \pi\}$ sobre $M^{\prime}$. Se $M^{\prime}$ é cofinal em $M$, então o subsistema será chamado de subsistema cofinal 
direto de $\{X, \pi\}$ sobre $M^{\prime}$. A aplicação inclusão $\phi: M^{\prime} \longrightarrow M$ e as aplicações identidades $\phi^{\alpha}: X^{\prime \alpha} \longrightarrow X^{\alpha}$, com $\alpha \in M^{\prime}$, formam uma aplicação $\Phi:\left\{X^{\prime}, \pi^{\prime}\right\} \hookrightarrow\{X, \pi\}$ entre sistemas diretos, chamada de injeção do subsistema $\left\{X^{\prime}, \pi^{\prime}\right\}$ no sistema $\{X, \pi\}$.

OBS 3.1.8. Se $M^{\prime}$ for um único ponto $\alpha$, então o subsistema $\left\{X^{\prime}, \pi^{\prime}\right\}$ se reduz a um conjunto $X^{\prime}$ com a aplicação identidade. Podemos definir uma aplicação $\Phi:\{X, \pi\} \longrightarrow X^{\prime}$, no qual $\phi: M \longrightarrow M^{\prime}$ é a aplicação constante e para cada $\alpha \in M, \phi^{\alpha}: X^{\alpha} \longrightarrow X^{\prime}$ é tal que se $\alpha \leq \beta$, $\phi^{\beta} \circ \pi_{\beta}^{\alpha}=\phi^{\alpha}$.

\subsection{Limites Diretos}

Sejam $R$ um anel comutativo com unidade e $\{G, \pi\}$ um sistema direto sobre um conjunto dirigido $M$, no qual cada $G^{\alpha}$ é um $R$-módulo e cada $\pi_{\beta}^{\alpha}$ é um $R$-homomorfismo. Seja $i_{\alpha}: G^{\alpha} \longrightarrow$ $\bigoplus_{\alpha \in M} G^{\alpha}$ a inclusão. Denotaremos um elemento $i_{\alpha}(g)$ em $\bigoplus_{\alpha \in M} G^{\alpha}$ simplesmente por $g$.

Definição 3.2.1. Para cada $\alpha \leq \beta$ em $M$ e cada $g^{\alpha} \in G^{\alpha}$, o elemento $\pi_{\beta}^{\alpha}\left(g^{\alpha}\right)-g^{\alpha}$ em $\bigoplus_{\alpha \in M} G^{\alpha} e^{\prime}$ chamado uma relação. Seja $Q$ o submódulo de $\bigoplus_{\alpha \in M} G^{\alpha}$ gerado por todas as relações. O limite direto de $\{G, \pi\}$ é o $R$-módulo $G^{\infty}=\frac{\bigoplus_{\alpha \in M} G^{\alpha}}{Q}$.

A projeção canônica $\pi_{c a n}: \bigoplus_{\alpha \in M} G^{\alpha} \longrightarrow G^{\infty}$ induz $R$-homomorfismos chamados projeções $\pi_{\alpha}=\pi_{c a n} \circ i_{\alpha}: G^{\alpha} \longrightarrow G^{\infty}$.

Lema 3.2.2. Se $\alpha \leq \beta$, então $\pi_{\beta} \circ \pi_{\beta}^{\alpha}=\pi_{\alpha}$.

Demonstração: Como $\pi_{\beta} \circ \pi_{\beta}^{\alpha}\left(g^{\alpha}\right)=\pi_{\beta}^{\alpha}\left(g^{\alpha}\right)+Q$ e $\pi_{\alpha}\left(g^{\alpha}\right)=g^{\alpha}+Q$, segue que $\pi_{\beta} \circ \pi_{\beta}^{\alpha}\left(g^{\alpha}\right)-$ $\pi_{\alpha}\left(g^{\alpha}\right)=\left(\pi_{\beta}^{\alpha}\left(g^{\alpha}\right)-g^{\alpha}\right)+Q=Q$.

Lema 3.2.3. Se $u \in G^{\infty}$, então existe $\alpha$ e $g^{\alpha} \in G^{\alpha}$ tal que $\pi_{\alpha}\left(g^{\alpha}\right)=u$.

Demonstração: Suponhamos que $u=v+Q$, no qual $v=\sum_{k=1}^{n} g^{\alpha_{k}}$. Sejam $\alpha \in M$ tal que $\alpha \geq \alpha_{k}$ $(1 \leq k \leq n)$ e $v^{\prime}=\sum_{k=1}^{n} g^{k} \in G^{\alpha}$, no qual $g^{k}=\pi_{\alpha}^{\alpha_{k}}\left(g^{\alpha_{k}}\right)$. Então, $v-v^{\prime}=\sum_{k=1}^{n} g^{\alpha_{k}}-g^{k} \in Q$. Logo $u=v+Q=v^{\prime}+Q=\pi_{\alpha}\left(v^{\prime}\right)$. 
Lema 3.2.4. Se $g \in G^{\gamma}$ e $\pi_{\gamma}(g)=Q$, então existe um $\delta \geq \gamma$ tal que $\pi_{\delta}^{\gamma}(g)=0$ (elemento neutro do grupo).

Demonstração: Por hipótese, $g=\sum_{k=1}^{n} \pi_{\beta_{k}}^{\alpha_{k}}\left(g^{\alpha_{k}}\right)-g^{\alpha_{k}}$. Sejam $\delta \geq \gamma, \beta_{i}, \alpha_{j}$ em $M$. Podemos escrever $\pi_{\delta}^{\gamma}(g)=\left(\pi_{\delta}^{\gamma}(g)-g\right)+g$ e $\pi_{\beta_{k}}^{\alpha_{k}}\left(g^{\alpha_{k}}\right)-g^{\alpha_{k}}=\pi_{\delta}^{\alpha_{k}}\left(g^{\alpha_{k}}\right)-g^{\alpha_{k}}+\pi_{\delta}^{\alpha_{k}}\left(-\pi_{\beta_{k}}^{\alpha_{k}}\left(g^{\alpha_{k}}\right)\right)-\pi_{\beta_{k}}^{\alpha_{k}}\left(g^{\alpha_{k}}\right)$. Logo, podemos escrever $\pi_{\delta}^{\gamma}(g)=\sum_{\alpha} \pi_{\delta}^{\alpha}\left(g^{\alpha}\right)-g^{\alpha}$. Analisando coordenada a coordenada na igualdade anterior, verificamos o resultado.

Teorema 3.2.5. Se $\{G, \pi\}$ é um sistema direto sobre $M$ tal que para cada $\alpha \leq \beta$ em $M$, $\pi_{\beta}^{\alpha}$ é injetor, então $\pi_{\alpha}: G^{\alpha} \longrightarrow G^{\infty}$ é injetora.

Demonstração: A demonstração segue imediatamente do Lema 3.2.4.

Lema 3.2.6. Existe $\gamma \geq \alpha, \beta$ tal que $\pi_{\gamma}^{\alpha}\left(g^{\alpha}\right)=\pi_{\gamma}^{\beta}\left(g^{\beta}\right)$ se, e somente se, $\pi_{\alpha}\left(g^{\alpha}\right)=\pi_{\beta}\left(g^{\beta}\right)$.

Demonstração: Se existe $\gamma \geq \alpha, \beta$ tal que $\pi_{\gamma}^{\alpha}\left(g^{\alpha}\right)=\pi_{\gamma}^{\beta}\left(g^{\beta}\right)$, então

$$
\left(\left(\pi_{\gamma}^{\alpha}\left(g^{\alpha}\right)-g^{\alpha}\right)-\left(\pi_{\gamma}^{\beta}\left(g^{\beta}\right)-g^{\beta}\right)\right)=g^{\beta}-g^{\alpha}=\pi_{\beta}\left(g^{\beta}\right)-\pi_{\alpha}\left(g^{\alpha}\right) \in Q .
$$

Agora, se $\pi_{\alpha}\left(g^{\alpha}\right)=\pi_{\beta}\left(g^{\beta}\right)$, tome $\delta \geq \alpha, \beta$ e seja $g=\pi_{\delta}^{\alpha}\left(g^{\alpha}\right)-\pi_{\delta}^{\beta}\left(g^{\beta}\right)$. Então, $\pi_{\delta}(g)=Q$. Logo existe $\gamma \geq \delta$ tal que $\pi_{\delta}^{\gamma}(g)=\pi_{\gamma}^{\alpha}\left(g^{\alpha}\right)-\pi_{\gamma}^{\beta}\left(g^{\beta}\right)=0$.

Dizemos que dois elementos $g^{\alpha} \in G^{\alpha}$ e $g^{\beta} \in G^{\beta}$ são equivalentes, se existe $\gamma \geq \alpha, \beta$ tal que $\pi_{\gamma}^{\alpha}\left(g^{\alpha}\right)=\pi_{\gamma}^{\beta}\left(g^{\beta}\right)$. Esta relação nos dá uma relação de equivalência. Seja $6 \mathfrak{5}$ o conjunto de todas as classes desta relação. Podemos somar duas classes $\left[g_{1}\right],\left[g_{2}\right]$, escolhendo representantes $\left[g_{1}^{\alpha}\right],\left[g_{2}^{\alpha}\right]$ com mesmo índice e definindo $\left[g_{1}\right]+\left[g_{2}\right]=\left[g_{1}^{\alpha}+g_{2}^{\alpha}\right]$. Também definimos $r\left[g^{\alpha}\right]$ como sendo a classe $\left[r g^{\alpha}\right]$, para $r \in R$. Então, $\mathfrak{b}^{2}$ é um $R$-módulo. A aplicação $\mathfrak{5} \longrightarrow G^{\infty}$ que leva $\left[g^{\alpha}\right] \mapsto g^{\alpha}+Q$ é um $R$-isomorfismo.

Definição 3.2.7. Seja $\Phi:\{G, \pi\} \longrightarrow\left\{G^{\prime}, \pi^{\prime}\right\}$ uma aplicação entre dois sistemas diretos de $R$ módulos sobre os conjunto dirigidos $M, M^{\prime}$, respectivamente. Os $R$-homomorfismos $\phi^{\alpha}: G^{\alpha} \longrightarrow$ $G^{\prime \phi(\alpha)}$ definem um R-homomorfismo $\widetilde{\Phi}: \bigoplus_{\alpha \in M} G^{\alpha} \longrightarrow \bigoplus_{\alpha^{\prime} \in M^{\prime}} G^{\alpha^{\prime}}$, dado por $\sum g^{\alpha} \mapsto \sum \phi^{\alpha}\left(g^{\alpha}\right)$.

OBS 3.2.8. Note que $\widetilde{\Phi}\left(\pi_{\beta}^{\alpha}\left(g^{\alpha}\right)-g^{\alpha}\right)={\pi^{\prime}}_{\phi(\beta)}^{\phi(\alpha)}\left(\phi^{\alpha}\left(g^{\alpha}\right)\right)-\phi^{\alpha}\left(g^{\alpha}\right)$. 
Definição 3.2.9. O R-homomorfismo $\widetilde{\Phi}$ induz um $R$-homomorfismo $\Phi^{\infty}: G^{\infty} \longrightarrow G^{\prime \infty}$ chamado $o$ limite direto de $\Phi$.

OBS 3.2.10. $O$ R-homomorfismo $\Phi^{\infty}: G^{\infty} \longrightarrow G^{\prime \infty}$ torna o seguinte diagrama comutativo.

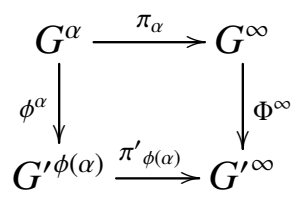

Teorema 3.2.11. Seja $\{G, \pi\}$ um sistema direto de R-módulos sobre um conjunto dirigido $M$ e seja $M^{\prime} \subset M$ um subconjunto cofinal. Então, a injeção do subsistema cofinal $\left\{G^{\prime}, \pi^{\prime}\right\}$ em $M^{\prime}$ no sistema $\{G, \pi\}$ induz um isomorfismo.

Demonstração: Seja $\Phi^{\infty}: G^{\prime \infty} \longrightarrow G^{\infty}$ o limite direto da injeção $\Phi:\left\{X^{\prime}, \pi^{\prime}\right\} \longrightarrow\{X, \pi\}$. Dado $g^{\alpha}+Q \in G^{\infty}$, tome $\beta \in M^{\prime}$ tal que $\alpha \leq \beta$ e defina $g^{\beta}=\pi_{\beta}^{\alpha}\left(g^{\alpha}\right)$. Então, $\Phi^{\infty}\left(g^{\beta}+Q^{\prime}\right)=g^{\beta}+Q=$ $g^{\alpha}+\left(g^{\beta}-\pi_{\beta}^{\alpha}\left(g^{\alpha}\right)\right)+Q=g^{\alpha}+Q$. Assim, $\Phi^{\infty}$ é sobrejetora.

Agora, se $\Phi^{\infty}\left(g^{\alpha}+Q^{\prime}\right)=g^{\alpha}+Q=Q$, então existe $\beta \in M$ tal que $\pi_{\beta}^{\alpha}\left(g^{\alpha}\right)=0$. Existe $\gamma \in M^{\prime}$ tal que $\gamma \geq \beta \geq \alpha$. Então, $\pi_{\gamma}^{\beta} \circ \pi_{\beta}^{\alpha}\left(g^{\alpha}\right)=0=\pi_{\gamma}^{\alpha}\left(g^{\alpha}\right)$. Podemos escrever $g^{\alpha}+Q^{\prime}=$ $\pi_{\gamma}^{\alpha}\left(g^{\alpha}\right)+g^{\alpha}+Q^{\prime}=Q^{\prime}$. Assim, $\Phi^{\infty}$ é injetora. Portanto, $\Phi^{\infty}$ é um isomorfismo.

O limite direto da composição de duas aplicações entre sistemas diretos de $R$-módulos é a composição dos limites diretos das aplicações, como mostra o teorema a seguir.

Teorema 3.2.12. [13. Capítulo 8, Teorema 4.12, p. 223]. Sejam $\{X, \pi\},\left\{Y, \pi^{\prime}\right\}$ e $\left\{Z, \pi^{\prime \prime}\right\}$ sistemas diretos de R-módulos e $\Phi:\{X, \pi\} \longrightarrow\left\{Y, \pi^{\prime}\right\}, \Phi^{\prime}:\left\{Y, \pi^{\prime}\right\} \longrightarrow\left\{Z, \pi^{\prime \prime}\right\}$ aplicações entre os sistemas diretos. Então, $\left(\Phi^{\prime} \circ \Phi\right)^{\infty}=\Phi^{\prime \infty} \circ \Phi^{\infty}$.

\subsection{A cohomologia simplicial}

A teoria de cohomologia simplicial é construída sobre uma categoria especial de conjuntos, a categoria dos pares simpliciais e suas aplicações. Os axiomas de Eilenberg-Steenrod são verificados sem exceção, como pode ser visto em [26], [29] e [33] . 


\subsubsection{Os pares simpliciais}

Os principais conceitos para a construção da categoria dos pares simpliciais são dados a seguir.

Definição 3.3.1. Um complexo simplicial abstrato $K$ é um $\operatorname{par}(V, S)$ no qual $V$ é um conjunto $e$ $S$ é uma coleção de subconjuntos finitos e não vazios de V satisfazendo as seguintes condições:

(i) $\operatorname{Se} v \in V$, então $\{v\} \in S$.

(ii) Se $\tau \subset \sigma \in S$ e se $\tau \neq \emptyset$, então $\tau \in S$.

Os elementos $v \in V$ são chamados vértices e os elementos $\sigma \in S$ são chamados simplexos. Um q-simplexo é um elemento $\sigma \in S$ com $q+1$ vértices, e neste caso, dizemos que dimensão de $\sigma e ́ q$ (isto é, $\operatorname{dim} \sigma=q$ ). Se $\tau \subset \sigma$, $\tau$ é chamado uma face de $\sigma$. Se $\tau$ for um q-simplexo, dizemos que ele é uma q-face de $\sigma$. A dimensão de $K$, denotada por $\operatorname{dim} K$, é definida como sendo -1, se $K$ for vazio e é igual a $n$, se $K$ contém um n-simplexo, mas não contém nenhum $(n+1)$-simplexo. Se $K$ contém $n$-simplexos, para todo $n \geq 0$, dizemos que $\operatorname{dim} K$ é infinita.

OBS 3.3.2. O complexo simplicial $K$ pode ser considerado como sendo igual ao conjunto de seus simplexos. De fato: segue da condição (i) da Definição 3.3.1 que existe uma correspondência bijetora entre os 0-simplexos de $K$ e os vértices de $K$ e, da condição (ii), qualquer simplexo é determinado por suas 0-faces (seus vértices).

Definição 3.3.3. Um subcomplexo $L$ de um complexo simplicial abstrato $K$, o qual será denotado por $L \subset K$, é um par $\left(V^{\prime}, S^{\prime}\right)$ que torna $L$ um complexo simplicial abstrato, no qual $V^{\prime} e ́$ um subconjunto dos vértices de $K$ e cada simplexo de $S^{\prime}$ é um simplexo de K. Chamamos o par $(K, L)$ de par simplicial.

Exemplo 3.3.4. Dado um complexo simplicial abstrato $K$, o seu $q$-dimensional esqueleto $K^{(q)}$ é definido como sendo o subcomplexo consistindo de todos os $p$-simplexos de $K$, para $p \leq q$.

Definição 3.3.5. Sejam $K_{1}$ e $K_{2}$ dois complexos simpliciais abstratos. Uma aplicação simplicial entre $K_{1}$ e $K_{2}$ é uma função $\varphi: K_{1} \longrightarrow K_{2}$, definida por $\varphi(\sigma)=\left\{\varphi^{0}\left(v_{0}\right), \ldots, \varphi^{0}\left(v_{q}\right)\right\} \in K_{2}$, no qual $\sigma=\left\{v_{0}, \ldots, v_{q}\right\} \in K_{1} e \varphi^{0}: K_{1}^{0} \longrightarrow K_{2}^{0}$ é uma função entre os vértices. 
Para qualquer complexo simplicial abstrato $K$, existe uma aplicação simplicial identidade $I d_{K}: K \rightarrow K$. Se $L \subset K$, existe uma inclusão natural $i: L \hookrightarrow K$, a qual é um aplicação simplicial. Dadas aplicações simpliciais $K_{1} \stackrel{\varphi}{\rightarrow} K_{2} \stackrel{\psi}{\rightarrow} K_{3}$, a composição $\psi \circ \varphi: K_{1} \rightarrow K_{3}$ é uma aplicação simplicial, a qual corresponde a uma aplicação vértice composição.

Definição 3.3.6. Duas aplicações simpliciais $f, g: K_{1} \longrightarrow K_{2}$ são chamadas contíguas, quando, para todo simplexo $\sigma \in K_{1}, f(\sigma)$ e $g(\sigma)$ são faces de um mesmo simplexo em $K_{2}$.

Definição 3.3.7. Uma aplicação de pares simpliciais $\varphi:\left(K_{1}, L_{1}\right) \longrightarrow\left(K_{2}, L_{2}\right)$ é uma aplicação simplicial $\varphi: K_{1} \longrightarrow K_{2}$ tal que $\varphi\left(L_{1}\right) \subset L_{2}$, ou seja, $\varphi$ leva cada simplexo de $L_{1}$ em um simplexo de $L_{2}$. Duas aplicações de pares simpliciais $f, g:\left(K_{1}, L_{1}\right) \longrightarrow\left(K_{2}, L_{1}\right)$ são contíguas quando, para todo simplexo $\sigma \in K_{1}, f(\sigma)$ e $g(\sigma)$ são faces de um mesmo simplexo em $K_{2}$ e para todo simplexo $\sigma^{\prime} \in L_{1}, f\left(\sigma^{\prime}\right)$ e $g\left(\sigma^{\prime}\right)$ são faces de um mesmo simplexo em $L_{2}$.

A categoria, cujos objetos são pares simpliciais $(K, L)$ e cujos morfismos são aplicações de pares simpliciais $\varphi:\left(K_{1}, L_{1}\right) \longrightarrow\left(K_{2}, L_{2}\right)$ é chamada categoria dos pares simpliciais.

\subsubsection{Os $R$-módulos de Cohomologia Simplicial Relativos}

Aqui são construídos os $R$-módulos de cohomologia simplicial, no qual $R$ é um anel comutativo com unidade.

Definição 3.3.8. Seja $K$ um complexo simplicial abstrato. Um q-simplexo orientado de $K$ é um q-simplexo $\sigma \in K$ junto com uma classe de equivalência, determinada pelas ordenações dos vértices de $\sigma$. Podemos representar $\sigma$ por meio de seus vértices $\left\{v_{0}, \ldots, v_{q}\right\}$ escrevendo $\sigma=\left\{v_{0}, \ldots, v_{q}\right\}$. Uma ordenação dos vértices de $\sigma$ consiste em uma escolha na ordem dos seus vértices na representação dada na frase anterior. Por exemplo, $\left\{v_{0}, \ldots, v_{q}\right\}$ e $\left\{v_{1}, v_{0}, v_{2}, \ldots, v_{q}\right\}$ são duas ordenações distintas. Dadas duas ordenações $v_{i_{0}}<\ldots<v_{i_{q}}$ e $v_{j_{0}}<\ldots<v_{j_{q}}$ dos vértices de $\sigma$, existe uma permutação $P$ do conjunto $\{0, \ldots, q\}$ tal que $P\left(i_{k}\right)=j_{k}$, para $k=0, \ldots, q$. Definimos uma relação de equivalência sobre o conjunto de todas as ordenações dos vértices de $\sigma$ da seguinte forma: duas ordenações são equivalentes se, e somente se, elas diferem por uma permutação par dos vértices de $\sigma$. Uma escolha de uma classe de equivalência de ordenações dos vértices de $\sigma$ é chamada uma orientação para $\sigma$. Se $v_{0}, \ldots, v_{q}$ são vértices 
de $\sigma$, então $\left[v_{0}, v_{1}, \ldots, v_{q}\right]$ denotará o q-simplexo orientado de $K$ consistindo do simplexo $\sigma$ junto com a classe de equivalência da ordenação $v_{0}<v_{1}<\ldots<v_{q}$ de seus vértices.

Exemplo 3.3.9. Seja $K$ um complexo simplicial abstrato. Se $q<0$, não existem $q$-simplexos orientados. Para todo vértice $v$ de $K$, existe um único 0 -simplexo orientado $[v]$ e, para todo $q$-simplexo de $K$, com $q \geq 1$, correspondem exatamente dois simplexos q-orientados. Assim, se $\sigma$ for um 0-simplexo, existe uma única orientação para $\sigma$. Uma orientação de um 1-simplexo é apenas uma escolha dos vértices inicial e final, a qual pode ser indicada esquematicamente, desenhando-se uma seta ao longo do simplexo. Uma orientação de um 2-simplexo é uma escolha de um sentido preferencial de rotação, a qual pode ser indicada por uma seta circular e, assim, sucessivamente.

Definição 3.3.10. Dado um complexo simplicial abstrato $K$, seja $C_{q}(K ; R)$ o R-módulo geradd ${ }^{1}$ pelos $q$-simplexos orientados de K, chamado o R-módulo de q-cadeias simpliciais de K. Observemos que $C_{q}(K ; R)$ é o $R$-módulo livre cujo posto é igual ao número de q-simplexos orientados de $K$ e que $C_{q}(K ; R)=\{0\}$, para $q<0$ ou $q>\operatorname{dim} K$.

Para $q \geq 1$, definimos um operador bordo $\partial_{q}$ sobre um $q$-simplexo orientado pela fórmula:

$$
\partial_{q}[\sigma]=\partial_{q}\left[v_{0}, v_{1}, \ldots, v_{q}\right]=\sum_{m=0}^{q}(-1)^{m}\left[v_{0}, v_{1}, \ldots, \hat{v}_{m}, \ldots, v_{q}\right]
$$

no qual $\left[v_{0}, v_{1}, \ldots, \hat{v}_{m}, \ldots, v_{q}\right]$ denota o $(q-1)$-simplexo obtido deletando-se o vértice $v_{m}$. Então, $\partial_{q}$ se estende por R-linearidade a um único homomorfismo $\partial_{q}: C_{q}(K ; R) \rightarrow C_{q-1}(K ; R)$ definido pela fórmula

$$
\partial_{q}\left(\sum_{i=1}^{k} r_{i}\left[\sigma_{i}\right]\right)=\sum_{i=1}^{k} r_{i} \partial_{q}\left[\sigma_{i}\right]=\sum_{i=1}^{k} r_{i}\left(\sum_{m=0}^{q}(-1)^{m}\left[v_{0}^{i}, v_{1}^{i}, \ldots, \hat{v}_{m}^{i}, \ldots v_{q}^{i}\right]\right)
$$

Para $q \leq 0$, definimos $\partial_{q}: C_{q}(K ; R) \rightarrow C_{q-1}(K ; R)$ como sendo o homomorfismo trivial.

OBS 3.3.11. $O$ operador bordo $\partial_{q}$ satisfaz $\partial_{q-1} \circ \partial_{q}=0$. Portanto, $C(K ; R)=\left\{C_{q}(K ; R), \partial_{q}\right\}_{q \geq 0}$, é um complexo de cadeias, chamado complexo de cadeias simplicial de K. Observe que dado um par simplicial $(K, L), C_{q}(L ; R)$ é naturalmente um submódulo de $C_{q}(K ; R)$ e $\partial_{q}\left(C_{q}(L ; R)\right) \subset$

\footnotetext{
${ }^{1}$ Vide Definição 6.1 .1
} 
$C_{q-1}(L ; R)$. Assim, $\partial_{q}: C_{q}(L ; R) \rightarrow C_{q-1}(L ; R)$, a restrição do operador bordo, está bem definida.

Definição 3.3.12. O R-módulo quociente $C_{q}(K, L)=\frac{C_{q}(K ; R)}{C_{q}(L ; R)}$ é um $R$-módulo livre, chamado o $R$ módulo de $q$-cadeias simpliciais relativo de $K$ módulo L. Note que $\partial_{q}$ induz um R-homomorfismo $C_{q}(K, L) \rightarrow C_{q-1}(K, L)$, chamado operador bordo relativo, o qual também será denotado por $\partial_{q}$ e satisfaz $\partial_{q-1} \circ \partial_{q}=0$. Dessa forma, obtemos um complexo de cadeias $C(K, L ; R)=$ $\left\{C_{q}(K, L ; R) ; \partial_{q}\right\}_{q \geq 0}=\left\{\frac{C_{q}(K ; R)}{C_{q}(L ; R)} ; \partial_{q}\right\}_{q \geq 0}$.

Definimos o $q$-ésimo $R$-módulo de $q$-cocadeias simpliciais relativo como sendo

$$
C^{q}(K, L ; R)=\operatorname{Hom}\left(C_{q}(K, L) ; R\right)=\operatorname{Hom}\left(\frac{C_{q}(K)}{C_{q}(L)} ; R\right),
$$

no qual Hom $\left(C_{q}(K, L) ; R\right)$ é o conjunto dos $R$-homomorfismos de $C_{q}(K, L)$ em $R$. O operadorcobordo relativo $\delta^{q}: C^{q}(K, L ; R) \rightarrow C^{q+1}(K, L ; R)$ é definido como sendo o dual do operador bordo relativo $\partial_{q}: C_{q}(K, L) \rightarrow C_{q-1}(K, L)$, isto é, $\delta^{q}=\operatorname{Hom}\left(\partial_{\mathrm{q}}, \mathrm{R}\right)$ e, desde que satisfaz $\delta^{q} \circ \delta^{q-1}=0$, obtemos um complexo de cocadeias $\left\{C^{q}(K, L ; R), \delta^{q}\right\}_{q \geq 0}$. O $R$-módulo de cohomologia orientado do par simplicial $(K, L)$, com coeficientes em $R$, também chamado $R$-módulo de cohomologia simplicial é denotado por $H^{q}(K, L ; R)$ e $H^{*}(K, L ; R)=\left\{H^{q}(K, L ; R)\right\}_{q \geq 0}$ é definido como sendo o $R$-módulo de cohomologia graduado do complexo de cocadeias $\left\{C^{q}(K, L ; R), \delta^{q}\right\}_{q \geq 0}$.

OBS 3.3.13. Dada uma aplicação simplicial de pares $f:(K, L) \rightarrow\left(K_{1}, L_{1}\right)$, $f$ induz um $R$ homomorfismo $f^{*}: H^{*}\left(K_{1}, L_{1} ; R\right) \rightarrow H^{*}(K, L ; R)$.

Teorema 3.3.14. [26] Capítulo 3, Corolário 2, p. 108]. Sejam f, $g:\left(K_{1}, L_{1}\right) \longrightarrow\left(K_{2}, L_{2}\right)$ duas aplicações simpliciais contíguas . Então, $f^{*}=g^{*}$.

\subsection{O nervo de uma cobertura}

Uma maneira de associar um par topológico $(X, A)$ a um complexo simplicial abstrato é através do nervo de uma cobertura. Dada uma cobertura aberta para $(X, A)$, identificamos cada aberto como sendo um vértice e criamos um conjunto de simplexos com certas propriedades. $\mathrm{O}$ nervo desta cobertura é, portanto, este complexo simplicial abstrato criado. 
Definição 3.4.1. Uma cobertura aberta de um espaço topológico $(X, \tau)$ é uma função $\alpha: V_{\alpha} \longrightarrow \tau$, que associa cada $v \in V_{\alpha}$ a um aberto $\alpha_{v} \in \tau$ tal que $X=\bigcup_{v \in V_{\alpha}} \alpha_{v}$, no qual $V_{\alpha}$ é um conjunto de índices. Se $A \subset X e V_{\alpha}^{A} \subset V_{\alpha}$ é tal que $A \subset \bigcup_{v \in V_{\alpha}^{A}} \alpha_{v}$, então dizemos que $\alpha=\left(V_{\alpha}, V_{\alpha}^{A}\right)$ é um cobertura aberta do par $(X, A)$. Denotamos o conjunto de todas as coberturas abertas de $X$ por $\operatorname{Cov}(X)$ e o conjunto das coberturas abertas de $(X, A)$ por $\operatorname{Cov}(X, A)$.

Definição 3.4.2. Sejam $\alpha, \beta \in \operatorname{Cov}(X, A)$. Dizemos que $\beta$ é um refinamento de $\alpha$, e denotamos por $\alpha \leq \beta$ quando para qualquer $v \in V_{\beta}$ existe $v^{\prime} \in V_{\alpha}$ tal que $\beta_{v} \subset \alpha_{v^{\prime}}$ e para qualquer $w \in V_{\beta}^{A}$ existe $w^{\prime} \in V_{\alpha}^{A}$ tal que $\beta_{w} \subset \alpha_{w^{\prime}}$.

OBS 3.4.3. Mostra-se que $(\operatorname{Cov}(X, A), \leq)$ é um conjunto dirigido (Vide [13] Capítulo 9, Lema 2.10, p. 235]).

Definição 3.4.4. Sejam $f:(X, A) \longrightarrow(Y, B)$ uma aplicação de pares e $\beta$ uma cobertura aberta de $(Y, B)$. Então, a cobertura $\alpha$ formada pelo mesmo par de índices $\left(V_{\alpha}, V_{\alpha}^{A}\right)=\left(V_{\beta}, V_{\beta}^{B}\right)$ de $\beta$ tal que $\alpha_{v}=f^{-1}\left(\beta_{v}\right)$, para todo $v \in V_{\alpha}$, define uma cobertura aberta para $(X, A)$. Denotaremos $\alpha$ por $f^{-1}(\beta)$.

Definição 3.4.5. Sejam $\alpha$ uma cobertura aberta de $X$ e $\mathcal{S}_{\alpha}$ o complexo simplicial abstrato consistindo de todos os simplexos nos quais os vértices são os elementos de $V_{\alpha}$. O nervo de $\alpha$, denotado por $X_{\alpha}$, é o subcomplexo de $\mathcal{S}_{\alpha}$ consistindo de todos os simplexos s tais que $\bigcap_{v \in s} \alpha_{v} \neq \emptyset$. Se $\alpha$ for uma cobertura aberta do par $(X, A)$, indexada por $\left(V_{\alpha}, V_{\alpha}^{A}\right)$, denotaremos por $A_{\alpha}$ o subcomplexo de $X_{\alpha}$ consistindo de todos os simplexos s com vértices em $V_{\alpha}^{A}$ tais que $A \cap\left(\bigcap_{v \in s} \alpha_{v}\right) \neq \emptyset$. Neste caso, o nervo de $\alpha$ será o par $\left(X_{\alpha}, A_{\alpha}\right)$.

Proposição 3.4.6. $O$ par $\left(X_{\alpha}, A_{\alpha}\right)$ é um par simplicial.

Demonstração: Se $s \in X_{\alpha}$ e $s^{\prime} \subset s$, então $\emptyset \neq \bigcap_{v \in s} \alpha_{v} \subset \bigcap_{v \in s^{\prime}} \alpha_{v}$. Logo $s^{\prime} \in X_{\alpha}$. O caso em que $s \in A_{\alpha}$ é análogo.

Lema 3.4.7. Sejam $f:(X, A) \longrightarrow(Y, B)$ uma aplicação de pares e $\alpha=f^{-1}(\beta)$, no qual $\beta$ é uma cobertura aberta de $(Y, B)$. Então, o nervo $X_{\alpha}$ é um subcomplexo de $Y_{\beta}$ e $A_{\alpha}$ é um subcomplexo de $B_{\beta}$. 
Demonstração: Seja $s \in X_{\alpha}$. Então,

$$
\emptyset \neq \bigcap_{v \in s} \alpha_{v}=\bigcap_{v \in s} f^{-1}\left(\beta_{v}\right)=f^{-1}\left(\bigcap_{v \in s} \beta_{v}\right) .
$$

Logo, $\emptyset \neq \bigcap_{v \in s} \beta_{v}$ e, portanto, $s \in Y_{\beta}$. O caso em que $s \in A_{\alpha}$ é análogo.

OBS 3.4.8. Denotaremos a função inclusão $\left(X_{\alpha}, A_{\alpha}\right) \hookrightarrow\left(Y_{\beta}, B_{\beta}\right)$ por $f_{\beta}$.

Lema 3.4.9. [13. Capítulo 9, Lemas 2.6 e $2.7, \quad$ p. $\quad$ 234]. Seja $f:(X, A) \longrightarrow(X, A)$ a aplicação identidade, então $f_{\alpha}$ será a aplicação identidade, para todo $\alpha \in \operatorname{Cov}(X, A)$. Dadas aplicações de pares $f:(X, A) \longrightarrow(Y, B)$ e $g:(Y, B) \longrightarrow(Z, C)$ e uma cobertura aberta $\gamma \in \operatorname{Cov}(Z, C)$, então $(g \circ f)_{\gamma}=g_{\gamma} \circ f_{\beta}$, no qual $\beta=g^{-1}(\gamma)$.

Definição 3.4.10. Se $\alpha \leq \beta \in \operatorname{Cov}(X, A)$, uma função $p:\left(V_{\beta}, V_{\beta}^{A}\right) \longrightarrow\left(V_{\alpha}, V_{\alpha}^{A}\right)$ é chamada projeção se $\beta_{v} \subset \alpha_{p(v)}$, para cada $v \in V_{\beta}$.

OBS 3.4.11. Note que podemos ter mais de uma função projeção entre $\left(V_{\beta}, V_{\beta}^{A}\right) e\left(V_{\alpha}, V_{\alpha}^{A}\right)$. A função p é uma aplicação de vértices e se estende a uma aplicação simplicial $p:\left(X_{\beta}, A_{\beta}\right) \longrightarrow$ $\left(X_{\alpha}, A_{\alpha}\right), s_{\beta} \mapsto s_{\alpha}$, também chamada projeção.

Proposição 3.4.12. [13. Capítulo 9, Lema 2.15, p. $\quad$ Le 236]. Seja $f:(X, A) \longrightarrow(Y, B)$ uma aplicação de pares. Dados $\alpha, \beta \in \operatorname{Cov}(Y, B)$ tais que $\alpha \leq \beta$, então $\alpha^{\prime}=f^{-1}(\alpha) \leq \beta^{\prime}=f^{-1}(\beta)$. Se $p:\left(Y_{\beta}, B_{\beta}\right) \longrightarrow\left(Y_{\alpha}, A_{\alpha}\right)$ é uma projeção, então $p^{\prime}=\left.p\right|_{\left(X_{\beta^{\prime}}, A_{\beta^{\prime}}\right)}:\left(X_{\beta^{\prime}}, A_{\beta^{\prime}}\right) \longrightarrow\left(X_{\alpha^{\prime}}, A_{\alpha^{\prime}}\right)$ é uma projeção e o seguinte diagrama comuta.

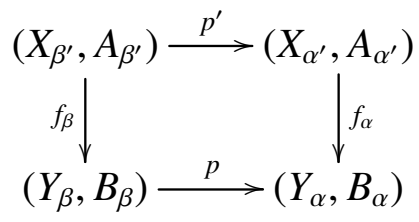

Lema 3.4.13. [13. Capítulo 9, Lema 2.11, p. 235]. Para qualquer $\alpha \in \operatorname{Cov}(X, A)$, a aplicação identidade $s_{\alpha} \mapsto s_{\alpha}$ é uma projeção. Se $p: s_{\beta} \mapsto s_{\alpha}$ e $p^{\prime}: s_{\gamma} \mapsto s_{\beta}$ são projeções, então $p \circ p^{\prime}: s_{\gamma} \mapsto s_{\alpha}$ é uma projeção.

Teorema 3.4.14. Dados $\alpha, \beta \in \operatorname{Cov}(X, A)$ tais que $\alpha \leq \beta$, então quaisquer duas projeções $p, p^{\prime}:\left(X_{\beta}, A_{\beta}\right) \longrightarrow\left(X_{\alpha}, A_{\alpha}\right)$ são contíguas. 
Demonstração: Sejam $s \in X_{\beta}$ e $x \in \bigcap_{v \in s} \beta_{v}$. Como $\beta_{v} \subset \alpha_{p(v)}$ e $\beta_{v} \subset \alpha_{p^{\prime}(v)}$, temos $x \in$ $\bigcap_{v \in s}\left(\alpha_{p(v)} \bigcap \alpha_{p^{\prime}(v)}\right)$. Então, $p(s)$ e $p^{\prime}(s)$ são faces de um mesmo simplexo em $X_{\alpha}$. De forma análoga, demonstra-se para o caso $s \in A_{\beta}$.

\subsection{A cohomologia de Čech}

A teoria de cohomologia de Čech é definida na categoria dos pares topológicos e suas aplicações. Dado um par topológico, construímos um par simplicial através do nervo de uma cobertura aberta e associamos um módulo de cohomologia simplicial. O módulo de cohomologia de Čech é definido com o auxílio do conceito de limite direto, no qual o conjunto de índices é o conjunto de todas as coberturas abertas do par dado.

W. S. Massey [27] mostra, na introdução de seu artigo How to give an exposition of the ČechAlexander-Spanier type homology theory, algumas das necessidades do uso da cohomologia de Čech, onde a teoria de cohomologia singular não é suficiente.

A cohomologia de Čech apresenta certas vantagens técnicas em relação à teoria singular. Nos limitaremos apenas à exposição da propriedade da continuidade apresentada adiante. Em espaços que são homotopicamente equivalentes a complexos CW, a cohomologia singular e de Čech coincidem, como comentado em [18].

\subsubsection{Os $R$-módulos de cohomologia de Čech}

Seja $(X, A)$ um par de espaços topológicos. Para cada $\alpha \in \operatorname{Cov}(X, A)$, denotemos por $H_{\alpha}^{q}(X, A)=H^{q}\left(X_{\alpha}, A_{\alpha} ; R\right)$ o $q$-ésimo $R$-módulo de cohomologia simplicial de $\left(X_{\alpha}, A_{\alpha}\right)$. E, para cada par $\alpha \leq \beta \in \operatorname{Cov}(X, A)$, seja $\pi_{\beta}^{\alpha}: H_{\alpha}^{q}(X, A) \longrightarrow H_{\beta}^{q}(X, A)$ o homomorfismo induzido por uma projeção $\left(X_{\beta}, A_{\beta}\right) \longrightarrow\left(X_{\alpha}, A_{\alpha}\right)$ (que independe da escolha, pelo Teorema 3.4.14).

Teorema 3.5.1. $\left\{H_{\alpha}^{q}(X, A), \pi_{\beta}^{\alpha}\right\}$ é um sistema direto sobre o conjunto dirigido $\operatorname{Cov}(X, A)$.

A demonstração segue imediatamente do Lema 3.4.13 e do Teorema 3.4.14.

Definição 3.5.2. O limite direto de $\left\{H_{\alpha}^{q}(X, A), \pi_{\beta}^{\alpha}\right\}$ é o q-ésimo $R$-módulo de cohomologia de Čech de $(X, A)$ e será denotado por $\check{H}^{q}(X, A ; R)$. 
Teorema 3.5.3. Sejam $f:(X, A) \longrightarrow(Y, B)$ aplicação de pares e $f^{-1}: \operatorname{Cov}(Y, B) \longrightarrow \operatorname{Cov}(X, A)$, dada por $\beta \mapsto f^{-1}(\beta)=\beta^{\prime}$. Para cada $\beta \in \operatorname{Cov}(Y, B)$, seja $f_{\beta}:\left(X_{\beta^{\prime}}, A_{\beta^{\prime}}\right) \longrightarrow\left(Y_{\beta}, B_{\beta}\right)$ a inclusão. Então, os homomorfismos induzidos $f_{\beta}^{*}: H^{q}\left(Y_{\beta}, B_{\beta} ; R\right) \longrightarrow H^{q}\left(X_{\beta^{\prime}}, A_{\beta^{\prime}} ; R\right)$ juntamente com $f^{-1}$ determinam uma aplicação $\Phi(f)^{q}$ entre os sistemas diretos $\left\{H_{\alpha}^{q}(Y, B), \pi_{\beta}^{\alpha}\right\}$ e $\left\{H_{\alpha^{\prime}}^{q}(X, A), \pi_{\beta^{\prime}}^{\alpha^{\prime}}\right\}$.

Demonstração: Segue da Proposição 3.4.12 que $f^{-1}$ é uma aplicação entre conjuntos dirigidos, isto é, preserva ordem. O diagrama 3.4 .1 da mesma proposição nos mostra que, se $\alpha \leq \beta$ então

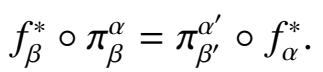

Definição 3.5.4. O limite direto de $\Phi(f)^{q}$ é denotado por $f^{*}: \check{H}^{q}(Y, B ; R) \longrightarrow \check{H}^{q}(X, A ; R) e$ chamado de homomorfismo induzido por $f$.

\subsubsection{Os axiomas de Eilenberg-Steenrod}

Denotemos por $\mathbf{T o p}^{2}$ a categoria dos pares topológicos junto com as aplicações contínuas de pares e por R-mod a categoria que tem como objetos os $R$-módulos e como morfismos os $R$-homomorfismos.

A demonstração dos resultados contidos no teorema a seguir pode ser encontrada em [10] e [13].

Teorema 3.5.5. Para todo $q \geq 0$, a função $\check{H}^{q}:$ Top $^{2} \rightarrow \boldsymbol{R}$-mod, que associa a cada par de espaços $(X, A)$, o R-módulo de cohomologia de $\check{C}$ ech $\breve{H}^{q}(X, A ; R)$ e a cada aplicação contínua de pares $f:(X, A) \rightarrow(Y, B)$ associa o homomorfismo induzido em cohomologia de Čech $f^{*}: \check{H}^{q}(Y, B ; R) \rightarrow \check{H}^{q}(X, A ; R)$, é um funtor contravariante, chamado q-funtor Cohomologia de Čch.

C.H. Dowker mostrou em [10], assim como S. Eilenberg e N. Steenrod em [13], que a Teoria de Cohomologia de Čech satisfaz os axiomas de Eilenberg-Steenrod. Em resumo, a Teoria de Cohomologia de Čech é a coleção dos $q$-funtores cohomologia de Čech $\left\{\check{H}^{q}\right\}$ que satisfaz os seguintes axiomas:

1. (Invariância Homotópica) Se $f_{1}, f_{2}:(X, A) \rightarrow(Y, B)$ são aplicações homotópicas, então $f_{1}^{*}=f_{2}^{*}$. 
2. (Exatidão) Dados os pares $(X, A)$ e $(A, B)$, existe um $R$-homomorfismo

$$
\delta: \check{H}^{q}(A, B ; R) \longrightarrow \check{H}^{q+1}(X, A ; R)
$$

que comuta com os homomorfismos induzidos em cohomologia de Čech e a sequência

$$
\cdots \stackrel{\delta}{\longrightarrow} \check{H}^{q+1}(X, A ; R) \stackrel{j^{*}}{\longrightarrow} \check{H}^{q+1}(X, B ; R) \stackrel{i^{*}}{\longrightarrow} \check{H}^{q+1}(A, B ; R) \stackrel{\delta}{\longrightarrow}
$$

é exata, na qual $i:(A, B) \rightarrow(X, B)$ e $j:(X, B) \rightarrow(X, A)$ são as inclusões.

3. (Excisão) Se $(X, A)$ é um par de espaços e $U$ é um subconjunto de $X$ tal que $\bar{U} \subset \operatorname{Int}(A)$, então, para todo $q \geq 0$, o homomorfismo

$$
i^{*}: \check{H}^{q}(X, A ; R) \rightarrow \check{H}^{q}(X-U, A-U ; R)
$$

induzido da inclusão é um isomorfismo.

4. (Dimensão) Se $X=\left\{x_{0}\right\}$ consiste de apenas um ponto, então

$$
\check{H}^{q}\left(\left\{x_{0}\right\} ; R\right)=\left\{\begin{array}{cc}
R, & q=0 ; \\
0, & q>0 .
\end{array}\right.
$$

A teoria de cohomologia de Čech, além de satisfazer os axiomas de Eilenberg-Steenrod, possui uma propriedade muito especial, conhecida como Axioma da Continuidade, que pode ser descrita como segue: se o par compacto $(X, A)^{2}$ for o limite inverso $5^{3}$ de uma família de pares compactos $\left(X_{\alpha}, A_{\alpha}\right)$, então o $R$-módulo de cohomologia de Čech de $(X, A)$ é o limite direto dos $R$-módulos de cohomologia de Čech dos pares $\left(X_{\alpha}, A_{\alpha}\right)$. Na categoria dos pares compactos, a teoria de cohomologia de Čech é a única que possui esta propriedade, isto é, qualquer outra teoria de cohomologia onde se verifica o axioma da continuidade na categoria dos pares compactos é equivalente à teoria da cohomologia de Čech. Mais precisamente, temos os seguintes teoremas.

\footnotetext{
${ }^{2}(X, A)$ é um par compacto se $X$ é um espaço topológico de Hausdorff compacto e $A \subset X$ é fechado.

${ }^{3}$ Vide Capítulo 6, Seção 6.3
} 
Teorema 3.5.6. [13. Capítulo 10, Teorema 3.1, p. 261]. Seja $\left\{\left(X_{m}, A_{m}\right), \pi\right\}$ um sistema inversd de pares topológicos compactos indexado em um conjunto dirigido $M$. Sejam $(X, A)$ o limite inverso do sistema acima (isto é, $X$ é o limite inverso de $\left\{X_{m}, \pi\right\}$ e $A$ é o limite inverso de $\left\{A_{m}, \pi\right\}$ ) e $\pi_{m}:(X, A) \longrightarrow\left(X_{m}, A_{m}\right)$ as projeções $\bigsqcup^{5}$ Então, $\left\{\check{H}^{p}\left(X_{m}, A_{m}\right), \pi_{m}^{n}=\pi_{n}^{m *}\right\}$ é um sistema direto $e$ $\left\{\pi_{m}^{*}\right\}$ induz um isomorfismo

$$
\underset{\lim }{\longrightarrow}\left\{\check{H}^{p}\left(X_{m}, A_{m}\right)\right\} \stackrel{\cong}{\longrightarrow} \check{H}^{p}\left(\lim _{\longleftarrow}\left\{\left(X_{m}, A_{m}\right)\right\}\right),
$$

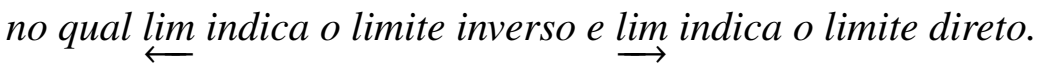

Uma consequência do teorema acima (Axioma da Continuidade) é o seguinte:

Teorema 3.5.7. [23. Teorema da Redução]. Seja $(X, A)$ um par compacto Hausdorffe $F \subset X$ um subespaço fechado. Se $u \in \check{H}^{*}(X, A)$ é levado pela induzida da inclusão em $\left.u\right|_{(F, F \cap A)}=0 \in$ $\check{H}^{*}(F, F \cap A)$, então existe um aberto $\mathcal{U} \subset X$ tal que $\mathcal{U}$ contém $F$ e $\left.u\right|_{(\overline{\mathcal{U}}, \overline{\mathcal{U}} \cap A)}=0$.

Como comentado em [4], o resultado acima é válido para $X$ e $A$ paracompactos.

Teorema 3.5.8. [13] Capítulo X, Teorema 12.2, p. 288]. Qualquer teoria de cohomologia definida na categoria dos pares compactos que satisfaz o axioma da continuidade (Teorema 3.5.6 é isomorfa à teoria de cohomologia de Čech.

Existe um isomorfismo natural entre os $R$-módulos de cohomologia de Čech e os $R$-módulos de cohomologia de Alexander ${ }^{6}$. No artigo Cohomology theory for General Spaces de E. H. Spanier [34], vemos que as cohomologias de Alexander e de Čech coincidem para espaços compactos, através da unicidade dada no Teorema 3.5.8. No artigo Continuous Connectivity groups in terms of limit group de W. Hurewicz, J. Dugundji e C. H. Dowker [20], mostra-se que as cohomologias de Alexander e de Čech coincidem em espaços paracompactos. Finalmente, no artigo Homology Group of Relations de C. H. Dowker [11], os módulos de Čech e de Alexander de um par topológico são isomorfos quando construídos sobre o mesmo conjunto de coberturas. Uma definição equivalente é dada a seguir (Vide [11, p. 90] e [33, Capítulo 6, pg. 311]).

\footnotetext{
${ }^{4}$ Vide Capítulo 6. Seção 6.2

${ }^{5}$ Vide Capítulo 6 Seção $\overline{\overline{6.3}}$

${ }^{6}$ Vide Capítulo 6 , Seção 6.4
} 
Definição 3.5.9. Seja $\alpha:\left(V_{\alpha}, V_{\alpha}^{A}\right) \longrightarrow \mathcal{P}(X)$ uma cobertura aberta para o par topológico $(X, A)$, no qual $\mathcal{P}(X)$ denota o conjunto das partes de $X$. Um par Vietonis $L=\left(L_{\alpha}, K_{\alpha}\right)$ de $(X, A)$ com relação à cobertura $\alpha$ é um par simplicial definido como segue: o conjunto de vértices é os pontos de $X$, um simplexo de $L_{\alpha}$ é uma coleção finita de pontos de X contida em algum aberto $\alpha_{v}$, para $v \in V_{\alpha}$, e um simplexo de $K_{\alpha}$ é uma coleção finita de pontos de A contida em algum aberto $\alpha_{w}$, para algum $w \in V_{\alpha}^{A}$.

Dadas duas coberturas $\alpha \leq \beta$ do par $(X, A)$, uma aplicação $\pi_{\beta}^{\alpha}:\left(L_{\beta}, K_{\beta}\right) \longrightarrow\left(L_{\alpha}, K_{\alpha}\right) e ́$ chamada uma projeção quando:

(i) $\pi_{\beta}^{\alpha}$ restrita aos 0 -simplexos (pontos de X) é a identidade.

(ii) Um simplexo de $L_{\beta}$ é levado em um simplexo de $L_{\alpha}$.

(iii) Um simplexo de $K_{\beta}$ é levado em um simplexo de $K_{\alpha}$.

Podemos ter mais de uma projeção entre $\left(L_{\beta}, K_{\beta}\right)$ e $\left(L_{\alpha}, K_{\alpha}\right)$, porém estas são contíguas. Os módulos $H^{q}\left(L_{\alpha}, K_{\alpha}\right)$ juntamente com as projeções $\pi_{\beta}^{\alpha *}$ formam um sistema direto cujo limite direto é $\bar{H}^{q}(X, A)$, o q-ésimo módulo de cohomologia de Alexander.

OBS 3.5.10. A equivalência da Definição 3.5.9 com a definição dada no Capítulo 6 Seção 6.4 é comentada em Dowker [11] e provada por Spanier [33, Capítulo 6, Corolário 2, p. 312].

Através do isomorfismo entre a cohomologia de Alexander e a cohomologia de Čech e, usando a sequência de Mayer-Vietoris dada no Capítulo 6. Seção 6.4, obtemos uma sequência de Mayer-Vietoris para a cohomologia de Čech.

Proposição 3.5.11 (Mayer-Vietoris). Sejam $X_{1}, X_{2}$ abertos em $X_{1} \cup X_{2}$, então sequência

$$
\cdots \longrightarrow \check{H}^{q}\left(X_{1} \cup X_{2}\right) \stackrel{\left(i_{1}^{*}, i_{2}^{*}\right)}{\longrightarrow} \check{H}^{q}\left(X_{1}\right) \oplus \check{H}^{q}\left(X_{2}\right) \stackrel{\left(j_{1}^{*}-j_{2}^{*}\right)}{\longrightarrow} \check{H}^{q}\left(X_{1} \cap X_{2}\right) \stackrel{\delta}{\longrightarrow} \check{H}^{q+1}\left(X_{1} \cup X_{2}\right) \longrightarrow \cdots
$$

é exata, na qual $\delta$ é um homomorfismo conectante e $i_{1}: X_{1} \longrightarrow X_{1} \cup X_{2}, i_{2}: X_{2} \longrightarrow X_{1} \cup X_{2}$, $j_{1}: X_{1} \cap X_{2} \longrightarrow X_{1}$ e $j_{2}: X_{1} \cap X_{2} \longrightarrow X_{2}$ são inclusões.

OBS 3.5.12. Dold estabelece uma sequência de Mayer-Vietoris para a cohomologia de Čech no caso em que a tríada $\left(X, X_{1}, X_{2}\right)$ é tal que $X$ é um ENR e $X_{1}, X_{2}$ são subespaços localmente compactos com propriedades especiais (Vide [8] Capítulo VIII, Proposição 6.13, p. 285]). 


\subsubsection{O produto cup $\cup$}

Apresentamos aqui o produto cup para a cohomologia de Čech. Uma prova direta será dada para o caso de subespaços fechados. Sejam $(X, A),(X, B)$ e $(X, A \cup B)=(X, C)$ pares de espaços topológicos, nos quais $A$ e $B$ são fechados.

Lema 3.5.13. $\operatorname{Cov}^{\prime}(X, A)=\left\{\alpha \in \operatorname{Cov}(X, A) ; \alpha_{v} \cap A \neq \emptyset\right.$, para todo $\left.v \in V_{\alpha}^{A}\right\}$ é cofinal em $\operatorname{Cov}(X, A)$.

Demonstração: Sejam $\alpha \in \operatorname{Cov}(X, A)$ e $V^{\prime}$ o conjunto de todos os índices $v \in V_{\alpha}^{A}$ tal que $\alpha_{v} \cap A \neq \emptyset$. Considere $\beta$ a cobertura indexada por $\left(V_{\alpha}, V^{\prime}\right)$ e definida por $\beta_{v}=\alpha_{v}-A$, se $v \in V_{\alpha}-V^{\prime}$ e por $\beta_{v}=\alpha_{v}$, se $v \in V^{\prime}$. Então $\beta \in \operatorname{Cov}^{\prime}(X, A)$ e $\alpha \leq \beta$.

Seja $\gamma \in \operatorname{Cov}^{\prime}(X, C)$. Então, $\alpha=\left(V_{\gamma}, V_{\gamma}^{\prime A}\right) \in \operatorname{Cov}^{\prime}(X, A)$ e $\beta=\left(V_{\gamma}, V_{\gamma}^{\prime B}\right) \in \operatorname{Cov}^{\prime}(X, B)$. Se $s \in C_{\gamma}$, então $\left(\bigcap \gamma_{v}\right) \cap C \neq \emptyset$. Logo, $s \in A_{\alpha}$ ou $s \in B_{\beta}$ e, então, $C_{\gamma}=A_{\alpha} \cup B_{\beta}$. Dada uma projeção $p_{C}:\left(X_{\gamma^{\prime}}, C_{\gamma^{\prime}}\right) \longrightarrow\left(X_{\gamma}, C_{\gamma}\right)$, temos as projeções

$$
p_{A}=\left.p_{C}\right|_{\left(X_{\alpha^{\prime}}, A_{\alpha^{\prime}}\right)}:\left(X_{\alpha^{\prime}}, A_{\alpha^{\prime}}\right) \longrightarrow\left(X_{\alpha}, A_{\alpha}\right) \text { e } p_{B}=\left.p_{C}\right|_{\left(X_{\beta^{\prime}}, B_{\beta^{\prime}}\right)}:\left(X_{\beta^{\prime}}, B_{\beta^{\prime}}\right) \longrightarrow\left(X_{\beta}, B_{\beta}\right),
$$

no qual $C_{\gamma^{\prime}}=A_{\alpha^{\prime}} \cup B_{\beta^{\prime}}$ e $C_{\gamma}=A_{\alpha} \cup B_{\beta}$. Note que o diagrama

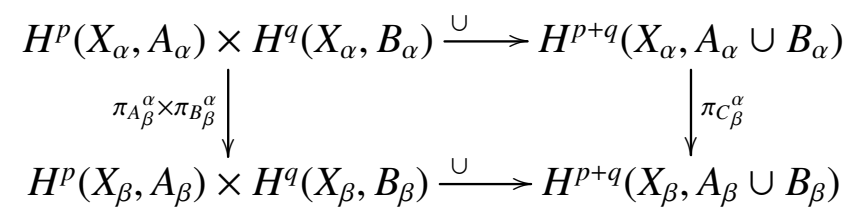

é comutativo, no qual $\pi_{C}^{\alpha}$ é o homomorfismo induzido de alguma projeção $p_{C}$ e $\pi_{A_{\beta}}^{\alpha}, \pi_{B \beta}^{\alpha}$ são os homomorfismos induzidos das restrições da projeção $p_{C}$.

Lema 3.5.14. $\left\{H^{p}\left(X_{\alpha}, A_{\alpha}\right) \times H^{q}\left(X_{\alpha}, B_{\alpha}\right), \pi_{A_{\beta}}^{\alpha} \times \pi_{B_{\beta}}^{\alpha}\right\}$ é um sistema direto sobre $\operatorname{Cov}(X)$. Mais ainda, o limite direto do sistema acima é $\check{H}^{p}(X, A) \times \check{H}^{q}(X, B)$.

Demonstração: Para cada $\alpha \in \operatorname{Cov}(X)$ associamos um $R$-módulo $H^{p}\left(X_{\alpha}, A_{\alpha}\right) \times H^{q}\left(X_{\alpha}, B_{\alpha}\right)$. E, para cada par $\alpha, \beta \in \operatorname{Cov}(X)$ tal que $\alpha \leq \beta$, associamos um $R$-homomorfismo $\pi_{A_{\beta}}^{\alpha} \times \pi_{B_{\beta}}^{\alpha}$ tal que:

- $\pi_{A_{\alpha}}^{\alpha} \times \pi_{B_{\alpha}}^{\alpha}=I d \times I d=I d$. 
- Se $\alpha \leq \beta \leq \gamma$ então $\left(\pi_{A \gamma}^{\beta} \times \pi_{B \gamma}^{\beta}\right) \circ\left(\pi_{A_{\beta}^{\alpha}}^{\alpha} \times \pi_{B \beta}^{\alpha}\right)=\left(\pi_{A \gamma}^{\beta} \circ \pi_{A_{\beta}^{\alpha}}^{\alpha}\right) \times\left(\pi_{B \gamma}^{\beta} \circ \pi_{B \beta}^{\alpha}\right)=\pi_{A \gamma}^{\alpha} \times \pi_{B \gamma}^{\alpha}$.

Denotemos por $H^{p, q}$ o $R$-módulo $\frac{\bigoplus_{\alpha \in \operatorname{Cov}(X)} H^{p}\left(X_{\alpha}, A_{\alpha}\right) \times H^{q}\left(X_{\alpha}, B_{\alpha}\right)}{Q^{p, q}}$, no qual $Q^{p, q}$ é o $R$ módulo gerado pelas relações $\pi_{A \beta}^{\alpha} \times \pi_{B \beta}^{\alpha}\left(g^{\alpha}, h^{\alpha}\right)-\left(g^{\alpha}, h^{\alpha}\right)$, para todo $\alpha \in \operatorname{Cov}(X)$.

Consideremos a aplicação $\varphi: H^{p, q} \longrightarrow \check{H}^{p}(X, A) \times \check{H}^{q}(X, B)$ definida por

$$
\sum_{\alpha \in \operatorname{Cov}(X)}\left(g^{\alpha}, h^{\alpha}\right)+Q^{p, q} \mapsto \sum_{\alpha \in \operatorname{Cov}(X)} g^{\alpha}+Q^{p} \times \sum_{\alpha \in \operatorname{Cov}(X)} h^{\alpha}+Q^{q}
$$

Claramente, $\varphi$ é sobrejetora e leva relações em relações. Logo, $\varphi$ é um isomorfismo. Portanto, o limite direto do sistema direto $\left\{H^{p}\left(X_{\alpha}, A_{\alpha}\right) \times H^{q}\left(X_{\alpha}, B_{\alpha}\right), \pi_{A_{\beta}}^{\alpha} \times \pi_{B \beta}^{\alpha}\right\}$ é $\check{H}^{p}(X, A) \times \check{H}^{q}(X, B)$.

Lema 3.5.15. $\Phi(\cup):\left\{H^{p}\left(X_{\alpha}, A_{\alpha}\right) \times H^{q}\left(X_{\alpha}, B_{\alpha}\right), \pi_{A_{\beta}}^{\alpha} \times \pi_{B \beta}^{\alpha}\right\} \longrightarrow\left\{H^{p+q}\left(X_{\alpha}, C_{\alpha}=A_{\alpha} \cup B_{\alpha}\right), \pi_{C_{\beta}^{\alpha}}^{\alpha}\right\}$, formada por $\rho: \operatorname{Cov}(X) \longrightarrow \operatorname{Cov}^{\prime}(X, C)$ tal que $\rho(\alpha)=\alpha^{\prime}=\left(V_{\alpha}, V_{\alpha}^{\prime A} \cup V_{\alpha}^{\prime B}\right)$ e pelos produtos $\cup$, é uma aplicação entre sistemas diretos.

Demonstração: A aplicação $\rho$ é uma aplicação entre conjuntos dirigidos. De fato, se $\alpha \leq \beta \in$ $\operatorname{Cov}(X)$, para todo $v \in V_{\beta}$, existe $w \in V_{\alpha}$ tal que $\beta_{v} \subset \alpha_{w}$. Analogamente, se $v \in V_{\beta}^{\prime A} \cup V_{\beta}^{\prime B}$, então existirá $w \in V_{\alpha}^{\prime A} \cup V_{\alpha}^{\prime B}$ tal que $\beta_{v} \subset \alpha_{w}$. Logo, $\rho(\alpha)=\alpha^{\prime} \leq \beta^{\prime}=\rho(\beta)$. Como o diagrama 3.5.1 é comutativo, segue que $\Phi(\cup)$ é uma aplicação entre sistemas diretos.

Definição 3.5.16. O limite direto de $\Phi(\cup)$ é o produto cup (para fechados) para a cohomologia de $\check{C} e c h$,

$$
\cup: \check{H}^{p}(X, A) \times \check{H}^{q}(X, B) \longrightarrow \check{H}^{p+q}(X, A \cup B)
$$

OBS 3.5.17. Para o caso em que os subespaços A e B são subconjuntos abertos de X, utilizando o isomorfismo entre a cohomologia de Alexander e a cohomologia de Čech e o produto cup dado no Capítulo 6. Seção 6.4. obtemos o produto cup (para abertos) para a cohomologia de Čech,

$$
\cup: \check{H}^{p}(X, A) \times \check{H}^{q}(X, B) \longrightarrow \check{H}^{p+q}(X, A \cup B)
$$




\section{Capítulo}

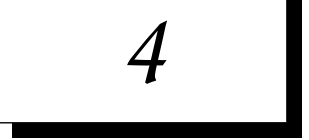

\section{Classes Características}

Esse capítulo dedica-se à exposição das classes características de Stiefel-Whitney de um fibrado vetorial e da classe característica de Euler de um par fibrado. Para isso, resultados importantes como a fórmula de Künneth, o teorema de Leray-Hirsch e o isomorfismo de Thom, são utilizados. As referências básicas para este capítulo são Characteristic Classes [28] de J.W. Milnor e J.D.Stasheff, Algebraic Topology [18] e Vector Bundles and K-Theory [17] de A. Hatcher, Algebraic Topology [33] de E. H. Spanier, Foundations of Algebraic Topology [13] de S. Eilenberg e N. Steenrod e Fibre Bundles [19] de D. Husemoller.

Todos os módulos de cohomologia usados neste capítulo possuem coeficientes em $\mathbb{Z}_{2}$, a menos que se diga o contrário.

\subsection{Fórmulas de Künneth}

Para o desenvolvimento desta seção será necessário apresentarmos uma nova operação entre classes de cohomologia, o produto cross.

Sejam $(X, A)$ e $(Y, B)$ pares topológicos, no qual $A \subset X$ e $B \subset Y$ são conjuntos abertos. Consideremos as projeções $p_{1}:(X \times Y, A \times Y) \longrightarrow(X, A), p_{2}:(X \times Y, X \times B) \longrightarrow(Y, B)$, no qual $p_{1}(x, y)=x$ e $p_{2}(x, y)=y$. O produto cross entre $a \in \check{H}^{p}(X, A)$ e $b \in \check{H}^{q}(Y, B)$ é a classe $a \times b \in \check{H}^{p+q}(X \times Y,(A \times Y) \cup(X \times B))$, definida por, $a \times b=p_{1}^{*}(a) \cup p_{2}^{*}(b)$. Usaremos a notação $(X, A) \times(Y, B)$ para expressar o $\operatorname{par}(X \times Y,(A \times Y) \cup(X \times B))$. 
Consideremos agora a sequência exata de cohomologia da tripla $\left(\mathbb{R}, \mathbb{R}_{0}, \mathbb{R}_{-}\right)$, no qual $\mathbb{R}_{0}=$ $\mathbb{R}-\{0\}$. (Vide [13, Capítulo I, Teorema 10.2 C, p. 29]):

$$
\cdots \longrightarrow \check{H}^{q-1}\left(\mathbb{R}_{0}, \mathbb{R}_{-}\right) \stackrel{\delta}{\longrightarrow} \check{H}^{q}\left(\mathbb{R}, \mathbb{R}_{0}\right) \stackrel{j^{*}}{\longrightarrow} \check{H}^{q}\left(\mathbb{R}, \mathbb{R}_{-}\right) \stackrel{i^{*}}{\longrightarrow} \check{H}^{q}\left(\mathbb{R}_{0}, \mathbb{R}_{-}\right) \longrightarrow \cdots
$$

Pelo axioma da excisão, tomando $U=\mathbb{R}_{-}$, obtemos $\check{H}^{0}\left(\mathbb{R}_{0}, \mathbb{R}_{-}\right) \cong \check{H}^{0}\left(\mathbb{R}_{+}\right)$. Seja $e \in \check{H}^{1}\left(\mathbb{R}, \mathbb{R}_{0}\right)$ a imagem de $1 \in \check{H}^{0}\left(\mathbb{R}_{+}\right) \cong \mathbb{Z}_{2}$ pela composição $\check{H}^{0}\left(\mathbb{R}_{+}\right) \cong \check{H}^{0}\left(\mathbb{R}_{0}, \mathbb{R}_{-}\right) \stackrel{\delta}{\longrightarrow} \check{H}^{1}\left(\mathbb{R}, \mathbb{R}_{0}\right)$. Defina $e^{n}=\underbrace{e \times \cdots \times e}_{n \text { vezes }} \in \check{H}^{n}\left(\mathbb{R}^{n}, \mathbb{R}_{0}^{n}\right)\left(\right.$ Note que $\left.\mathbb{R} \times \mathbb{R}_{0} \cup \mathbb{R}_{0} \times \mathbb{R}=\mathbb{R}_{0}^{2}\right)$.

Teorema 4.1.1. Para qualquer par topológico $(X, A)$ com $A \subset X$ aberto, temos o isomorfismo $\check{H}^{m}(X, A) \longrightarrow \check{H}^{m+n}\left((X, A) \times\left(\mathbb{R}^{n}, \mathbb{R}_{0}^{n}\right)\right)$, definido por $a \mapsto a \times e^{n}$.

Demonstração: (i) Caso $n=1$. Suponhamos que $A=\emptyset$ e fixemos um elemento $a \in \check{H}^{m}(X)$. Queremos mostrar que $\check{H}^{m}(X) \longrightarrow \check{H}^{m+1}\left(X \times \mathbb{R}, X \times \mathbb{R}_{0}\right)$, dado por $y \mapsto y \times e$ é isomorfismo. $\mathrm{O}$ diagrama abaixo é comutativo, os isomorfismos são dados por excisão e $\check{H}^{m}\left(X \times \mathbb{R}_{+}\right) \cong \check{H}^{m}(X)$, pois $\mathbb{R}_{+}$é contrátil.

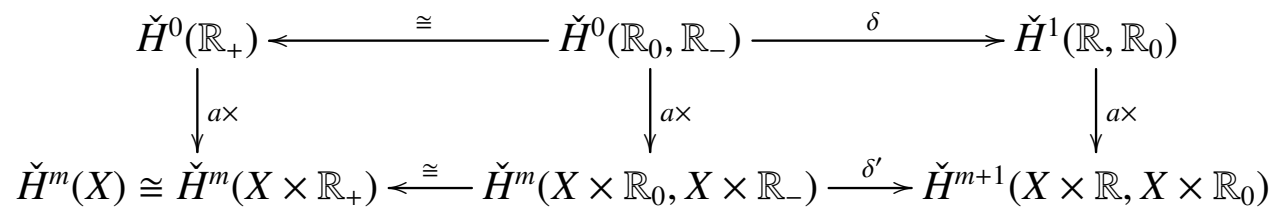

Note que $\delta^{\prime}$ é um isomorfismo, pois $\mathbb{R}$ e $\mathbb{R}$ _ são contráteis. De fato:

$\longrightarrow \underbrace{\check{H}^{m-1}\left(X \times \mathbb{R}, X \times \mathbb{R}_{-}\right)}_{\cong 0} \longrightarrow \check{H}^{m}\left(X \times \mathbb{R}_{0}, X \times \mathbb{R}_{-}\right) \stackrel{\delta^{\prime}}{\longrightarrow} \check{H}^{m+1}\left(X \times \mathbb{R}, X \times \mathbb{R}_{0}\right) \longrightarrow \underbrace{\check{H}^{m+1}\left(X \times \mathbb{R}, X \times \mathbb{R}_{-}\right)}_{\cong 0} \longrightarrow$.

Então,

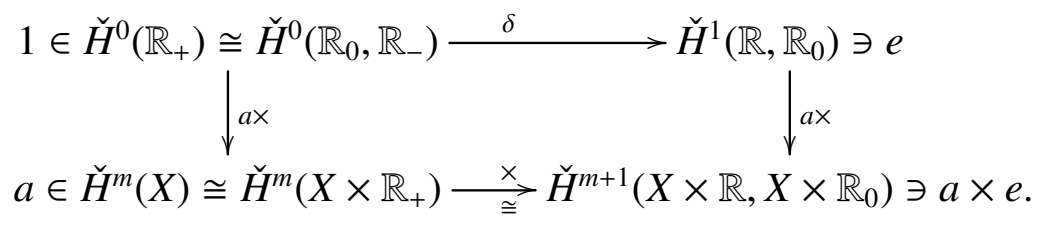

Agora, suponhamos que $A$ seja não vazio. Tem-se,

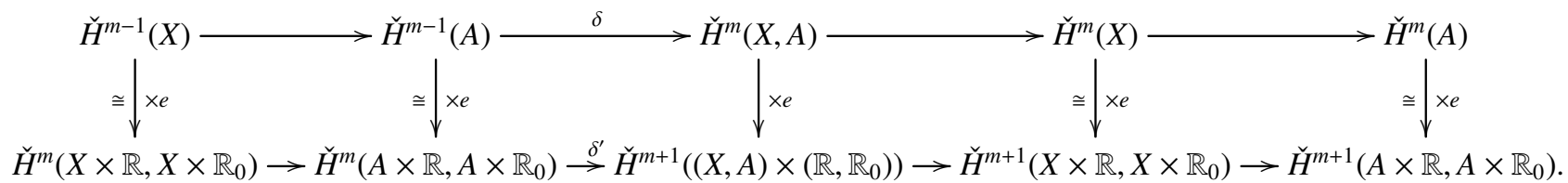


O resultado segue pelo Lema dos Cinco.

(ii) Caso $n>1$. A prova será feita por indução. Suponhamos válido o resultado para todo natural menor do que $n$. Então, $\check{H}^{m}(X, A) \longrightarrow \check{H}^{m+n}\left((X, A) \times\left(\mathbb{R}^{n}, \mathbb{R}_{0}^{n}\right)\right)$, dada por $a \mapsto a \times e^{n}$, pode ser escrito como sendo a composição das seguintes aplicações:

$$
\begin{aligned}
\check{H}^{m}(X, A) & \longrightarrow \check{H}^{m+n-1}\left((X, A) \times\left(\mathbb{R}^{n-1}, \mathbb{R}_{0}^{n-1}\right)\right), & a \mapsto a \times e^{n-1}, \\
\check{H}^{m+n-1}\left((X, A) \times\left(\mathbb{R}^{n-1}, \mathbb{R}_{0}^{n-1}\right)\right) & \longrightarrow \check{H}^{m+n}\left((X, A) \times\left(\mathbb{R}^{n}, \mathbb{R}_{0}^{n}\right)\right), & y \mapsto y \times e^{n},
\end{aligned}
$$

as quais são isomorfismos.

Uma versão da fórmula de Künneth para a cohomologia de Čech é dada a seguir.

Teorema 4.1.2. [28, Apêndice A, Teorema A.6, p. 268]. Seja Y um complexo CW finito e $X$ um espaço topológico de Hausdorff. Então,

$$
\bigoplus_{r+s=n} \check{H}^{r}\left(X ; \mathbb{Z}_{2}\right) \otimes \check{H}^{s}\left(Y ; \mathbb{Z}_{2}\right) \stackrel{\times}{\longrightarrow} \check{H}^{n}\left(X \times Y ; \mathbb{Z}_{2}\right)
$$

é um isomorfismo, no qual $\otimes$ é o produto tensoria ${ }^{2}$

Demonstração: A prova será feita por indução na dimensão e no número de células de $Y$. Se $Y$ for um ponto, a demonstração é imediata. Suponhamos, como hipótese de indução que o teorema seja válido para complexos CW com dimensão menor e com menos células do que $Y$.

Seja $D$ a célula aberta de maior dimensão de $Y$ e seja $Y_{1}=Y-D$. Por hipótese de indução,

$$
\times^{\prime}: \bigoplus_{r+s=n} \check{H}^{r}\left(X ; \mathbb{Z}_{2}\right) \otimes \check{H}^{s}\left(Y_{1} ; \mathbb{Z}_{2}\right) \stackrel{\times}{\longrightarrow} \check{H}^{n}\left(X \times Y_{1} ; \mathbb{Z}_{2}\right)
$$

é um isomorfismo. Da sequência exata longa do par $\left(Y, Y_{1}\right)$, temos a seguinte sequência exata:

$$
\check{H}^{s-1}\left(Y_{1}\right) \longrightarrow \check{H}^{s}\left(Y, Y_{1}\right) \longrightarrow \check{H}^{s}(Y) \longrightarrow \check{H}^{s}\left(Y_{1}\right) \longrightarrow \check{H}^{s+1}\left(Y, Y_{1}\right)
$$

Aplicando o funtor $\check{H}^{r}(X) \otimes 3^{3}$ na sequência acima e somando termo a termo de forma que

\footnotetext{
${ }^{1}$ Vide Capítulo 6 Seção 6.5

${ }^{2}$ Vide Definição 6.1

${ }^{3}$ Vide capítulo 6, Seção 6.1
} 
$r+s=n$, obtemos a seguinte sequência exata:

$\bigoplus_{r+s=n} \check{H}^{r}(X) \otimes \check{H}^{s-1}\left(Y_{1}\right) \longrightarrow \bigoplus_{r+s=n} \check{H}^{r}(X) \otimes \check{H}^{s}\left(Y, Y_{1}\right) \longrightarrow \bigoplus_{r+s=n} \check{H}^{r}(X) \otimes \check{H}^{s}(Y) \longrightarrow \bigoplus_{r+s=n} \check{H}^{r}(X) \otimes \check{H}^{s}\left(Y_{1}\right) \longrightarrow \bigoplus_{r+s=n} \check{H}^{r}(X) \otimes \check{H}^{s+1}\left(Y, Y_{1}\right)$

Então, temos o seguinte diagrama

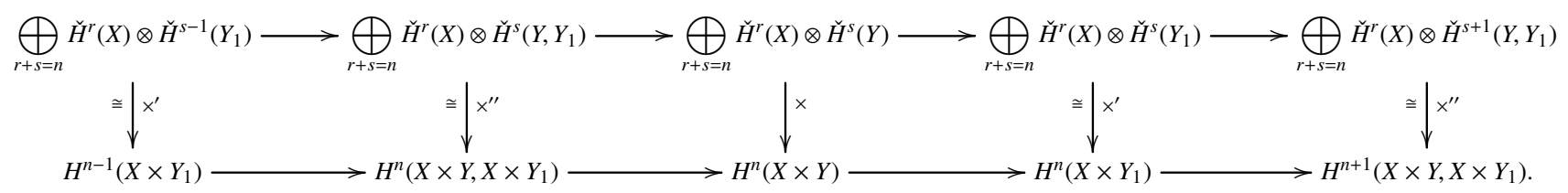

no qual as aplicações $\times^{\prime}$ são isomorfismos, por hipótese de indução. Note que,

$$
\check{H}^{*}\left(Y, Y_{1}\right) \cong \check{H}^{*}(Y, Y-\{p t o\}) \cong \check{H}^{*}(D, D-\{p t o\}) \cong \check{H}^{*}\left(\mathbb{R}^{n}, \mathbb{R}_{0}^{n}\right)
$$

Da mesma forma, $\check{H}^{*}\left(X \times Y, X \times Y_{1}\right) \cong \check{H}^{*}\left(X \times \mathbb{R}^{n}, X \times \mathbb{R}_{0}^{n}\right)$. Então, segue do Teorema 4.1.1 que as aplicações $\times^{\prime \prime}$ são isomorfismos. Logo, pelo Lema dos Cinco, $\times$ é um isomorfismo.

Uma outra versão da fórmula de Künneth para pares compactos é apresentada abaixo.

Teorema 4.1.3. [33. Capítulo 6, E5, pg. 360]. Sejam $(X, A)$ e $(Y, B)$ dois pares compactos. Então, $\bigoplus_{r+s=n} \check{H}^{r}\left(X, A ; \mathbb{Z}_{2}\right) \otimes \check{H}^{s}\left(Y, B ; \mathbb{Z}_{2}\right) \stackrel{\times}{\longrightarrow} \check{H}^{n}\left((X, A) \times(Y, B) ; \mathbb{Z}_{2}\right)$ é um isomorfismo.

\subsection{O teorema de Leray-Hirsch}

O teorema de Leray-Hirsch é a principal ferramenta para se definir as classes características de Stiefel-Whitney. Em sua demonstração, vemos a importância da fórmula de Künneth.

Teorema 4.2.1 (Leray-Hirsch). , [19] Capítulo 16, Teorema 1.1, p. 231]. Seja $(E, \pi, B, F)$ um fibrado localmente trivial e finito (isto é, possui um número finito de cartas), no qual $F$ é um complexo $C W$ finito. Suponhamos que $\check{H}^{*}(F)$ seja um $\mathbb{Z}_{2}$-módulo livre finitamente gerado. Sejam $\left(a_{1}, \ldots, a_{r}\right) \in \check{H}^{n(1)}(E) \times \cdots \times \check{H}^{n(r)}(E)$ elementos tais que $j_{b}^{*}\left(a_{1}\right), \ldots, j_{b}^{*}\left(a_{r}\right)$ formam uma $\mathbb{Z}_{2}$-base para $\check{H}^{*}\left(F_{b}\right)$, no qual $j_{b}: F_{b}=\pi^{-1}(b) \longrightarrow$ Eé a inclusão, para todo $b \in$ B. Então, $\check{H}^{*}(E)$ é um $\check{H}^{*}(B)$-módulo gerado por $a_{1}, \ldots, a_{r}$, no qual $x y=x \cup \pi^{*}(y)$, para $x \in \check{H}^{*}(E) e$ $y \in \check{H}^{*}(B)$. 
Demonstração: Seja $U$ um aberto de $B$ tal que $U \times F \cong \pi^{-1}(U)$. Assim, pela fórmula de Künneth, $\bigoplus_{i+j=q} \check{H}^{i}(U) \otimes \check{H}^{j}(F) \stackrel{\cong}{\longrightarrow} \check{H}^{q}\left(\pi^{-1}(U)\right),\left.a \otimes c \mapsto \pi\right|_{U} ^{*}(a) \cup j_{U}^{*}(c)$, no qual $j_{U}: \pi^{-1}(U) \hookrightarrow E$. Então, $\check{H}^{*}\left(\pi^{-1}(U)\right)$ é um $\check{H}^{*}(U)$-módulo finitamente gerado por $j_{U}^{*}\left(a_{1}\right), \ldots, j_{U}^{*}\left(a_{r}\right)$ e o teorema é válido para um tal aberto $U$.

Sejam $\{U, h\}$ e $\left\{V, h^{\prime}\right\}$ duas cartas locais. É suficiente provar que se o resultado é válido para $U, V$ e $U \cap V$, então será válido para $U \cup V$. Usaremos a sequência de Mayer- Vietoris para provar este caso. Considere $K^{q}(U)=\sum_{1 \leq i \leq r} \check{H}^{q-n(i)}(U) x_{i}$ e $L^{q}(U)=\check{H}^{q}\left(\pi^{-1}(U)\right)$, nos quais $x_{i}$ são indeterminadas de grau $n(i)$. Note que, o teorema vale para um aberto $W$ se, e somente se, a função $\theta_{W}: K^{q}(W) \longrightarrow L^{q}(W),\left.\sum_{i} c_{i} x_{i} \mapsto \sum_{i} \pi\right|_{W} ^{*}\left(c_{i}\right) \cup j_{W}^{*}\left(a_{i}\right)$, for isomorfismo. Considere o seguinte diagrama:

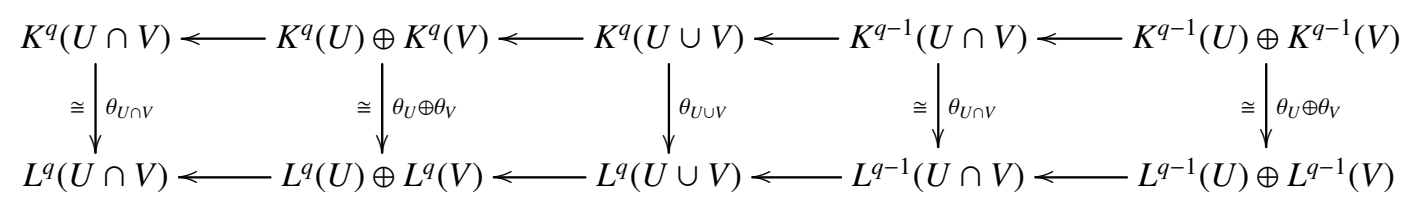

Desde que o teorema é válido para $U, V$ e $U \cap V$, temos os isomorfismos $\theta_{U \cap V}$ e $\theta_{U} \oplus \theta_{V}$. Pelo Lema dos Cinco, $\theta_{U \cup V}$ é um isomorfismo. O resultado segue por indução.

OBS 4.2.2. O teorema vale para fibrados localmente triviais não finitos com fibra $F$ paracompacto, como comentado em [19] Capítulo 16, Observação 1.2, p. 232].

Definição 4.2.3. Um par fibrado consiste de um fibrado localmente trivial $\xi=(E, \pi, B, F)$ com fibra $F$, junto com um subespaço $E^{\prime} \subset E$ tal que $\pi^{\prime}: E^{\prime} \longrightarrow B$ é um fibrado com fibra $F^{\prime} \subset F$, com trivializações locais para $E^{\prime}$ obtidas por restrições das trivializações locais para E. Usaremos a seguinte notação para o par fibrado: $\left(F, F^{\prime}\right) \hookrightarrow\left(E, E^{\prime}\right) \longrightarrow B$.

Exemplo 4.2.4. Sejam $\pi: E \longrightarrow B$ um fibrado com fibra $D^{n}$ e $E^{\prime}$ a reunião dos bordos das fibras. Então, $\left(D^{n}, S^{n-1}\right) \hookrightarrow\left(E, E^{\prime}\right) \longrightarrow B$ é chamado parfibrado em disco.

Definição 4.2.5. Sejam $\left(F, F^{\prime}\right) \hookrightarrow\left(E, E^{\prime}\right) \longrightarrow B$ um par fibrado e $\theta: \check{H}^{*}\left(F, F^{\prime}\right) \longrightarrow \check{H}^{*}\left(E, E^{\prime}\right)$ um $\mathbb{Z}_{2}$-homomorfismo tal que $\check{H}^{*}\left(F, F^{\prime}\right) \stackrel{\theta}{\longrightarrow} \check{H}^{*}\left(E, E^{\prime}\right) \longrightarrow \check{H}^{*}\left(F_{b}, F_{b}^{\prime}\right)$ é um isomorfismo, para todo $b \in B$. O homomorfismo $\theta$ é chamado extensão de cohomologia da fibra.

OBS 4.2.6. Dado um par fibrado em disco $\left(D^{n}, S^{n-1}\right) \hookrightarrow\left(E, E^{\prime}\right) \longrightarrow B$, uma extensão de 
cohomologia da fibra pode ser representada por uma classe $u \in \check{H}^{n}\left(E, E^{\prime}\right)$, no qual u restrita a cada par de fibras é um gerador.

Uma outra versão do teorema de Leray-Hirsch é dada a seguir.

Teorema 4.2.7. [2. Capítulo 7, Teorema 1.4, pg.372]. Dado um par fibrado $\left(F, F^{\prime}\right) \hookrightarrow\left(E, E^{\prime}\right) \stackrel{\pi}{\longrightarrow}$ $B$, no qual $F$ é paracompacto, $F^{\prime}$ é fechado e B é um complexo $C W$ finito, seja $\theta$ uma extensão de cohomologia da fibra. Então,

$$
\check{H}^{*}(B) \otimes \check{H}^{*}\left(F, F^{\prime}\right) \longrightarrow \check{H}^{*}\left(E, E^{\prime \prime}\right),
$$

definida por $b \otimes a \mapsto \pi^{*}(b) \cup \theta(a)$ é um isomorfismo de $\check{H}^{*}(B)$-módulos.

\subsection{As classes de Stiefel-Whitney}

Nesta seção, definiremos as classes características de Stiefel-Whitney, que são classes de cohomologia associadas a fibrados vetoriais.

Definição 4.3.1. Seja $\xi=\left\{E, \pi, B, \mathbb{R}^{n}\right\}$ um fibrado vetorial, no qual B é paracompacto. As classes de Stiefel-Whitney associadas à $\xi$ são classes $\omega_{i}(\xi) \in \check{H}^{i}\left(B, \mathbb{Z}_{2}\right)$ que satisfazem os seguintes axiomas:

$\left(S W_{1}\right) \omega_{0}(\xi)=1 e \omega_{i}(\xi)=0$ para $i>n$.

$\left(S W_{2}\right)$ Naturalidade. Se $f: B_{1} \longrightarrow$ B for uma função contínua, então $f^{*}\left(\omega_{i}(\xi)\right)=\omega_{i}\left(f^{*}(\xi)\right)$.

$\left(S W_{3}\right)$ Produto de Whitney. $\omega_{i}(\xi \oplus \eta)=\sum_{r+s=i} \omega_{r}(\xi) \cup \omega_{s}(\eta)$, no qual $\eta$ é fibrado vetorial sobre $B$. $\left(S W_{4}\right)$ Não trivialidade. $\omega_{1}\left(\gamma_{1}\right) \neq 0$, no qual $\gamma_{1}$ é o fibrado vetorial em linha canônico.

Seja $\xi=\left(E, \pi, B, \mathbb{R}^{n}\right)$ um fibrado vetorial, no qual $B$ é paracompacto. Consideremos o fibrado projetivo associado de $\xi, P(\xi)=\left(P(E), \pi^{\prime}, B, \mathbb{R} P^{n-1}\right)$, dado na Definição 1.6.3.

$\mathrm{O}$ espaço $\mathbb{R} P^{\infty}$ é a união $\cup_{m \in \mathbf{N}} \mathbb{R} P^{m}$ munida da topologia de Whitehead, isto é, um conjunto $A$ é fechado em $\mathbb{R} P^{\infty}$ se, e somente se, $A \cap \mathbb{R} P^{n}$ for fechado em $\mathbb{R} P^{n}$, para todo $n \in \mathbb{N}$.

Desde que $B$ é paracompacto, segue do Teorema 1.4.6, que existe uma aplicação de Gauss $g: E \longrightarrow \mathbb{R}^{\infty}$. Esta função induz uma função $P(g): P(E) \longrightarrow \mathbb{R} P^{\infty}$, definida por $P(g)[x]=$ 
$[g(x)]$. O diagrama 4.3.1 é comutativo.

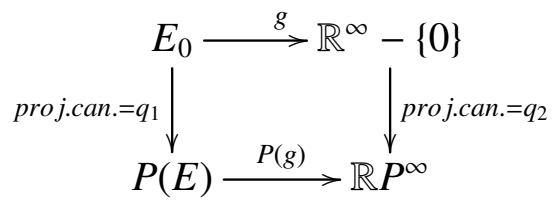

A função $P(g)$ está bem definida: como $g$ é linear e injetora nas fibras, então $[\mathrm{g}(\mathrm{x})]=[\mathrm{g}(\mathrm{y})]$ se, e somente se, $[\mathrm{x}]=[\mathrm{y}]$. A função $P(g)$ é contínua. De fato, seja $U$ aberto em $\mathbb{R} P^{\infty}$. Então, $P(g)^{-1}(U)$ é aberto em $P(E)$ se, e somente se, $q_{1}^{-1}\left(P(g)^{-1}(U)\right)$ for aberto em $E_{0}$. Como $g$ é contínua, segue da comutatividade do diagrama 4.3.1 que $q_{1}^{-1}\left(P(g)^{-1}(U)\right)=\left(P(g) \circ q_{1}\right)^{-1}(U)=$ $\left(q_{2} \circ g\right)^{-1}(U)$ é aberto em $E_{0}$.

A ideia é aplicar o teorema de Leray-Hirsch para o fibrado projetivo $\pi^{\prime}: P(E) \longrightarrow B$. Sejam $\alpha \in \check{H}^{1}\left(\mathbb{R} P^{\infty} ; \mathbb{Z}_{2}\right)$ um gerador [18, Exemplo 3.40, p. 250]. e $x=P(g)^{*}(\alpha) \in \check{H}^{1}\left(P(E) ; \mathbb{Z}_{2}\right)$. Consideremos uma injeção linear $\mathbb{R}^{n} \longrightarrow \mathbb{R}^{\infty}$, então podemos induzir um mergulho $\mathbb{R} P^{n-1} \longrightarrow$ $\mathbb{R} P^{\infty}$ tal que o diagrama abaixo é comutativo.

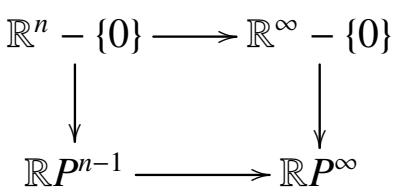

Então, $\alpha$ é levado em um gerador $\mathbf{a} \in \check{H}^{1}\left(\mathbb{R} P^{n-1} ; \mathbb{Z}_{2}\right)$ e cada $\alpha^{i}=\alpha \cup \cdots \cup \alpha \in H^{i}\left(\mathbb{R} P^{\infty}, \mathbb{Z}_{2}\right)$ é levado em um gerador $\mathbf{a}_{i} \in \check{H}^{i}\left(\mathbb{R} P^{n-1} ; \mathbb{Z}_{2}\right)$, para $i=1, \ldots, n-1$.

Note que quaisquer duas injeções lineares $i_{1}, i_{2}: \mathbb{R}^{n} \hookrightarrow \mathbb{R}^{\infty}$ são homotópicas através de injeções lineares e, então os mergulhos induzidos $j_{1}, j_{2}: \mathbb{R} P^{n-1} \longrightarrow \mathbb{R} P^{\infty}$ das fibras de $P(E)$ são homotópicos. De fato, $H:[0,1] \times \mathbb{R}^{n} \longrightarrow \mathbb{R}^{\infty}$ e $L:[0,1] \times \mathbb{R} P^{n-1} \longrightarrow \mathbb{R} P^{\infty}$, definidas por $H(t, v)=(1-t) i_{1}(v)+t i_{2}(v)$ e $L(t, v)=\left[(1-t) i_{1}(v)+t i_{2}(v)\right]$ são as homotopias procuradas entre $i_{1}, i_{2}$ e $j_{1}, j_{2}$, respectivamente.

Dado $b \in B, P(E)_{b}=\pi^{\prime-1}(b)$ é homeomorfo a $\mathbb{R} P^{n-1}$ e temos o seguinte diagrama:

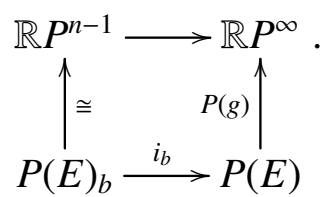


A aplicação da linha superior do diagrama 4.3.2 é homotópica à inclusão $j: \mathbb{R} P^{n-1} \hookrightarrow \mathbb{R} P^{\infty}$. Desde que $\mathbb{R} P^{n-1}$ é o $(n-1)$-esqueleto de $\mathbb{R} P^{\infty}$, então $j^{*}: \check{H}^{i}\left(\mathbb{R} P^{\infty}\right) \longrightarrow \check{H}^{i}\left(\mathbb{R} P^{n-1}\right)$ é um isomorfismo, para $i<n-1$ [28, Apêndice A, Corolário A.3, p. 261]. Logo, $\alpha$ é levado no gerador $i_{b}^{*}(x)=i_{b}^{*}\left(P(g)^{*} \alpha\right) \in \check{H}^{1}\left(P(E)_{b}\right)$. Analogamente, $i_{b}^{*}\left(x^{i}\right)=i_{b}^{*}\left(P(g)^{*}\left(\alpha^{i}\right)\right) \in \check{H}^{i}\left(P(E)_{b}\right)$ são geradores, para cada $i=0, \ldots, n-1$, no qual $x^{i}=x \cup x \cdots \cup x$.

OBS 4.3.2. [17], na prova do Teorema 1.16, p. 29 e 30, mostra que duas aplicações de Gauss $g_{1}, g_{2}: E \longrightarrow \mathbb{R}^{\infty}$, são homotópicas através de homotopias que são lineares e injetoras em cada fibra e, portanto, as classes $x^{i}$ independem da escolha da aplicação g.

Desde que, para todo $b \in B, \check{H}^{*}\left(P(E)_{b} ; \mathbb{Z}_{2}\right)$ é finitamente gerado, podemos aplicar o teorema de Leray-Hirsch (Teorema 4.2.1). Assim, $1, x, \ldots, x^{n-1}$ formam uma base para o $\check{H}^{*}(B)$-módulo $\check{H}^{*}(P(E))$. O elemento $x^{n}$ pode ser representado unicamente nesta base. Tem-se

$$
x^{n}+\omega_{1}(E) x^{n-1}+\cdots+\omega_{n}(E) 1=0,
$$

para certas classes $\omega_{i}(E) \in \check{H}^{*}(B)$.

OBS 4.3.3. Fazendo $\omega_{0}(E)=1$ e $\omega_{k}(E)=0$, para $k>n$, as classes $\omega_{i}(E)$ 's são candidatas às classes de Stiefel-Whitney de $\xi$.

Mostraremos a seguir que tais classes satisfazem os axiomas da Definição 4.3.1.

Proposição 4.3.4 (Naturalidade). $\omega_{i}\left(E_{1}\right)=f^{*}\left(\omega_{i}(E)\right)$, no qual $f: B_{1} \longrightarrow B$ é uma função contínua e $E_{1}$ é o espaço total de $f^{*}(\xi)$.

Demonstração: A função $f$ induz uma função $P(f): P\left(E_{1}\right) \longrightarrow P(E)$, que torna o seguinte diagrama comutativo.

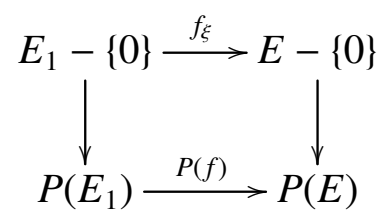

Então, temos $P(f)^{*}: \check{H}^{*}(P(E)) \longrightarrow \check{H}^{*}\left(P\left(E_{1}\right)\right)$, a qual leva a classe $x$ de $P(E)$ na classe $y$ de 
$P\left(E_{1}\right)$, nos quais $x$ e $y$ são obtidos como no procedimento visto anteriormente. Assim,

$$
\begin{aligned}
P(f)^{*}\left(\sum_{i} P(\pi)^{*}\left(\omega_{i}(E)\right) \cup x^{n-i}\right) & =\sum_{i} P(f)^{*} P(\pi)^{*}\left(\omega_{i}(E)\right) \cup P(f)^{*}\left(x^{n-i}\right) \\
& =\sum_{i} P\left(\pi_{1}\right)^{*}(f)^{*}\left(\omega_{i}(E)\right) \cup y^{n-i}
\end{aligned}
$$

pois o diagrama

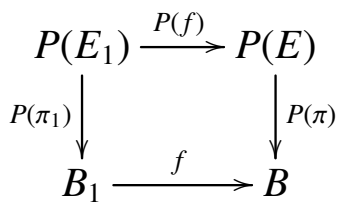

comuta. Portanto, $y^{n}+f^{*}\left(\omega_{1}(E)\right) y^{n-1}+\cdots+f^{*}\left(\omega_{n}(E)\right) 1=0$ e, assim, $\omega_{i}\left(E_{1}\right)=f^{*}\left(\omega_{i}(E)\right)$

Proposição 4.3.5 (Produto de Whitney). Sejam $\xi_{1}=\left(E_{1}, \pi_{1}, B, \mathbb{R}^{m}\right), \xi_{2}=\left(E_{2}, \pi_{2}, B, \mathbb{R}^{n}\right)$ fibrados vetoriais de dimensões $m$ e $n$, respectivamente. Então, $\omega\left(E_{1} \oplus E_{2}\right)=\omega\left(E_{1}\right) \cup \omega\left(E_{2}\right)$, no qual $\omega=1+\omega_{1}+\omega_{2}+\cdots \in \check{H}^{*}(B)$.

Demonstração: Seja $\xi_{1} \oplus \xi_{2}=\left(E_{1} \oplus E_{2}, q, B, \mathbb{R}^{m+n}\right)$. Podemos considerar $P\left(E_{1}\right)$ e $P\left(E_{2}\right)$ subconjuntos de $P\left(E_{1} \oplus E_{2}\right)$ da seguinte forma: uma classe de $P\left(E_{1} \oplus E_{2}\right)$ é da forma $[(x, y)]$, no qual $x \in \pi_{1}^{-1}(b)$ e $y \in \pi_{2}^{-1}(b)$ tais que $q(x, y)=\pi_{1}(x)=\pi_{2}(y)=b$. Os elementos $[x] \in P\left(E_{1}\right)$ estão relacionados com os elementos $\left[\left(x, 0_{\pi_{2}(x)}\right)\right] \in P\left(E_{1} \oplus E_{2}\right)$. Analogamente para os elementos de $P\left(E_{2}\right)$. A continuidade desta associação pode ser vista no diagrama a seguir.

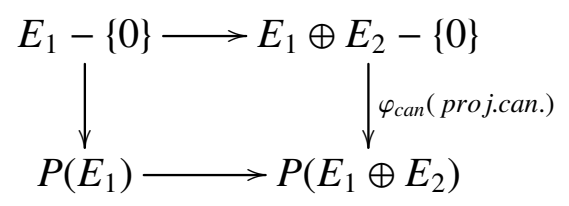

Os conjuntos $U_{1}=P\left(E_{1} \oplus E_{2}\right)-P\left(E_{1}\right)$ e $U_{2}=P\left(E_{1} \oplus E_{2}\right)-P\left(E_{2}\right)$ são abertos em $P\left(E_{1} \oplus E_{2}\right)$, pois $\varphi_{\text {can }}^{-1}\left(U_{1}\right)=\left\{(x, y) \in E_{1} \oplus E_{2}-\{0\} ; \pi_{1}(x)=\pi_{2}(y)\right.$ e $\left.\varphi_{\text {can }}(x, y) \notin P\left(E_{1}\right)\right\}=E_{1} \oplus E_{2}-\{0\} \cap$ $E_{1} \times E_{2}-\{0\}$ é aberto em $E_{1} \oplus E_{2}-\{0\}$. Analogamente para $U_{2}$. Os abertos $U_{1}$ e $U_{2}$ são retratos por deformação de $P\left(E_{2}\right)$ e $P\left(E_{1}\right)$, respectivamente. A aplicação $H: U_{1} \times I \longrightarrow U_{1}$, definida por $([x, y], t) \mapsto[(1-t) x, y]$ é a homotopia procurada. Analogamente para $U_{2}$. Seja 
$g: E_{1} \oplus E_{2} \longrightarrow \mathbb{R}^{\infty}$ uma aplicação de Gauss. Então, $g_{1}=\left.g\right|_{E_{1}}$ e $g_{2}=\left.g\right|_{E_{2}}$ são aplicações de Gauss (omitindo inclusões). Para o gerador $\alpha \in \check{H}^{1}\left(\mathbb{R} P^{\infty}\right)$, seja $P(g)^{*}(\alpha)=x \in \check{H}^{1}\left(P\left(E_{1} \oplus E_{2}\right)\right.$ ), no qual $P(g): P\left(E_{1} \oplus E_{2}\right) \longrightarrow \mathbb{R} P^{\infty}$ é induzida por $g$ (vista anteriormente). Note que $\left.P(g)\right|_{P\left(E_{1}\right)}=P\left(g_{1}\right)$ e $\left.P(g)\right|_{P\left(E_{2}\right)}=P\left(g_{2}\right)$. Então, $x$ se restringe às classes $P\left(g_{1}\right)^{*}(\alpha)$ e $P\left(g_{2}\right)^{*}(\alpha)$.

Consideremos as classes $\omega_{1}=\sum_{j} \omega_{j}\left(E_{1}\right) x^{m-j}$ e $\omega_{2}=\sum_{j} \omega_{j}\left(E_{2}\right) x^{n-j} \in \check{H}^{*}\left(P\left(E_{1} \oplus E_{2}\right)\right)$. Por definição, a classe $\omega_{1}$ se restringe a zero em $\check{H}^{m}\left(P\left(E_{1}\right)\right)$. Considerando a sequência exata do par $\cdots \rightarrow \check{H}^{m}\left(P\left(E_{1} \oplus E_{2}\right), P\left(E_{1}\right)\right) \cong \check{H}^{m}\left(P\left(E_{1} \oplus E_{2}\right), U_{2}\right) \stackrel{j_{1}^{*}}{\rightarrow} \check{H}^{m}\left(P\left(E_{1} \oplus E_{2}\right)\right) \rightarrow \check{H}^{m}\left(P\left(E_{1}\right)\right) \rightarrow \cdots$

existe $j_{1}^{*}\left(a_{1}\right)=x$. Analogamente, para $\omega_{2}$. Temos o seguinte diagrama comutativo

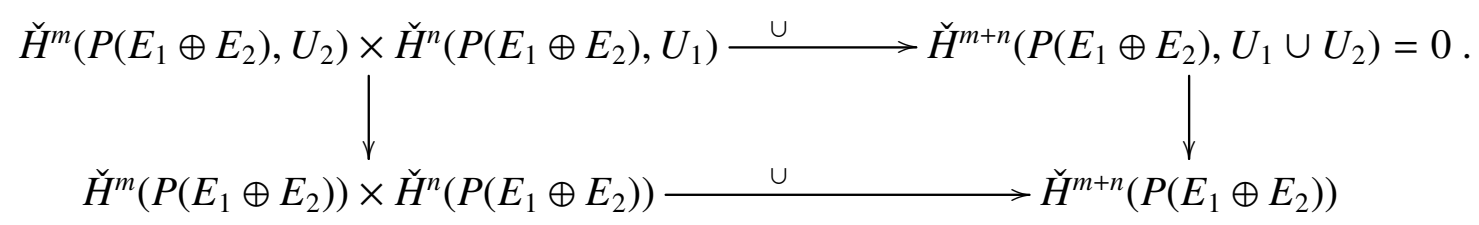

Então, $\omega_{1} \omega_{2}=\sum_{j}\left[\sum_{r+s=j} \omega_{r}\left(E_{1}\right) \omega_{s}\left(E_{2}\right)\right] x^{m+n-j}=0$, que é a definição da relação para as classes de Stiefel-Whitney para $E_{1} \oplus E_{2}$. Assim, $\omega_{j}\left(E_{1} \oplus E_{2}\right)=\sum_{r+s=j} \omega_{r}\left(E_{1}\right) \omega_{s}\left(E_{2}\right)$.

Proposição 4.3.6. $\omega_{i}(E)=0$, para $i>n$;

Demonstração: Segue imediatamente da definição.

Proposição 4.3.7 (Não trivialidade). Para o fibrado em linha canônico $E\left(\gamma^{1}\right) \longrightarrow G_{1}=\mathbb{R} P^{\infty}$, $\omega_{1}(E) \in \check{H}^{1}\left(G_{1}\right)=\check{H}^{1}\left(\mathbb{R} P^{\infty}\right)$ é um gerador.

Demonstração: A projeção $\pi_{1}: E\left(\gamma^{1}\right) \longrightarrow \mathbb{R} P^{\infty}$ induz a aplicação $P\left(\pi_{1}\right): P(E) \longrightarrow \mathbb{R} P^{\infty}$. As classes de $P(E)$ são da forma $[([v], x)]$, no qual $x \in[v]$, isto é, $x=\lambda v(\lambda \in \mathbb{R})$, logo $[v]$ determina unicamente uma classe em $P(E)$, pois $[([v], x)]$ é a classe dos múltiplos de $([v], x)$ que são todos os elementos $([\mathrm{v}], \mathrm{y})$ tais que $y \in[v]$. Podemos pensar em $P\left(\pi_{1}\right)$ como sendo uma identidade.

A aplicação de Gauss $g: E \longrightarrow \mathbb{R}^{\infty}$ induz a aplicação $P(g): P(E) \longrightarrow \mathbb{R} P^{\infty}$, que também pode ser considerado como uma identidade. Dado um gerador $\alpha \in \check{H}^{1}\left(\mathbb{R} P^{\infty}\right)$, então $x=P(g)^{*}(\alpha) \in \check{H}^{1}(P(E)) \cong \check{H}^{1}\left(\mathbb{R} P^{\infty}\right)$ é um gerador. Como $x+\omega_{1}(E) 1=0$, segue que $\omega_{1}(E) \in \check{H}^{1}\left(\mathbb{R} P^{\infty}\right)$ é um gerador. 


\subsection{Unicidade das classes de Stiefel-Whitney}

Na seção anterior, construímos certas classes satisfazendo os axiomas da Definição 4.3.1. Agora, para concluirmos que tais classes são as classes de Stiefel-Whitney basta verificarmos a sua unicidade.

Definição 4.4.1. Seja $\xi=\left(E, \pi, B, \mathbb{R}^{n}\right)$ um fibrado vetorial. Uma aplicação fatoração de $\xi$ é um função contínua $f: B_{1} \longrightarrow B$ tal que $f^{*}(\xi)$ se escreve como uma fatoração $\lambda_{1} \oplus \cdots \oplus \lambda_{n}$, no qual cada $\lambda_{i}$ é um fibrado vetorial em linha, e $f^{*}: \check{H}^{*}(B) \longrightarrow \check{H}^{*}\left(B_{1}\right)$ é injetora.

Proposição 4.4.2. Todo fibrado vetorial $\xi=\left(E, \pi, B, \mathbb{R}^{n}\right)$ com espaço base paracompacto $B$ possui uma aplicação fatoração.

Demonstração: A demonstração será feita por indução sobre a dimensão do fibrado vetorial. Se $\xi$ for um fibrado vetorial em linha, isto é, se sua dimensão for $n=1$, então a função identidade é uma aplicação fatoração para $\xi$. Suponhamos que o resultado seja válido para fibrados vetoriais com dimensão menor que $n$. Seja $P(\xi)$ o fibrado projetivo associado de $\xi$. Consideremos o fibrado vetorial $P(\pi)^{*}(\xi)$, no qual $P(\pi): P(E) \longrightarrow B$. Este fibrado contém o subfibrado vetorial em linha com espaço total $L=\left\{([v], x) \in E\left(P(\pi)^{*}(\xi)\right) ; x \in[v]\right\}$ dos pontos $(l, x)$, no qual $l$ é uma reta e $x \in l$. Como $P(\pi)^{*}(\xi)$ possui métrica, este pode ser fatorado em $\lambda_{1} \oplus \lambda_{1}^{\perp}$. Desde que $\lambda_{1}^{\perp}$ possui dimensão $n-1$, segue por hipótese de indução que existe $g: B_{1} \longrightarrow P(E)$, aplicação fatoração para $\lambda_{1}^{\perp}$. Sendo $P(\pi)^{*}$ injetora, temos $f=P(\pi) \circ g: B_{1} \longrightarrow B$ uma aplicação fatoração para $\xi$

Teorema 4.4.3. As classes de Stiefel-Whitney são unicamente determinadas pelos axiomas $S W_{1}$ a $S W_{4}$ dados na Definição 4.3.1

Demonstração: Suponhamos que $\omega_{i}(E)$ e $\bar{\omega}_{i}(E)$ sejam classes de Stiefel-Whitney para um fibrado vetorial $\xi=\left(E, \pi, B, \mathbb{R}^{n}\right)$ com espaço base $B$ paracompacto e seja $f: B_{1} \rightarrow B$ aplicação fatoração de $\xi$. Como a primeira classe de Stiefel-Whitney é unicamente determinada para fibrados vetoriais em linha $\lambda_{i}$, temos $\omega_{1}\left(\lambda_{i}\right)=\bar{\omega}_{1}\left(\lambda_{i}\right)$, para todo $i=1, \cdots, n$, no qual $f^{*}(\xi)$ é $B$ isomorfo a $\lambda_{1} \oplus \cdots \oplus \lambda_{n}$. Sejam $E_{1}$ o espaço total de $f^{*}(\xi)$ e $\omega(E)=1+\omega_{1}(E)+\cdots+\omega_{n}(E), \bar{\omega}(E)=$ $1+\bar{\omega}_{1}(E)+\cdots+\bar{\omega}_{n}(E) \in \check{H}^{*}(B)$. Assim, 


$$
\begin{aligned}
f^{*}(\omega(E)) & =\omega\left(E_{1}\right)=\left(1+\omega_{1}\left(\lambda_{1}\right)\right) \cdots\left(1+\omega_{1}\left(\lambda_{n}\right)\right) \\
& =\left(1+\bar{\omega}_{1}\left(\lambda_{1}\right)\right) \cdots\left(1+\bar{\omega}_{1}\left(\lambda_{n}\right)\right) \\
& =\bar{\omega}\left(E_{1}\right)=f^{*}(\bar{\omega}(E)) .
\end{aligned}
$$

Desde que $f^{*}$ é injetora, segue que $\omega(E)=\bar{\omega}(E)$.

Assim as classes $\omega_{i}(E)$ são as classes de Stiefel-Whitney $\omega_{i}(\xi)$ do fibrado vetorial $\xi=$ $\left(E, \pi, B, \mathbb{R}^{n}\right)$. Usaremos ambas as notações $\omega_{i}(E)$ ou $\omega_{i}(\xi)$ para nos referirmos a $i$-ésima classe de Stiefel-Whitney associado ao fibrado vetorial $\xi=\left(E, \pi, B, \mathbb{R}^{n}\right)$.

\subsection{A classe característica de Euler}

Um elemento $c \in \check{H}^{n}\left(E, E^{\prime} ; R\right)$, cuja restrição a cada fibra $\left(D^{n}, S^{n-1}\right)$ é um gerador de $\check{H}^{n}\left(D^{n}, S^{n-1} ; R\right)$ é chamado uma classe de Thom para o par fibrado $\left(D^{n}, S^{n-1}\right) \hookrightarrow\left(E, E^{\prime}\right) \longrightarrow B$. Tal elemento, quando a cohomologia possui coeficientes em $\mathbb{Z}_{2}$, coincide com o representante de uma extensão de cohomologia da fibra ${ }^{4}$

É fato que todo fibrado em disco possui uma classe de Thom com coeficientes em $\mathbb{Z}_{2}[18$, Capítulo 4, Teorema 4D.10, p. 440] ou, analogamente, todo fibrado em disco possui uma extensão de cohomologia da fibra [33, Capítulo 5, p. 259].

Teorema 4.5.1 (Teorema do Isomorfismo de Thom). Seja $\left(D^{n}, S^{n-1}\right) \hookrightarrow\left(E, E^{\prime}\right) \longrightarrow B$ um fibrado em disco. Então, a aplicação $\phi: \check{H}^{i}\left(B ; \mathbb{Z}_{2}\right) \longrightarrow \check{H}^{i+n}\left(E, E^{\prime} ; \mathbb{Z}_{2}\right)$ definida por $\phi(b)=$ $\pi^{*}(b) \cup$ c é um isomorfismo, para todo $i \geq 0$ e $\check{H}^{i}\left(E, E^{\prime} ; \mathbb{Z}_{2}\right)=0$, para $i<n$. O isomorfismo $\phi e ́$ chamado isomorfismo de Thom.

Demonstração: A aplicação definida por $\theta(z)=c$, no qual $z \in \check{H}^{n}\left(D^{n}, S^{n-1} ; \mathbb{Z}_{2}\right)$ é o elemento não nulo, define uma extensão de cohomologia da fibra. Pelo teorema de Leray-Hirsch 4.2.7, existe um isomorfismo $\check{H}^{i}\left(B ; \mathbb{Z}_{2}\right) \cong \check{H}^{i}\left(B ; \mathbb{Z}_{2}\right) \otimes \check{H}^{n}\left(D^{n}, S^{n-1} ; \mathbb{Z}_{2}\right) \longrightarrow \check{H}^{i+n}\left(E, E^{\prime} ; \mathbb{Z}_{2}\right)$, definido por, $b \mapsto b \otimes z \mapsto \pi^{*}(b) \cup c$.

\footnotetext{
${ }^{4}$ Vide Definição 4.2 .5
} 
Seja $f: X \longrightarrow Y$ uma função contínua, o mapping cylinder $M_{f}$ é o espaço quociente da união disjunta $(X \times[0,1]) \bigsqcup Y$ obtida por identificar cada $(x, 1) \in X \times[0,1] \operatorname{com} f(x) \in Y$. Verifica-se que um mapping cylinder $M_{f}$ é um retrato por deformação sobre $Y$ [18, p.2].

Dado um fibrado em esfera $S^{n-1} \hookrightarrow E \stackrel{\pi}{\longrightarrow} B$, então $D^{n} \hookrightarrow M_{\pi} \stackrel{\pi}{\longrightarrow} B$ é um fibrado em disco e temos o par fibrado $\left(D^{n}, S^{n-1}\right) \hookrightarrow\left(M_{\pi}, E\right) \stackrel{\pi}{\longrightarrow} B$. Consideremos o seguinte diagrama comutativo,

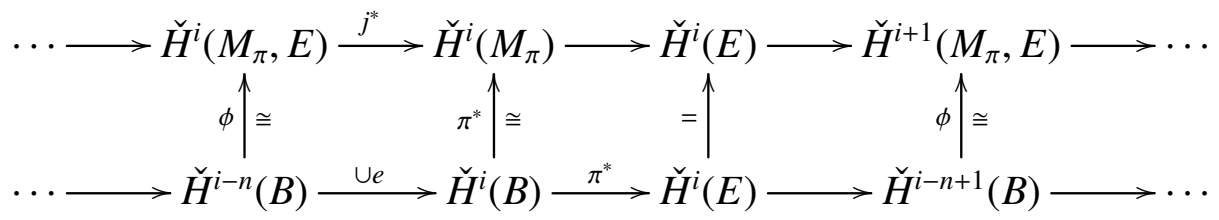

no qual a sequência superior é a sequência exata longa do par $\left(M_{\pi}, E\right)$. Os isomorfismos $\phi$ são isomorfismos de Thom e a aplicação vertical $\pi^{*}$ é um isomorfismo, pois $M_{\pi}$ é um retrato por deformação sobre $B$. A sequência inferior é uma sequência exata chamada sequência de Gysin do fibrado em esfera $S^{n-1} \hookrightarrow E \longrightarrow B$.

Definição 4.5.2. A classe $e \in \check{H}^{n}\left(B ; \mathbb{Z}_{2}\right)$ definida como $e=\left(\pi^{*}\right)^{-1} \circ j^{*}(c)$, no qual $c \in \check{H}^{n}\left(M_{\pi}, E\right)$ é uma classe de Thom, é chamada classe caractenística de Euler do fibrado em esfera $S^{n-1} \hookrightarrow$ $E \stackrel{\pi}{\longrightarrow} B$

OBS 4.5.3. Observemos que se $X$ for um $\mathbb{Z}_{2}$-espaço livre, a projeção canônica $X \longrightarrow X \bmod \mathbb{Z}_{2}$ é, então um fibrado em esfera. Dado outro $\mathbb{Z}_{2}$-espaço livre $Y$, se $f: X \longrightarrow Y$ for uma aplicação $\mathbb{Z}_{2}$-equivariante e $\bar{f}: X \bmod \mathbb{Z}_{2} \longrightarrow Y \bmod \mathbb{Z}_{2}$ for a induzida por $f$, temos o seguinte diagrama comutativo.

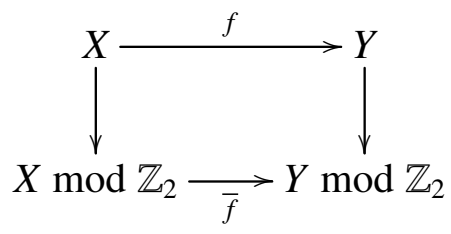

Também, $\bar{f}$ leva classe de Euler em classe de Euler. 


\section{Capítulo}

\section{5}

\section{Versão parametrizada do teorema de}

\section{Borsuk-Ulam}

O teorema clássico de Borsuk-Ulam afirma que toda função contínua $f: S^{n} \longrightarrow \mathbb{R}^{n}$ colapsa pelo menos um par de pontos antípodas, isto é, existem $x,-x \in S^{n}$ tais que $f(x)=f(-x)$.

Nesse capítulo, apresentaremos uma demonstração de uma versão deste teorema conhecida como Versão parametrizada do teorema de Borsuk-Ulam provada por A. Dold em [9]. Esta é uma versão do teorema no contexto de fibrados. Considerando dois fibrados vetoriais $\xi=$ $\left(E, \pi, B, \mathbb{R}^{m}\right)$ e $\xi^{\prime}=\left(E^{\prime}, \pi^{\prime}, B, \mathbb{R}^{n}\right)$ com mesmo espaço base $B$ paracompacto e uma aplicação $\mathbb{Z}_{2}$-equivariante $f: S(E) \longrightarrow E^{\prime}$ que preserva fibras (isto é, $\pi^{\prime} \circ f=\pi_{\S(E)}$ ), no qual $S(E)$ denota o espaço total do fibrado em esfera associado de $\xi$, a versão parametrizada do teorema de Borsuk-Ulam consiste em estimar a dimensão cohomológica do conjunto

$$
\overline{Z_{f}}=\{[x] \in \overline{S(E)} ; \bar{f}([x])=0\},
$$

no qual $\overline{S(E)}$ é o conjunto de órbitas de $S(E)$ pela ação antipodal de $\mathbb{Z}_{2}$ e $\bar{f}$ é a aplicação induzida por esta ação. Mais especificamente,

$$
\operatorname{dim}_{\mathrm{c}} \overline{Z_{f}} \geq \operatorname{dim}_{\mathrm{c}} B+m-n-1
$$


no qual $\operatorname{dim}_{\mathrm{c}}$ significa dimensão cohomológica ${ }^{1}$

\subsection{Versão parametrizada do teorema de Borsuk-Ulam para fibrados em esfera}

Sejam $\xi=\left(E, \pi, B, \mathbb{R}^{m}\right)$ e $\xi^{\prime}=\left(E^{\prime}, \pi^{\prime}, B, \mathbb{R}^{n}\right)$ dois fibrados vetoriais reais, no qual $B$ é um espaço paracompacto. Denotaremos por $\omega_{i}(E)$ e $\omega_{i}\left(E^{\prime}\right)$ as classes de Stiefel-Whitney (Definição 4.3.1) de $\xi$ e $\xi^{\prime}$, respectivamente.

Definição 5.1.1. O polinômio $\omega(E, t)=\sum_{j=0}^{m} \omega_{j}(E) t^{m-j} \in \check{H}^{*}(B)[t]$ é chamado de polinômio de Stiefel-Whitney de $\xi$. De maneira análoga, definimos $\omega\left(E^{\prime}, t\right)=\sum_{j=0}^{n} \omega_{j}\left(E^{\prime}\right) t^{n-j} \in \check{H}^{*}(B)[t]$.

Seja $S(E)$ o espaço total do fibrado em esfera associado à $\xi$ (Definição 1.6.2). Considere a ação antipodal

$$
\begin{aligned}
\mathrm{S}(\mathrm{E}) \times \mathbb{Z}_{2} & \longrightarrow \mathrm{S}(\mathrm{E}) \\
( \pm 1, x) & \longmapsto \pm x .
\end{aligned}
$$

Denotaremos o espaço de órbitas de $S(E)$ pela ação acima por $\overline{S(E)}$. A projeção canônica $p: S(E) \longrightarrow \overline{S(E)}$ nos dá um $\mathbb{Z}_{2}$-fibrado principal. Além disso, $p: S(E) \longrightarrow \overline{S(E)}$ é um recobrimento a 2-folhas.

Agora, seja $h: S(E) \longrightarrow E^{\prime}$ uma função $\mathbb{Z}_{2}$-equivariante preservando fibras, ou seja, $h(-x)=-h(x)$ e $\pi^{\prime} \circ h=\left.\pi\right|_{S(E)}$. Seja $Z=\{x \in S(E) ; h(x)=0\}$. Note que $Z$ também é um $\mathbb{Z}_{2}$-espaço livre. A aplicação $p$ quando restrita a $Z$ também nos fornece um $\mathbb{Z}_{2}$-fibrado principal e um recobrimento a 2-folhas $\left.p\right|_{Z}: Z \longrightarrow \bar{Z}$.

Interpretando $\mathbb{Z}_{2}$ como uma 0 -esfera $S^{0}$, podemos associar as classes características de Euler (Definição 4.5.2) aos $\mathbb{Z}_{2}$-fibrados principais $p: S(E) \longrightarrow \overline{S(E)}$ e $\left.p\right|_{Z}: Z \longrightarrow \bar{Z}$. Sejam $u \in$ $\check{H}^{1}(\overline{S(E)})$ e $\left.u\right|_{Z} \in \check{H}^{1}(\bar{Z})$ tais classes. Temos os seguintes homomorfismos de $\check{H}^{*}(B)$ - álgebras:

$$
\sigma: \check{H}^{*}(B)[t] \quad \longrightarrow \check{H}^{*}(\overline{S(E)})
$$

${ }^{1}$ Vide Definição 5.1 .3 
e compondo com a induzida da inclusão $i: \check{H}^{*}(\overline{S(E)}) \longrightarrow \check{H}^{*}(\bar{Z})$ temos

$$
\begin{aligned}
\left.\sigma\right|_{\bar{Z}}: \check{H}^{*}(B)[t] & \longrightarrow \check{H}^{*}(\bar{Z}) \\
t & \left.\longmapsto u\right|_{\bar{Z}} .
\end{aligned}
$$

Denotaremos por $\left.p(t)\right|_{\bar{Z}}$ a imagem de um polinômio $p(t)$ pelo homomorfismo $\left.\sigma\right|_{\bar{Z}}$. Nestas condições temos o seguinte teorema.

Teorema 5.1.2. Se $q(t) \in \check{H}^{*}(B)[t]$ é tal que $\left.q(t)\right|_{\bar{Z}}=0$, então $q(t) \omega\left(E^{\prime} ; t\right)=\omega(E ; t) q^{\prime}(t)$, para algum polinômio $q^{\prime}(t) \in \check{H}^{*}(B)[t]$.

Provaremos uma generalização deste teorema adiante. Agora iremos ver algumas de suas consequências.

Suponhamos $m>n$. Se o grau de $q(t)$ for menor que $m-n$, então $q(t) \omega\left(E^{\prime} ; t\right)$ terá grau menor que $m$. Logo, não existirá $q^{\prime}(t)$ tal que $q(t) \omega\left(E^{\prime} ; t\right)=\omega(E ; t) q^{\prime}(t)$, pois $\omega(E ; t)$ já possui grau $m$. Assim, $\left.q(t)\right|_{\bar{Z}} \neq 0$. Então, o $\check{H}^{*}(B)$-homomorfismo

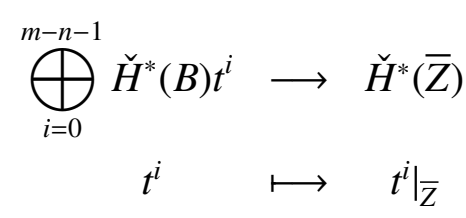

possui núcleo trivial e portanto é um monomorfismo.

Definição 5.1.3. [14. Capítulo 1, p. 95]. Seja X um espaço topológico. A dimensão cohomológica de X é um valor inteiro denotado por $\operatorname{dim}_{\mathrm{c}} X$ e definido da seguinte maneira:

(i) $\operatorname{dim}_{\mathrm{c}} X=-1$ se, e somente se, $X=\emptyset$;

(ii) $\operatorname{dim}_{\mathrm{c}} X \leq n$, no qual $n=0,1,2, \ldots$, se $\check{H}^{n+i}(X, F)=0$, para todo $F \subset X$ fechado $e$ $i=1,2, \ldots$;

(iii) $\operatorname{dim}_{\mathrm{c}} X=n$ se $\operatorname{dim}_{\mathrm{c}} X \leq n e \operatorname{dim}_{\mathrm{c}} X>n-1$;

(iv) $\operatorname{dim}_{\mathrm{c}} X=\infty$ se $\operatorname{dim}_{\mathrm{c}} X \geq n$, para todo $n=0,1,2, \ldots$

No caso em que $m>n$ e $\operatorname{dim}_{\mathrm{c}} B$ finita, dado $\alpha \in \check{H}^{\operatorname{dim}_{\mathrm{c}} B}(B)$, temos $\left.\alpha t^{i} \mapsto \alpha u\right|_{\bar{Z}} ^{i} \neq 0$, para $i<m-n . \operatorname{Logo}, \operatorname{dim}_{\mathrm{c}} \bar{Z} \geq \operatorname{dim}_{\mathrm{c}} B+m-n-1$. Se $\operatorname{dim}_{\mathrm{c}} B=\infty$, também teremos $\operatorname{dim}_{\mathrm{c}} \bar{Z}=\infty$. 
Assim, obtemos o seguinte corolário, o qual é uma versão parametrizada do teorema clássico de Borsuk-Ulam.

Corolário 5.1.4. Se $m>n$, todos os polinômios $q(t)$ de grau, com respeito à $t$, menor que $m-n$ satisfazem $\left.q(t)\right|_{\bar{Z}} \neq 0$. Em outras palavras, o $\check{H}^{*}(B)$-homomorfismo

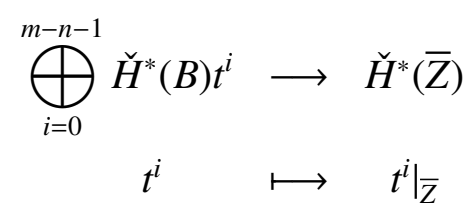

é injetor. Em particular,

$$
\operatorname{dim}_{\mathrm{c}} \bar{Z} \geq \operatorname{dim}_{\mathrm{c}} B+m-n-1 \text {. }
$$

Como caso particular do Corolário 5.1.4, considerando $B$ como sendo um conjunto com um único ponto, podemos provar o teorema de Borsuk-Ulam dado por Yang em [37].

Teorema 5.1.5 (Borsuk-Ulam). Se $f: S^{m} \longrightarrow \mathbb{R}^{n}$ é uma função contínua e $m \geq n$, então existe $x \in S^{m}$ tal que $f(x)=f(-x)$.

Demonstração: Considere o caso em que $B=\{b\}$ e $\operatorname{dim} \xi=m+1$. Então, $E \cong \mathbb{R}^{m+1}, E^{\prime} \cong \mathbb{R}^{n}$ e $S(E) \cong S^{m}$.

Seja $g: S(E) \longrightarrow E^{\prime}$ uma função contínua. Neste caso, já temos $\pi^{\prime} \circ g=\pi$. Defina $h: S(E) \longrightarrow E^{\prime}$ por $h(x)=g(x)-g(-x)$. Então, $h$ é uma função contínua tal que $\pi^{\prime} \circ h=\pi$ e $h(-x)=g(-x)-g(x)=-h(x)$. Denotando por $A(g)$ o conjunto dos pontos de coincidência antipodal da função $g$, temos

$$
A(g)=\{x \in S(E) ; g(-x)=g(x)\}=\{x \in S(E) ; h(x)=0\}=Z .
$$

Então, do Corolário 5.1.4

$$
\operatorname{dim}_{\mathrm{c}} \overline{A(g)}=\operatorname{dim}_{\mathrm{c}} \bar{Z} \geq \operatorname{dim}_{\mathrm{c}} B+m+1-n-1=m-n \geq 0 .
$$

Logo, $\overline{A(g)} \neq \emptyset$. Portanto, $A(g) \neq \emptyset$.

Dada $f: S^{m} \longrightarrow \mathbb{R}^{n}$ contínua, obtemos $g: S(E) \longrightarrow E^{\prime}$ como acima que torna o seguinte diagrama comutativo. 


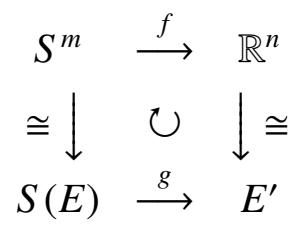

Logo, existe um ponto $x \in S^{m}$ tal que $f(x)=f(-x)$.

No caso particular, em que $m=n+1$, obtemos o teorema clássico de Borsuk-Ulam.

\subsection{Versão Cohomológica}

Definição 5.2.1. Um espaço topológico $X$ tem dimensão finita menor ou igual a $n(\operatorname{dim} X \leq n)$ se toda cobertura aberta de $X$ possui um refinamento cujo nervo é um complexo simplicial de dimensão $\leq n$. Se $\operatorname{dim} X \leq n e \operatorname{dim} X \not \leq n-1$, então $X$ tem dimensão $n$, denotado por $\operatorname{dim} X=n$. Se $\operatorname{dim} X \not \leq n$, para todo natural $n$, então $\operatorname{dim} X=\infty$.

Uma maneira equivalente de definir dimensão de espaços topológicos é dado a seguir.

Definição 5.2.2. Uma coleção $\mathcal{A}$ de subconjunto do espaço $X$ é dita de ordem $m+1$ se algum ponto de $X$ está em $m+1$ elementos de $\mathcal{A}$, e nenhum ponto de $X$ está em mais do que $m+1$ elementos de $\mathcal{A}$.

Definição 5.2.3. Um espaço topológico $X$ é dito ser de dimensão finita se existe algum inteiro positivo $m$ tal que para cada cobertura aberta $\mathcal{U}$ de $X$, existe um refinamento $V$ que tem ordem no máximo $m+1$. A dimensão topológica de $X$ é definida como o menor valor de m para o qual o enunciado vale.

Definição 5.2.4. Sejam $S, B, Y$ e $G$ espaços paracompactos, no qual $G$ é um grupo topológico. $U m G$-fibrado em esfera de dimensão $m-1$ é uma aplicação $\pi: S \longrightarrow$ B juntamente com uma G-ação $\tau$ livre nas fibras $\pi^{-1}(b) \cong Y$ em $S$ tal que

i) $(\pi, \tau)$ é G-localmente trivial, isto é, existe uma cobertura aberta $\left\{U_{\alpha}\right\}$ de B, no qual para cada $U_{\alpha}$, existe um homeomorfismo $h_{\alpha}: U_{\alpha} \times Y \longrightarrow \pi^{-1}\left(U_{\alpha}\right) G$-equivariante, no qual a ação em $U_{\alpha} \times Y$ é dada por $(u, y) g=(u, \tau(g, y))$, para todo $(u, y) \in U_{\alpha} \times Y$ e $g \in G$;

ii) A fibra Y é compacta de dimensão finita; 
iii) $\check{H}^{*}(Y) \cong \check{H}^{*}\left(S^{m-1}\right)$.

De (ii) a fibra $Y$ tem dimensão finita. Seja $p: Y \longrightarrow \bar{Y}$ a projeção canônica.

Seja $\overline{\mathcal{U}}$ uma cobertura aberta para $\bar{Y}$, então $\mathcal{U}=p^{-1}(\overline{\mathcal{U}})$ (conjunto formado pelas imagens inversas dos abertos em $\overline{\mathcal{U}}$ por $p$ ) é uma cobertura aberta para $Y$. Logo existe um refinamento $\mathcal{V}$ de $\mathcal{U}$ com ordem finita. Seja $\overline{\mathcal{V}}$ a imagem de $\mathcal{V}$ por $p$. Então, $\overline{\mathcal{V}}$ é um refinamento de $\overline{\mathcal{U}}$ com ordem finita. Então, $\bar{Y}$ tem dimensão finita.

Usando a Definição 5.2.1 de dimensão para espaços topológicos, vemos que $\check{H}^{j}(\bar{Y})=0$, para $j$ grande.

Seja $u_{Y} \in \check{H}^{1}(\bar{Y})$ a classe de Euler do fibrado em esfera $S^{0} \hookrightarrow Y \stackrel{p}{\longrightarrow} \bar{Y}$ e consideremos a sequência de Gysin associada a este fibrado (Vide Seção 4.5).

$$
\begin{gathered}
\check{H}^{0}(\bar{Y}) \stackrel{p^{*}}{\longrightarrow} \check{H}^{0}(Y) \stackrel{\tau}{\longrightarrow} \check{H}^{0}(\bar{Y}) \stackrel{\cup u_{Y}}{\longrightarrow} \check{H}^{1}(\bar{Y}) \stackrel{p^{*}}{\longrightarrow} \check{H}^{1}(Y) \stackrel{\tau}{\longrightarrow} \check{H}^{1}(\bar{Y}) \stackrel{\cup u_{Y}}{\longrightarrow} \check{H}^{2}(\bar{Y}) \stackrel{p^{*}}{\rightarrow} \check{H}^{2}(Y) \rightarrow \cdots \\
\cdots \longrightarrow \check{H}^{m-2}(Y) \stackrel{\tau}{\rightarrow} \check{H}^{m-2}(\bar{Y}) \stackrel{\cup u_{Y}}{\longrightarrow} \check{H}^{m-1}(\bar{Y}) \stackrel{p^{*}}{\rightarrow} \check{H}^{m-1}(Y) \stackrel{\tau}{\rightarrow} \check{H}^{m-1}(\bar{Y}) \stackrel{\cup u_{Y}}{\longrightarrow} \check{H}^{m}(\bar{Y}) \stackrel{p^{*}}{\rightarrow} \check{H}^{m}(Y) \longrightarrow \\
\check{H}^{m}(\bar{Y}) \stackrel{\cup u_{Y}}{\longrightarrow} \check{H}^{m+1}(\bar{Y}) \stackrel{p^{*}}{\rightarrow} \check{H}^{m+1}(Y) \stackrel{\tau}{\rightarrow} \check{H}^{m+1}(\bar{Y}) \longrightarrow \cdots
\end{gathered}
$$

Como $Y$ é conexo e a cohomologia de Čech na dimensão zero nos fornece o número de componentes conexas do espaço, $\check{H}^{0}(Y)=\mathbb{Z}_{2}$ e $p^{*}: \check{H}^{0}(\bar{Y}) \rightarrow \check{H}^{0}(Y)$ é um isomorfismo.

Assim $\cup u_{Y}: \check{H}^{0}(\bar{Y}) \rightarrow \check{H}^{1}(\bar{Y})$ é injetor, logo $u_{Y}=1 \cup u_{Y}$ é uma classe não nula. O fato que $\check{H}^{i}(Y)=0$ para $1 \leq i \leq m-2$ implica que $\cup u_{Y}: \check{H}^{i}(\bar{Y}) \rightarrow \check{H}^{i}(\bar{Y})$ é um isomorfismo para $0 \leq i \leq m-2$ e injetor para $i=m-1$, consequentemente, cada potência $u_{Y}^{i}$ gera $\check{H}^{i}(\bar{Y}) \cong \mathbb{Z}_{2}$ para $0 \leq i \leq m-2$ e $u_{Y}^{m-1} \in \check{H}^{m-1}(\bar{Y})$ é uma classe não nula. Mais ainda, vemos que $\check{H}^{j}(\bar{Y})=0$ para $j \geq m$ e, portanto, $u_{Y}^{j}=0$ para $j \geq m$. Agora, como $\check{H}^{m}(\bar{Y})=0$, segue da exatidão da sequência de Gysin que $\check{H}^{m-1}(Y) / \operatorname{ker} \tau \cong \check{H}^{m-1}(\bar{Y})$ e, como $\check{H}^{m-1}(Y) \cong \mathbb{Z}_{2}$ concluímos que $\check{H}^{m-1}(\bar{Y}) \cong \mathbb{Z}_{2}$ com gerador $u_{Y}^{m-1}$, pois caso contrário, se $\operatorname{ker} \tau=\check{H}^{m-1}(Y)$ teríamos $\breve{H}^{m-1}(\bar{Y})=0$, o que é uma contradição. Deste modo, o anel de cohomologia $\check{H}^{*}(\bar{Y})$ é dado por

$$
\check{H}^{*}(\bar{Y}) \cong \frac{\mathbb{Z}_{2}\left[u_{Y}\right]}{\left(u_{Y}^{m}\right)}
$$


A aplicação $Y \hookrightarrow S \stackrel{\pi}{\longrightarrow} B$ induz $\bar{Y} \hookrightarrow \bar{S} \stackrel{\bar{\pi}}{\longrightarrow} B$. Dado $b \in B$, temos $F_{b}=\bar{\pi}^{-1}(b) \cong \bar{Y} \mathrm{e}$ $\check{H}^{*}\left(F_{b}\right) \cong \frac{\mathbb{Z}_{2}\left[u_{b}\right]}{\left(u_{b}^{m}\right)}$. Seja $u \in \check{H}^{1}(\bar{S})$ a classe de Euler de $S \longrightarrow \bar{S}$.

Como $F_{b} \hookrightarrow \bar{S}$, temos $\left.u \mapsto u\right|_{F_{b}}=u_{b}$. Como $\check{H}^{*}\left(F_{b}\right)$ é gerado por $1, u_{b}, u_{b}^{2}, \ldots, u_{b}^{m-1}$, as classes $u^{i} \in \check{H}^{i}(\bar{S})$ são tais que $\left.u^{i}\right|_{F_{b}}=u_{b}^{i}$. Também, como $\check{H}^{j}\left(F_{b}\right)$ são finitamente gerados, aplicando o teorema de Leray-Hirsch (Teorema 4.2.7), obtemos:

$$
\begin{gathered}
\check{H}^{*}(B) \otimes \check{H}^{*}(\bar{Y}) \cong \check{H}^{*}(\bar{S}) \\
\sum_{i j} b_{i} \otimes i n c^{*}\left(u^{j}\right) \mapsto \sum_{i j} \bar{\pi}^{*}\left(b_{i}\right) \cup u^{j},
\end{gathered}
$$

no qual $i n c^{*}: \check{H}^{*}(\bar{S}) \longrightarrow \check{H}^{*}(\bar{Y})$ é induzida da inclusão $\bar{Y} \hookrightarrow \bar{S}$. Em outras palavras, $\check{H}^{*}(\bar{S})$ é um $\check{H}^{*}(B)$-módulo gerado por $1, u, \ldots, u^{m-1}$. Podemos expressar $u^{m}$ em termos da base:

$$
u^{m}+\omega_{1} u^{m-1}+\cdots+\omega_{m}=0
$$

no qual $\omega_{i} \in \check{H}^{i}(B)$.

Definição 5.2.5. Colocando $\omega_{0}=1$ e $\omega_{j}=0$, para $j \geq m+1$, definimos as classes de StiefelWhitney de $(\pi, \tau)$ como sendo os elementos $\omega_{i} \in \check{H}^{i}(B)$. O polinômio $\omega(t)=\sum_{j=0}^{m} \omega_{j} t^{m-j} e^{\prime}$ chamado de polinômio de Stiefel-Whitney de $(\pi, \tau)$.

Considere o homomorfismo entre $\check{H}^{*}(B)$-álgebras

$$
\begin{aligned}
\check{H}^{*}(B)[t] & \longrightarrow \check{H}^{*}(\bar{S}) \\
t & \mapsto u .
\end{aligned}
$$

Temos $\omega(t) \mapsto 0$. Seja $p(t)=a_{0}+a_{1} t+\cdots+a_{k} t^{k} \in \check{H}^{*}(B)[t]$ tal que $\left.p(t)\right|_{\bar{S}}=0$. Se $k<m$, como $\left\{1, u, \ldots, u^{k}\right\}$ é um conjunto linearmente independente em $\check{H}^{*}(\bar{S})$, temos $\bar{\pi}^{*}\left(a_{0}\right)=\ldots=$ $\bar{\pi}^{*}\left(a_{k}\right)=0$ e, portanto, $p(t) \equiv 0$ (polinômio nulo). Agora se $k \geq n$, como $\omega(t)$ tem 1 como coeficiente líder, existem $g(t), r(t) \in \check{H}^{*}(B)[t]$ (Vide [15, Capítulo 1, Proposição I.3.9, p. 24]) tais que:

$$
p(t)=g(t) \omega(t)+r(t)
$$

no qual o grau de $r(t)$ é menor do que $m$. Como $\left.p(t)\right|_{\bar{S}}=\left.\left.g(t)\right|_{\bar{S}} \omega(t)\right|_{\bar{S}}+\left.r(t)\right|_{\bar{S}}=\left.r(t)\right|_{\bar{S}}=0$ e $r(t)$ 
possui grau menor do que $m$, segue que $r(t) \equiv 0$ e, portanto, $p(t)=g(t) \omega(t)$. Assim, o núcleo do homomorfismo acima é gerado por $\omega(t)$ e, pelo Teorema do Isomorfismo, temos:

$$
\check{H}^{*}(\bar{S}) \cong \frac{\check{H}^{*}(B)[t]}{(\omega(t))}
$$

Teorema 5.2.6. Sejam $G=\mathbb{Z}_{2}, \pi: S \longrightarrow B$ um $G$-fibrado em esfera, $E^{\prime}$ um espaço com uma $G$ ação $\tau^{\prime}$ e uma aplicação $\pi^{\prime}:\left(E^{\prime}-Z^{\prime}\right) \longrightarrow B$, no qual $Z^{\prime}=F i x\left(\tau^{\prime}\right)$ (elementos que não mudam com a ação de $\left.\tau^{\prime}\right)$ tal que $\left(\pi^{\prime}, \tau^{\prime}\right)$ é um G-fibrado em esfera. Seja $f: S \longrightarrow E^{\prime}$ uma aplicação G-equivariante que preserva fibras em $S-Z$ (isto é, $\pi^{\prime} \circ f=\left.\pi\right|_{S-Z}$ ), no qual $Z=f^{-1}\left(Z^{\prime}\right)$. Agora, se $q(t) \in \check{H}^{*} B[t]$ é um polinômio tal que $\left.q(t)\right|_{\bar{Z}}=0$, então $q(t) \omega^{\prime}(t)=\omega(t) q^{\prime}(t)$, para algum polinômio $q^{\prime}(t) \in \check{H}^{*} B[t]$.

Demonstração: Como $\left.q(t)\right|_{\bar{Z}}=0$, pela continuidade da cohomologia de Čech (Vide Teorema 3.5.7, existe um aberto $V$ em $\bar{S}$ que contém $\bar{Z}$ e tal que $\left.q(t)\right|_{\bar{V}}=0$.

Pela exatidão da sequência $\check{H}^{*}(\bar{S}, V) \longrightarrow \check{H}^{*}(\bar{S}) \longrightarrow \check{H}^{*}(V)$, vemos que existe $v \in \check{H}^{*}(\bar{S}, V)$ tal que $j^{*}(v)=\left.q(t)\right|_{\bar{S}}$.

A função $f: S-Z \longrightarrow E^{\prime}-Z^{\prime}$ induz o homomorfismo $\bar{f}^{*}: \check{H}\left(\bar{E}^{\prime}-\bar{Z}^{\prime}\right) \longrightarrow \check{H}^{*}(\bar{S}-\bar{Z})$ tal que $\left.u^{\prime} \mapsto u\right|_{\bar{S}-\bar{Z}}$, no qual $u^{\prime},\left.u\right|_{\bar{S}-\bar{Z}}$ são classes características de Euler.

$\left.\operatorname{Temos} \omega^{\prime}(t)\right|_{\bar{S}-\bar{Z}}=\omega^{\prime}\left(\left.u\right|_{\bar{S}-\bar{Z}}\right)=\omega^{\prime}\left(\bar{f}^{*}\left(u^{\prime}\right)\right)=\bar{f}^{*}\left(\omega^{\prime}\left(u^{\prime}\right)\right)=0$.

De modo análogo ao argumento acima, existe $z \in \check{H}^{*}(\bar{S}, \bar{S}-\bar{Z})$ tal que $j^{*}(z)=\left.\omega^{\prime}(t)\right|_{\bar{S}}$.

O produto cup $v \cup z \in \check{H}^{*}(\bar{S}, V \cup(\bar{S}-\bar{Z}))=\check{H}^{*}(\bar{S}, \bar{S})=0$ é nulo. Consequentemente,

$$
\begin{aligned}
\left.q(t) \omega^{\prime}(t)\right|_{\bar{S}} & =\left.\left.q(t)\right|_{\bar{S}} \cup \omega^{\prime}(t)\right|_{\bar{S}} \\
& =j^{*}(v) \cup j^{*}(z) \quad \text { (naturalidade do produto cup) } \\
& =j^{*}(v \cup z) \\
& =0 .
\end{aligned}
$$

Como $\check{H}^{*}(\bar{S}) \cong \frac{\check{H}^{*}(B)[t]}{(\omega(t))}$, segue que $q(t) \omega^{\prime}(t)$ é um múltiplo de $\omega(t)$

OBS 5.2.7. O teorema acima generaliza o Teorema 5.1.2 De fato, sejam $\pi: E \longrightarrow B$, $\pi^{\prime}: E^{\prime} \longrightarrow B$ dois fibrados vetoriais. O fibrado associado em esfera $\pi: S(E) \longrightarrow B$ é um 
exemplo de um $\mathbb{Z}_{2}$-fibrado em esfera, no qual $\tau$ é a ação antipodal. Temos $Z^{\prime}=\{0\}$ e $Z=f^{-1}(0)$ e a função $f: S(E) \longrightarrow E^{\prime}$ do Teorema 5.1.2 satisfaz as hipóteses do Teorema 5.2.6

Exemplo 5.2.8. Seja $u: \xi^{n} \longrightarrow \xi^{\prime m}$ um B-morfismo com posto constante $k$. Considere a sequência exata $0 \longrightarrow K=\operatorname{ker}(u) \hookrightarrow \xi \stackrel{u}{\longrightarrow} I=\operatorname{Img}(u) \longrightarrow 0$. Então, $\xi \cong K \oplus I$. Assim $\omega(t)=\omega(K ; t) \omega(I ; t)$. Também, $0 \longrightarrow \operatorname{Img}(u) \hookrightarrow \eta \stackrel{\text { proj }}{\longrightarrow} K^{\prime}=$ Coker $(u) \longrightarrow 0$ é exata. Logo $\eta \cong I \oplus K^{\prime}$ e $\omega^{\prime}(t)=\omega(I ; t) \omega\left(K^{\prime} ; t\right)$. Consequentemente, $\omega(K ; t) \omega^{\prime}(t)=\omega(t) \omega\left(K^{\prime} ; t\right)$.

Note que $Z=S(E) \cap u^{-1}(0)=S(K)$ e $\left.\omega(K ; t)\right|_{\overline{S(K)}}=0$. Como $\check{H}^{*}(B)[t] \cong \frac{\check{H}^{*}(\overline{S K})}{(\omega(K ; t))}$, para $q(t) \in \check{H}^{*}(B)[t]$, segue que $\left.q(t)\right|_{\overline{S(K)}}=0$ se, e somente se, $q(t)$ é um múltiplo de $\omega(K ; t)$.

Então, se $\left.q(t)\right|_{\overline{S(K)}}=0$, existe $\lambda(t) \in \check{H}^{*}(B)[t]$ tal que $q(t)=\lambda(t) \omega(K ; t)$. Tomando $q^{\prime}(t)=$ $\lambda(t) \omega\left(K^{\prime} ; t\right)$, tem-se $q(t) \omega^{\prime}(t)=\omega(t) q^{\prime}(t)$. 


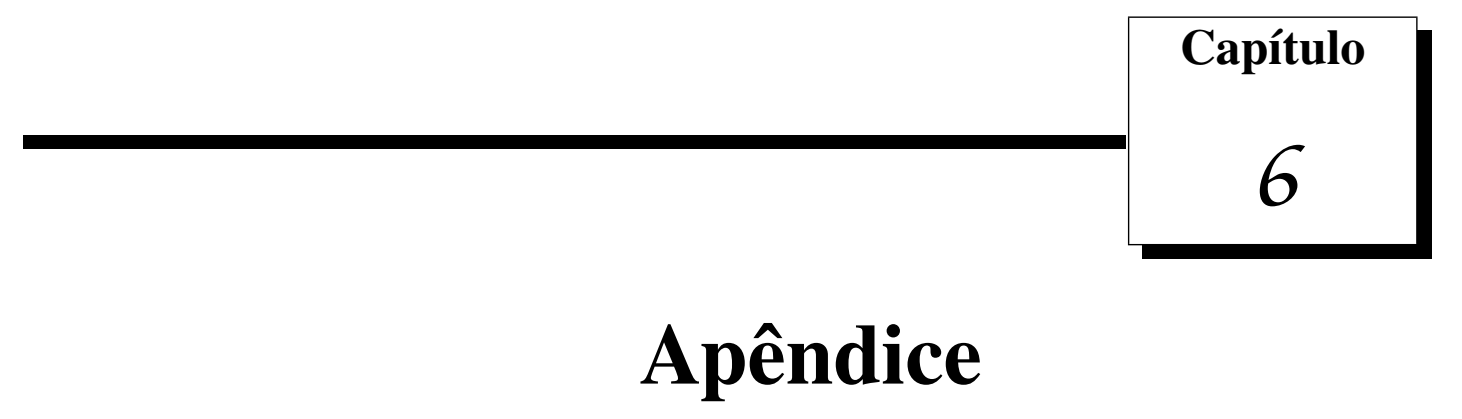

Esse capítulo tem o objetivo de definir alguns conceitos básicos encontrados no texto e apresentar resultados necessários para a complementação da leitura. Este capítulo, de forma alguma, preenche todas as necessidades para um texto autocontido. Muitos resultados são apenas enunciados, mas são dadas as devidas referências para suas demonstrações. As referências básicas para este capítulo são: Topology and Geometry de G. E. Bredon [3], Topology de J. Dugundji [12], Foundations of Algebraic Topology de S. Eilenberg e N. Steenrod [13], Characteristic Classes de J. W. Milnor e J. D. Stasheff [28], An Introduction to Homological Algebra de J. J. Rotman [32] e An Introduction to Homological Algebra de Weibel [39].

\subsection{Produto Tensorial}

Seja $R$ um anel comutativo com unidade.

Definição 6.1.1. Seja X um conjunto não vazio. Denotaremos por $R(X)$ o conjunto de todas as funções $f: X \longrightarrow R$ que são quase nulas, isto é, $f(x) \neq 0$ (elemento neutro da soma de R) apenas para um número finito de elementos $x \in X$. Fazendo $(f+g)(x)=f(x)+g(x) e$ $(r f)(x)=(r f(x))$, para $f, g \in R(X), r \in R$ e $x \in X$, o conjunto $R(X)$ é munido de uma estrutura de R-módulo. Os elementos $x \in X$ podem ser identificados com as funções $f_{x} \in R(X)$, que em $x$ valem 1(elemento neutro da multiplicação), e valem 0, caso contrário. Dessa forma, uma 
função $f$ pode ser representada como uma soma formal $\sum r_{i} x_{i}$. O R-módulo $R(X)$ é chamado $R$-módulo livre gerado por $X$.

Sejam $M, N$ dois $R$-módulos. Consideremos $R(M \times N)$ o $R$-módulo livre gerado pelos pares $(m, n) \in M \times N$ e $S$ o $R$-submódulo de $R(M \times N)$ gerado por elementos das seguintes formas:

$$
\begin{gathered}
\left(m+m^{\prime}, n\right)-(m, n)-\left(m^{\prime}, n\right), \\
\left(m, n+n^{\prime}\right)-(m, n)-\left(m, n^{\prime}\right), \\
(r m, n)-r(m, n), \\
(m, r n)-r(m, n),
\end{gathered}
$$

no qual $m, m^{\prime} \in M, n, n^{\prime} \in N$ e $r \in R$.

O produto tensorial de $M$ e $N$ é o $R$-módulo $M \otimes_{R} N=\frac{R(M \times N)}{S}$. Na maioria das vezes adotaremos a notação $\otimes$ ao invés de $\otimes_{R}$. As classes dos pares $(m, n)$ serão denotadas por $m \otimes n$. Estes elementos geram $M \otimes N$ e possuem as seguintes propriedades, para cada $m, m^{\prime} \in M$, $n, n^{\prime} \in N$ e $r \in R$.

- $\left(m+m^{\prime}\right) \otimes n=m \otimes n+m^{\prime} \otimes n$.

- $m \otimes\left(n+n^{\prime}\right)=m \otimes n+m \otimes n^{\prime}$

- $r m \otimes n=r(m \otimes n)=m \otimes r n$.

Tais propriedades seguem diretamente da definição.

Proposição 6.1.2. [13] Capítulo 5, Lema 9.2, p. 140]. Seja M um R-módulo, então $R \otimes M e$ $M \otimes R$ são isomorfos a $M$.

Demonstração: De fato, basta verificar que as seguintes aplicações $M \otimes R \rightarrow R, m \otimes r \mapsto m r$ e $R \otimes M \longrightarrow R, r \otimes m \mapsto r m$ são isomorfismos.

Definição 6.1.3. Sejam $f: M \longrightarrow M^{\prime}$ e $g: N \longrightarrow N^{\prime}$ dois R-homomorfismos. Então,

$$
\begin{aligned}
f \otimes g: M \otimes N & \longrightarrow M^{\prime} \otimes N^{\prime} \\
m \otimes n & \mapsto f(m) \otimes g(n)
\end{aligned}
$$


define um R-homomorfismo, chamado o homomorfismo induzido por $f$ e g.

Dado um $R$-módulo $A$, observemos que $\otimes A$ é funtor covariante entre a categoria $\operatorname{dos} R$ módulos. De fato, dado um $R$-homomorfismo $f: M \longrightarrow N$, obtemos $f \otimes 1_{A}: M \otimes A \longrightarrow N \otimes A$, definida por $m \otimes a \mapsto f(m) \otimes a$. Os fatos que comprovam que $\otimes A$ é um funtor covariante podem ser encontrados em [32, Capítulo 2, Teorema 2.48, p. 74]. Analogamente, $A \otimes$ é um funtor covariante.

Proposição 6.1.4. [32] Capítulo 2, Teorema 2.63, p. 84]. Seja A um R-módulo e consideremos a sequência exata curta

$$
0 \longrightarrow M \longrightarrow N \longrightarrow P \longrightarrow 0
$$

no qual $M, N$ e P são R-módulos. Então, $\otimes A$ e $A \otimes$ são funtores exatos à direitd! isto é,

$$
\begin{aligned}
& M \otimes A \longrightarrow N \otimes N \longrightarrow P \otimes A \longrightarrow 0 \\
& A \otimes M \longrightarrow A \otimes N \longrightarrow A \otimes P \longrightarrow 0
\end{aligned}
$$

são exatas. Se a sequência original cinde, então aplicando os funtores $\otimes A$ e $A \otimes$ obteremos sequências exatas:

$$
\begin{aligned}
& 0 \longrightarrow M \otimes A \longrightarrow N \otimes N \longrightarrow P \otimes A \longrightarrow 0 ; \\
& 0 \longrightarrow A \otimes M \longrightarrow A \otimes N \longrightarrow A \otimes P \longrightarrow 0 .
\end{aligned}
$$

\subsection{Sistemas Inversos}

Definição 6.2.1. Um sistema inverso de conjuntos $\{X, \pi\}$ sobre um conjunto dirigido $M$ é uma função que associa cada $\alpha \in M$ a um conjunto $X_{\alpha}$ e cada par $\alpha \leq \beta$ em $M$ a uma função $\pi_{\alpha}^{\beta}: X_{\beta} \longrightarrow X_{\alpha}$, no qual a família de funções $\pi_{\alpha}^{\beta}$ satisfazem as seguintes relações:

- $\pi_{\alpha}^{\alpha}=I d_{X_{\alpha}}$.

- Se $\alpha \leq \beta \leq \gamma$, então $\pi_{\alpha}^{\beta} \circ \pi_{\beta}^{\gamma}=\pi_{\alpha}^{\gamma}$.

\footnotetext{
${ }^{1}$ [32, Definição na página 86]
} 
As funções $\pi_{\alpha}^{\beta}$, assim como no caso do sistema direto, são chamadas projeções.

OBS 6.2.2. Um sistema inverso sobre um conjunto dirigido $M$ é um funtor contravariante.

Definição 6.2.3. Sejam $\{X, \pi\}$ e $\left\{X^{\prime}, \pi^{\prime}\right\}$ dois sistemas inversos sobre os conjuntos dirigidos $M e$ $M^{\prime}$, respectivamente. Uma aplicação entre os sistemas inversos $\{X, \pi\}$ e $\left\{X^{\prime}, \pi^{\prime}\right\}$, denotada por $\Phi:\{X, \pi\} \longrightarrow\left\{X^{\prime}, \pi^{\prime}\right\}$, consiste de uma aplicação entre conjuntos dirigidos $\phi: M^{\prime} \longrightarrow M$ e de funções $\phi_{\alpha^{\prime}}: X_{\phi\left(\alpha^{\prime}\right)} \longrightarrow X_{\alpha^{\prime}}^{\prime}$, com $\alpha^{\prime} \in M^{\prime}$, tais que se $\alpha^{\prime} \leq \beta^{\prime}$ em $M^{\prime}$, então o seguinte diagrama é comutativo.

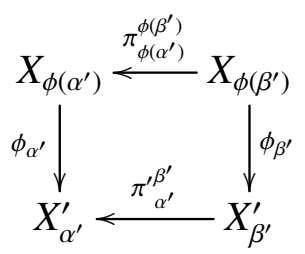

OBS 6.2.4. Como os sistemas diretos e suas aplicações, os sistemas inversos, juntamente com suas aplicações também formam uma categoria.

Definição 6.2.5. Seja $M^{\prime}$ um subconjunto dirigido do conjunto dirigido M. Dado um sistema inverso $\{X, \pi\}$ sobre $M$, os conjuntos e funções de $\{X, \pi\}$ que correspondem a conjuntos e relações indexadas em $M^{\prime}$, formam um sistema inverso $\left\{X^{\prime}, \pi^{\prime}\right\}$ sobre $M^{\prime}$, chamado subsistema inverso de $\{X, \pi\}$ sobre $M^{\prime}$. Se $M^{\prime}$ é cofinal em $M$, então o subsistema será chamado subsistema cofinal inverso de $\{X, \pi\}$ sobre $M^{\prime}$. A aplicação inclusão $\phi: M^{\prime} \longrightarrow M$ e as aplicações identidades $\phi_{\alpha}: X_{\alpha} \longrightarrow X_{\alpha}^{\prime}$, com $\alpha \in M^{\prime}$, formam uma aplicação $\Phi:\{X, \pi\} \longrightarrow\left\{X^{\prime}, \pi^{\prime}\right\}$ entre sistemas inversos.

\subsection{Limites Inversos}

Definição 6.3.1. Seja $\{X, \pi\}$ um sistema inverso de conjuntos sobre um conjunto dirigido $M . O$ limite inverso $X_{\infty}$ de $\{X, \pi\}$ é o subconjunto do produto $\prod_{\alpha \in M} X_{\alpha}$ consistindo de todos os elementos $x=\left(x_{\alpha}\right)_{\alpha \in M}$ tais que, para cada $\alpha \leq \beta \in M, \pi_{\alpha}^{\beta}\left(x_{\beta}\right)=x_{\alpha}$.

OBS 6.3.2. Se $\{X, \pi\}$ for um sistema inverso de espaços topológicos, então $X_{\infty}$ será um espaço topológico, com a topologia de subespaço de $\prod_{\alpha \in M} X_{\alpha}$. Para $R$-módulos, $X_{\infty}$ será um submódulo 
$d e \prod_{\alpha \in M} X_{\alpha}$.

Consideremos as projeções $\pi_{\alpha}: X_{\infty} \longrightarrow X_{\alpha}$ definidas por $\left(x_{\beta}\right)_{\beta \in M} \mapsto x_{\alpha}$.

Lema 6.3.3. [13. Capítulo 8, Lema 3.2, p. 215]. Sejam $\alpha \leq \beta$, então $\pi_{\alpha}=\pi_{\alpha}^{\beta} \pi_{\beta}$.

OBS 6.3.4. Se, no conjunto dirigido $M$, colocarmos um elemento $\infty$ tal que $\alpha \leq \infty$, para cada $\alpha \in M$ e considerarmos $M^{\prime}=M \cup\{\infty\}$, então o sistema inverso $\{X, \pi\}$ juntamente com $X_{\infty}$ e as projeções $\pi_{\alpha}$, formam um sistema inverso $\left\{X, \pi^{\prime}\right\}$ sobre $M^{\prime}$.

Definição 6.3.5. Seja $\Phi:\{X, \pi\} \longrightarrow\left\{X^{\prime}, \pi^{\prime}\right\}$ uma aplicação entre sistemas inversos. $O$ limite inverso de $\Phi$, denotado por $\Phi_{\infty}$, é uma aplicação

$$
\begin{aligned}
\Phi_{\infty}: \quad X_{\infty} & \longrightarrow X_{\infty}^{\prime} \\
\left(x_{\beta}\right)_{\beta \in M} & \mapsto\left(x_{\alpha}^{\prime}\right)_{\alpha \in M^{\prime}}=\left(\phi_{\alpha}\left(x_{\phi(\alpha)}\right)\right)_{\alpha \in M^{\prime}} .
\end{aligned}
$$

OBS 6.3.6. Segue da Definição 6.3.5 que, para cada $\alpha \in M^{\prime}$, o seguinte diagrama é comutativo.

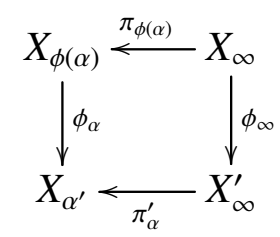

\subsection{Cohomologia de Alexander}

Sejam $X$ um espaço topológico e $G$ um $R$-módulo, no qual $R$ é um anel comutativo com unidade. O espaço produto $\underbrace{X \times \cdots \times X}_{(q+1)-\text { vezes }}$ será denotado por $X^{q+1}$. Para $q \geq 0, C^{q}(X ; G)$ denotará o $R$-módulo das funções $\varphi: X^{q+1} \longrightarrow G$. Muitas vezes omitiremos $G$ da notação anterior. A estrutura de $R$-módulo é dada por:

$$
\begin{aligned}
(r \varphi)\left(x_{0}, \ldots, x_{q}\right) & =r\left(\varphi\left(x_{0}, \ldots, x_{q}\right)\right) \\
\left(\varphi_{1}+\varphi_{2}\right)\left(x_{0}, \ldots, x_{q}\right) & =\varphi_{1}\left(x_{0}, \ldots, x_{q}\right)+\varphi_{2}\left(x_{0}, \ldots, x_{q}\right),
\end{aligned}
$$

no qual $r \in R, \varphi, \varphi_{1}, \varphi_{2} \in C^{q}(X ; G)$ e $\left(x_{0}, \ldots, x_{q}\right) \in X^{q+1}$. 
Um operador cobordo $\delta: C^{q}(X) \longrightarrow C^{q+1}(X)$ pode ser definido da seguinte forma:

$$
(\delta \varphi)\left(x_{0}, \ldots, x_{q+1}\right) \mapsto \sum_{0 \leq 1 \leq q+1}(-1)^{i} \varphi\left(x_{0}, \ldots, \hat{x}_{i}, \ldots, x_{q+1}\right)
$$

Então, $\delta \delta=0$ e $C^{*}(X)=\left\{C^{q}(X), \delta\right\}$ é um complexo de cocadeias sobre $R$.

Definição 6.4.1. Um elemento $\varphi \in C^{q}(X)$ é localmente nulo se existe uma cobertura $\left\{U_{\alpha}\right\}$ de $X$, por conjuntos abertos, tal que $\varphi$ se anula em $U_{\alpha}^{q+1}$, para todo $\alpha$. Neste caso, note que $\delta(\varphi)$ se anula em $U_{\alpha}^{q+2}$, para todo $\alpha$. O conjunto de todas as funções $\varphi \in C^{q}(X)$ localmente nulas formam um submódulo denotado por $C_{0}^{q}(X)$. Então, $C_{0}^{*}(X)=\left\{C_{0}^{q}(X), \delta\right\}$ é um subcomplexo de cocadeias de $C^{*}(X)$. Denotemos por $\bar{C}^{*}(X)$ o complexo de cocadeias dado pelo quociente de $C^{*}(X)$ por $C_{0}^{*}(X)$. O módulo de cohomologia de $\bar{C}^{*}(X)$ de grau q com coeficientes em $G$ será denotado por $\bar{H}^{q}(X ; G)$.

Dada uma função contínua $f: X \longrightarrow Y$, existe uma aplicação de cocadeias induzida $f^{\#}$ : $C^{*}(Y) \longrightarrow C^{*}(X)$ definida por $f^{\#}(\varphi)\left(x_{0}, \ldots, x_{q}\right)=\varphi\left(f\left(x_{0}\right), \ldots, f\left(x_{q}\right)\right)$, no qual $\varphi \in C^{q}(Y)$ e $x_{0}, \ldots, x_{q} \in X$. Como $f^{\#}$ leva $C_{0}^{*}(Y)$ em $C_{0}^{*}(X)$, então existe uma aplicação de cocadeias induzida $f^{\#}: \bar{C}^{*}(Y) \longrightarrow \bar{C}^{*}(X)$. Se $A \subset X$ e $i: A \hookrightarrow X$ é a inclusão, o núcleo de $i^{\#}: \bar{C}^{*}(X) \longrightarrow \bar{C}^{*}(A)$ é um subcomplexo de $\bar{C}^{*}(X)$ e será denotado por $\bar{C}^{*}(X, A)$.

A construção anterior define uma teoria de cohomologia, chamada teoria de cohomologia de Alexander [33, Capítulo 6, p.306].

\subsubsection{Sequência de Mayer-Vietoris e o produto cup}

Sejam $X_{1}$ e $X_{2}$ conjuntos abertos em $X_{1} \cup X_{2}$. Consideremos as inclusões $i_{1}: X_{1} \hookrightarrow X_{1} \cup X_{2}$, $i_{2}: X_{2} \hookrightarrow X_{1} \cup X_{2}, j_{1}: X_{1} \cap X_{2} \hookrightarrow X_{1}$ e $j_{2}: X_{1} \cap X_{2} \hookrightarrow X_{2}$

Teorema 6.4.2. A seguinte sequência é exata.

$$
0 \longrightarrow \bar{C}^{q}\left(X_{1} \cup X_{2}\right) \stackrel{\left(i_{1}^{\#}, i_{2}^{*}\right)}{\longrightarrow} \bar{C}^{q}\left(X_{1}\right) \oplus \bar{C}^{q}\left(X_{2}\right) \stackrel{\left(j_{1}^{\#}-j_{2}^{*}\right)}{\longrightarrow} \bar{C}^{q}\left(X_{1} \cap X_{2}\right) \longrightarrow 0 .
$$

\section{Demonstração:}

1. $\left(i_{1}^{\#}, i_{2}^{\#}\right): \bar{C}^{q}\left(X_{1} \cup X_{2}\right) \longrightarrow \bar{C}^{q}\left(X_{1}\right) \oplus \bar{C}^{q}\left(X_{2}\right)$ é injetora. 
Seja $\bar{\varphi} \in \bar{C}^{q}\left(X_{1} \cup X_{2}\right)$ tal que $\left(i_{1}^{\#}(\bar{\varphi}), i_{2}^{\#}(\bar{\varphi})\right)=(0,0)$. Então, existem coberturas abertas $\left\{\left(U_{1}\right)_{\alpha}\right\},\left\{\left(U_{2}\right)_{\beta}\right\}$ de $X_{1}, X_{2}$ tais que $\left.\varphi\right|_{X_{1}^{q}}$ e $\left.\varphi\right|_{X_{2}^{q}}$ se anulam, respectivamente. Assim, $\varphi$ se anula em $\left\{\left(U_{1}\right)_{\alpha},\left(U_{2}\right)_{\beta}\right\}$. Logo, $\bar{\varphi}=0$.

2. $\operatorname{Img}\left(\left(i_{1}^{\#}, i_{2}^{\#}\right)\right) \subset \operatorname{ker}\left(j_{1}^{\#}-j_{2}^{\#}\right)$.

Esta inclusão é imediata.

3. $\operatorname{ker}\left(j_{1}^{\#}-j_{2}^{\#}\right) \subset \operatorname{Img}\left(\left(i_{1}^{\#}, i_{2}^{\#}\right)\right)$.

Seja $\left(j_{1}^{\#}-j_{2}^{\#}\right)\left(\overline{\varphi_{1}}, \overline{\varphi_{2}}\right)=(0,0)$. Então, $\left.\varphi_{1}\right|_{\left(X_{1} \cap X_{2}\right)^{q+1}}=\left.\varphi_{2}\right|_{\left(X_{1} \cap X_{2}\right)^{q+1}}$. Tome $\varphi:\left(X_{1} \cup X_{2}\right)^{q+1} \longrightarrow$ $G$, no qual $\left.\varphi\right|_{X_{1}^{q+1}}=\varphi_{1},\left.\varphi\right|_{X_{2}^{q+1}}=\varphi_{2}$ e $\varphi=e$, caso contrário. Então, $\left(i_{1}^{\#}, i_{2}^{\#}\right) \bar{\varphi}=\left(\overline{\varphi_{1}}, \overline{\varphi_{2}}\right)$.

4. $\left(j_{1}^{\#}-j_{2}^{\#}\right)$ é sobrejetora.

Dada $\overline{\varphi^{\prime}} \in \bar{C}^{q}\left(X_{1} \cap X_{2}\right)$, tome $\varphi_{1} \in C^{q}\left(X_{1}\right), \varphi_{2} \in C^{q}\left(X_{2}\right)$, no qual $\left.\varphi_{1}\right|_{\left(X_{1} \cap X_{2}\right)^{q+1}}=\varphi^{\prime}$ e $\varphi_{1}=e$, caso contrário, e $\varphi_{2}=e$. Então, $\left(j_{1}^{\#}-j_{2}^{\#}\right)\left(\overline{\varphi_{1}}, \overline{\varphi_{2}}\right)=\overline{\varphi^{\prime}}$.

Segue do Teorema 6.4.2, que existe uma sequência exata longa [39, Capítulo 1, Teorema 1.3.1, p. 11]

$$
\cdots \longrightarrow \bar{H}^{q}\left(X_{1} \cup X_{2}\right) \stackrel{\left(i_{1}^{*}, i^{*}\right)}{\longrightarrow} \bar{H}^{q}\left(X_{1}\right) \oplus \bar{H}^{q}\left(X_{2}\right) \stackrel{\left(j_{1}^{*}-j_{2}^{*}\right)}{\longrightarrow} \bar{H}^{q}\left(X_{1} \cap X_{2}\right) \stackrel{\delta}{\longrightarrow} \bar{H}^{q+1}\left(X_{1} \cup X_{2}\right) \longrightarrow \cdots
$$

chamada sequência de Mayer-Vietoris, no qual $\delta$ é um homomorfismo conectante .

O produto cup para a teoria de cohomologia de Alexander pode ser obtido como segue [33, Capítulo 6, p. 315]. Sejam $X_{1}$ e $X_{2}$ subespaços de $X$, no qual um elemento de $C^{p+q}(X ; G)$ que é localmente nulo em $C^{p}\left(X_{1} ; G\right)$ e em $C^{q}\left(X_{2} ; G\right)$ é localmente nulo em $C^{p+q}\left(X, X_{1} \cup X_{2}\right)$. Isto acontece quando $X_{1}$ e $X_{2}$ são abertos em $X_{1} \cup X_{2}$. Então, temos:

$$
\cup: \bar{H}^{p}\left(X, X_{1} ; G\right) \otimes \bar{H}^{q}\left(X, X_{2} ; G\right) \longrightarrow \bar{H}^{p+q}\left(X, X_{1} \cup X_{2} ; G\right)
$$

As seguintes propriedades são verificadas:

1. Naturalidade. Seja $f: X \longrightarrow Y$ uma função contínua que leva $X_{1}\left(X_{2}\right)$ em $Y_{1}\left(Y_{2}\right)$. Sejam $u \in \bar{H}^{p}\left(Y, Y_{1} ; G\right)$ e $v \in \bar{H}^{q}\left(Y, Y_{2} ; G\right)$ e sejam $f_{1}:\left(X, X_{1}\right) \longrightarrow\left(Y, X_{1}\right), f_{2}:\left(X, X_{2}\right) \longrightarrow\left(Y, Y_{2}\right)$ e $\bar{f}:\left(X, X_{1} \cup X_{2}\right) \longrightarrow\left(Y, Y_{1} \cup Y_{2}\right)$. Então: 


$$
\bar{f}^{*}(u \cup v)=f_{1}^{*}(u) \cup f_{2}^{*}(v) .
$$

2. Associatividade. Se $X_{1}, X_{2}, X_{3}$ são abertos em $X$ e $u \in \bar{H}^{p}\left(X, X_{1} ; G\right), v \in \bar{H}^{q}\left(X, X_{2} ; G\right)$ e $z \in \bar{H}^{r}\left(X, X_{3} ; G\right)$, então:

$$
(u \cup v) \cup z=u \cup(v \cup z) .
$$

3. Anticomutatividade. Se $X_{1}, X_{2}$ são abertos em $X$ e $u \in \bar{H}^{p}\left(X, X_{1} ; G\right), v \in \bar{H}^{q}\left(X, X_{2} ; G\right)$, então:

$$
u \cup v=(-1)^{p q}(v \cup u)
$$

\subsection{Complexo CW}

Definição 6.5.1. Em $\mathbb{R}^{n}$, o disco unitário será denotado por $D^{n}$. Qualquer espaço homeomorfo a $D^{n}$ é chamado uma n-célula fechada $e$, qualquer espaço homeomorfo ao interior de $D^{n}$,é chamado uma n-célula aberta.

Definição 6.5.2. Um complexo $\boldsymbol{C W}$ consiste de um espaço de Hausdorff X e uma coleção $\left\{e_{\alpha}\right\}$ de subconjuntos de X, que satisfazem:

(1) Cada $e_{\alpha}$ é uma $n(\alpha)$-célula aberta, no qual $n(\alpha) \geq 0$. Para cada célula $e_{\alpha}$, existe uma função contínua $f: D^{n(\alpha)} \longrightarrow X$, que leva o interior de $D^{n(\alpha)}$ homeomorficamente em $e_{\alpha}$. A função $f$ é chamada função caractenística para a célula $e_{\alpha}$.

(2) Se $x \in \overline{e_{\alpha}}-e_{\alpha}$, então $x \in e_{\beta}$, no qual $n(\beta)<n(\alpha)$.

Um subcomplexo de $X$ é um subconjunto fechado A (na topologia de X) que é uma reunião de células $e_{\alpha}$. Caso a reunião seja finita, A é chamado um subcomplexo finito.

(3) Cada ponto de X está contido em algum subcomplexo finito.

(4) [Topologia de Whitehead] Um subconjunto A de X é fechado se, e somente se, a interseção de A com cada subcomplexo finito for fechado.

OBS 6.5.3. Observemos que se X possui um número finito de células $e_{\alpha}$, então as duas últimas condições já estarão imediatamente satisfeitas. 
Exemplos 6.5.4. (i) Seja $X=S^{n}$, a esfera unitária. O pólo norte da esfera é uma 0-célula aberta e a projeção estereográfica é um homeomorfismo de $S^{n}-(0, \ldots, 0,1)$ com $\mathbb{R}^{n}$. Portanto, $S^{n}$ possui uma estrutura de complexo $C W$ finito, formado por uma 0-célula e uma n-célula abertas.

(ii) Os espaços $\mathbb{R}^{n}$ e $\mathbb{R} P^{n}$ também são exemplos de complexos $C W$ finitos [18, Capítulo 0, p. 6].

OBS 6.5.5. Note que o fecho $\overline{e_{\alpha}}$ de uma célula de X não é necessariamente uma célula, como mostra o Exemplo 6.5.4(i).

\subsection{Paracompacidade}

Uma outra maneira de definirmos cobertura de um espaço topológico é dada a seguir.

Definição 6.6.1. Uma coleção $\left\{U_{\alpha}\right\}$ de subconjuntos de um espaço topológico $X$ é uma cober-

tura de $X$, se $X=\bigcup_{\alpha} U_{\alpha}$. Se cada $U_{\alpha}$ for um subconjunto aberto de $X$, dizemos que a cobertura $\left\{U_{\alpha}\right\}$ é aberta. Um refinamento para uma cobertura $\left\{U_{\alpha}\right\}$ de $X$ é uma coleção $\left\{V_{\beta}\right\}$ tal que cada $V_{\beta}$ é um subconjunto de algum $U_{\alpha}$.

Definição 6.6.2. Sejam X um espaço topológico $e\left\{U_{\alpha}\right\}$ uma cobertura aberta de $X$. Dizemos que $\left\{U_{\alpha}\right\}$ é localmente finita se, para cada $x \in X$, existe uma vizinhança aberta $V$ de $x$ tal que $U_{\alpha} \cap V \neq \emptyset$, apenas para um número finito de índices $\alpha$.

Definição 6.6.3. Um espaço topológico de Hausdorff X é paracompacto se, para cada cobertura aberta $\left\{U_{\alpha}\right\}$ de $X$, existir um refinamento de $\left\{U_{\alpha}\right\}$ localmente finito.

Proposição 6.6.4. [17] Capítulo 1, Proposição 1.18, p. 35]. Todo espaço compacto de Hausdorffé paracompacto.

Outros resultados são dados na seguinte proposição.

Proposição 6.6.5. [3] Capítulo 1, Proposições 12.4 e 12.5, p. 36]. Um subconjunto fechado de um espaço paracompacto é paracompacto. Todo espaço paracompacto é normal.

Mas, uma das mais importantes propriedades de um espaço paracompacto é o fato deste possuir uma partição da unidade subordinada a toda família de abertos que o cobre. 
Definição 6.6.6. Seja $\left\{U_{\alpha}\right\}$ uma cobertura aberta de um espaço topológico X. Uma partição da unidade subordinada a esta cobertura é uma coleção de funções contínuas $\left\{\phi_{\beta}: X \longrightarrow[0,1]\right\}$ satisfazendo as seguintes condições:

(1) Existe um refinamento localmente finito $\left\{V_{\beta}\right\}$ de $\left\{U_{\alpha}\right\}$, no qual $\overline{\phi_{\beta}^{-1}((0,1])} \subset V_{\beta}$ (suporte de $\left.\phi_{\beta}\right)$.

(2) $\sum_{\beta} \phi_{\beta}(x)=1$, para todo $x \in X$.

Teorema 6.6.7. [3] Capítulo 1, Teorema 12.8, p. 37]. Seja X um espaço paracompacto e $\left\{U_{\alpha}\right\}$ uma cobertura aberta de X. Então, existe uma partição da unidade subordinada a $\left\{U_{\alpha}\right\}$.

Uma segunda definição para espaços paracompactos é dada a seguir.

Definição 6.6.8. [17. Capítulo 1, p. 35]. Um espaço topológico de HausdorffX é paracompacto se, para cada cobertura aberta $\left\{U_{\alpha}\right\}$ de $X$, existir uma partição da unidade $\left\{\phi_{\beta}\right\}$ subordinada a esta cobertura.

OBS 6.6.9. As Definições 6.6.3 e.6.8 são equivalentes. De fato, para verificar que a segunda definição implica na primeira, basta tomarmos $\left\{\phi_{\beta}^{-1}(0,1]\right\}$ como sendo a cobertura localmente finita procurada. A recíproca é dada pelo Teorema 6.6.7.

Proposição 6.6.10. [17. Capítulo 1, Proposição 1.20, p. 36]. Todo complexo CW é paracompacto. 


\section{Referências Bibliográficas}

[1] Borsuk, K. Drei Sätze über die n-dimensionale euklidische Sphäre, Fund. Math. 20 177190, (1933).

[2] Bredon, G., Introduction to Compact Transformation Groups, Academic Press, INC., New York and London (1972).

[3] Bredon, G.E., Topology and Geometry, Graduate Texts in Math. 139 Springer-Verlag New York Inc., (1993).

[4] Capel, C. E., Inverse Limit Spaces, J. vol. 21 pp. 233-246 (1954).

[5] De Mattos, D. and Dos Santos, E.L. A parametrized Borsuk-Ulam theorem for a product of spheres with free $\mathbb{Z}_{p}$-action and free $S^{1}$-action. Algebraic and Geometric Topology 7 1791-1804, (2007).

[6] Dieck, T., Transformation Groups, Walter de Gruyter, Berlin-New York, (1987).

[7] Dieck, T., Algebraic Topology, EMS Textbooks in Mathematics, European Mathematical Society, 2008.

[8] Dold, A. Lectures on Algebraic Topology, Die Grundlehren der mathematischen Wissenschaften, Band 200, Springer - Verlag, (1972).

[9] Dold, A., Parametrized Borsuk-Ulam theorems, Comment. Math. Helv., 63 (1988) 275285. 
[10] Dowker, C. H., Čech Cohomology Theory and the Axioms, The Annals of Mathematics, Vol. 51, no. 2, (1950).

[11] Dowker, C. H., Homology Groups of Relations, The Annals of Mathematics, Second Series, Vol. 56, n. 1, pp. 84-95, (Jul. 1952).

[12] Dugundji, J., Topology, Allyn and Bacon, Inc.,Boston, (1966).

[13] Eilenberg, S. and Steenrod, N., Foundations of Algebraic Topology, Princeton Mathematical Series n.15, Princeton University Press, (1952).

[14] Engelking, R., Dimension Theory, North-Holland Publishing Company, (1978).

[15] Garcia, A. e Lequain, Y., Elementos de Álgebra 3 ed., Projeto Euclides, Rio de Janeiro, IMPA, (2005).

[16] Greenberg, M. J. and Harper J.R. Algebraic Topology, a first course. Addison-Wesley Publishing Company, Inc. New York Amsterdam, (1981).

[17] Hatcher, A. Vector Bundles and K-theory, Version 1.1, November (2000).

[18] Hatcher, A. Algebraic Topology, Cambridge University Press (2002).

[19] Husemoller, D., Fiber Bundles, Second Edition, Springer-Verlag, New York (1975).

[20] Hurewicz, W., Dugundji, J. and Dowker C. H., Continuous Connectivity Groups in Terms of Limit Groups, The Annals of Mathematics, Second Series, Vol. 49. n. 2, pp. 391-406, (Apr. 1948).

[21] Jaworowski, J., Bundles with periodic maps and mod $p$ Chern polynomial, Proc. of the Amer. Math. Soc. 132, no. 4, (2004) 1223-1228.

[22] Izydorek, M. and Rybicki, S., On parametrized Borsuk-Ulam theorem for free $\mathbb{Z}_{p}$-action, Barcelona Conference on Algebraic Topology, (1990) 227-234.

[23] Keesee, J. W. On the homotopy axiom, Annal of Mathematics Vol. 54 n. 2 (Sep. 1951). 
[24] Koikara, B.S. and Mukerjee, H.K., A Borsuk-Ulam theorem type for a product of spheres, Topology and its Applications, 63 (1995) 39-52.

[25] Lima, E.L., Variedades Diferenciáveis, Publicações Matemáticas, Impa, (2008).

[26] Lima, E.L., Homologia Básica 1 ed., Porjeto Euclides, Rio de Janeiro, IMPA, (2009).

[27] Massey, W. S., How to give an exposition of the Cech-Alexander-Spanier type homology theory, The American Mathematical Monthly, Vol. 85, n. 2 , pp. 75-83, (Feb. 1978).

[28] Milnor, J. W. and Stasheff, J. D., Characteristic Classes, Annals of Mathematics Studies n. 76, Princeton Univ. Press, Princeton, New Jersey and Univ. of Tokyo Press, (1974).

[29] Munkres, J.R., Elements of Algebraic Topology, The Benjamin/Cummings Publishing Company, Inc., Cambridge, Massachusetts, (1984).

[30] Munkres, J.R., Topology, A First Course, Prentice Hall, (2000).

[31] Nakaoka, M., Parametrized Borsuk-Ulam theorems and characteristic polynomials. Proceedings of the Tianjin Fixed Point Conference 1988, Lectures Notes in Math., Springer Verlag, 1411, (1989).

[32] Rotman, J. J., An Introduction to Homological Algebra, Second Edition, Springer-Verlag, Universitex, (2008).

[33] Spanier, E.H., Algebraic Topology, Springer, Berlin, (1966).

[34] Spanier, E. H., Cohomology Theory for General Spaces, The Annals of Mathematics, Second Series, Vol. 49, n. 2, pp. 407-427, (Apr. 1948).

[35] Steenrod, N., The topology of fiber bundles. Princeton Mathematical Series n. 14, Princeton University Press, (1951).

[36] Volovikov, A. Y., On fibre-preserving G-maps. Uspekhi Mat. Nauk , 51, no. 3, (1996) 189-190(Russian); translation in Russian Math. Surveys, 51, no. 3, (1996), 575-577.

[37] Yang, C.T., On theorems of Borsuk-Ulam, Kakutani-Yamabe-Yujobo and Dyson. I, Ann. of Math. 60:2, 262-282 (1954). 
[38] Wallace, A.H., Algebraic Topology, Homology and Cohomology W. A. Benjamin Inc., (1970).

[39] Weibel, C. A., An Introduction to Homological Algebra, Cambridge, University Press (1994). 


\section{Índice Remissivo}

Ação

à direita, 18

à esquerda, 18

efetiva, 19

livre, 19

trivial, 19

Aplicação

$G$-equivariante, 18

de Gauss, 8

de pares simpliciais, 33

entre conjuntos dirigidos, 28

entre sistemas diretos, 28

entre sistemas inversos, 72

fatoração, 55

simplicial, 32

simplicial contígua, 33

Atlas

de um fibrado, 25

de um fibrado vetorial, 2

Axioma(s)

da Continuidade, 40

de Eilenberg-Steenrod, 39

dimensão, 40
B-isomorfismo

entre $G$-fibrados triviais, 21

entre fibrados, 24

entre fibrados vetoriais, 4

B-morfismo

entre $G$-fibrados principais, 21

entre fibrados, 24

entre fibrados vetoriais, 3

Carta local

de um fibrado, 25

de um fibrado vetorial, 2

Categoria

VB, 3

dos $R$-módulos, 39

dos pares simpliciais, 33

dos pares topológicos, 39

$\mathrm{P}, 13$

$\mathrm{PB}, 21$

SET, 13

Classe

característica de Euler, 57

de Stiefel-Whitney, 50 
de Stiefel-Whitney de $(\pi, \tau), 65$

de Thom, 56

Cobertura aberta, 36, 77

Cohomologia

de Čech, 38

de Alexander, 73

simplicial, 31

Complemento ortogonal

de um subfibrado vetorial, 11

Complexo

CW, 76

de cocadeias, 35

simplicial abstrato, 32

Condição de trivialidade local, 2

Conjunto dirigido, 27

Dimensão

axioma, 40

cohomológica, 61

de um simplexo, 32

topológica, 63

Espaço

base

de um $G$-fibrado principal, 20

de um fibrado vetorial, 2

paracompacto, 77

total

de um $G$-fibrado principal, 20

de um fibrado vetorial, 2

Exatidão, 40
Excisão, 40

Extensão de cohomologia da fibra, 49

Face, 32

Fibra

de um $G$-fibrado principal, 20

de um fibrado vetorial, 2

Fibrado

associado a um $G$-fibrado principal, 23

coordenado, 25

em esfera associado, 12

localmente isomorfos, 24

localmente trivial, 24

projetivo associado, 13

trivial, 24

Fibrado vetorial

induzido, 7

canônico, 5

complemento ortogonal, 11

em linha, 6

em linha canônico, 6

métrica, 10

normal, 3

real, 1

restrição, 8

tangente, 2

trivial, 2

universal, 14

Função

característica, 76

translação, 19 
G-espaço

à direita, 18

G-fibrado

em esfera, 63

principal, 20

principal induzido, 22

principal localmente isomorfos, 22

principal localmente trivial, 22

principal trivial, 20

Grupo estrutural, 23

Grupo topológico, 17

Homomorfismo induzido, 39

Injeção de um subsistema, 29

Invariância homotópica, 39

Isomorfismo

entre $G$-fibrados principais, 21

k-referencial, 4

Limite

direto, 29

direto de uma aplicação, 31

inverso, 72

inverso de uma aplicação, 73

Localmente

finita, 77

nulo, 74

\section{Métrica}

de um fibrado vetorial, 10

Mapping cylinder, 57
Morfismo

entre fibrados, 24

B-isomofismo

entre fibrados vetoriais, 4

B-isomorfismo

entre fibrados, 24

B-morfismo

entre $G$-fibrados principais, 21

entre fibrados, 24

entre fibrados vetoriais, 3

entre $G$-fibrados principais, 21

entre fibrados vetoriais, 3

Isomorfismo

entre $G$-fibrados principais, 21

n-célula

aberta, 76

fechada, 76

Nervo, 36

Operador

bordo, 34

cobordo relativo, 35

Órbita, 19

Orientação, 33

Par fibrado, 49

em disco, 49

Par simplicial, 32

Partição da unidade, 78

Polinômio

de Stiefel-Whitney, 60 
de Stiefel-Whitney de $(\pi, \tau), 65$

Produto

cross $\times, 45$

cup $\cup, 44$

tensorial $\otimes, 70$

Produto de Whitney, 50

Projeção

de um $G$-fibrado principal, 20

de um fibrado vetorial, 2

de um sistema inverso, 72

de uma sistema direto, 28

entre coberturas, 37

q-ésimo $R$-módulo

de cohomologia de Čech, 38

q-dimensional esqueleto, 32

q-funtor cohomologia Čech, 39

q-simplexo, 32

q-simplexo orientado, 33

R-módulo

de $q$-cadeias simpliciais, 34

de $q$-cadeias simpliciais relativo, 35

de $q$-cocadeias simpliciais relativo, 35

de cohomologia orientado, 35

livre, 70

Refinamento, 36, 77

Relação, 29

Restrição

de um fibrado vetorial, 8

Sequência de Gysin, 57

de Mayer-Vietoris, 75

exata curta, 16

Simplexos, 32

Sistema

de funções transições, 25

direto, 28

inverso, 71

Soma de Whitney, 7

Subcomplexo, 32

CW, 76

Subconjunto

dirigido, 27

dirigido cofinal, 28

Subfibrado vetorial, 2

Subsistema

cofinal direto, 29

cofinal inverso, 72

direto, 28

inverso, 72

Teorema

Borsuk-Ulam

Clássico, 62

versão parametrizada, 59

do isomorfismo de Thom, 56

Leray-Hirsch, 48

Redução, 41

Vértices, 32

Variedade 
de Grassmann, 5

de Stiefel, 4 